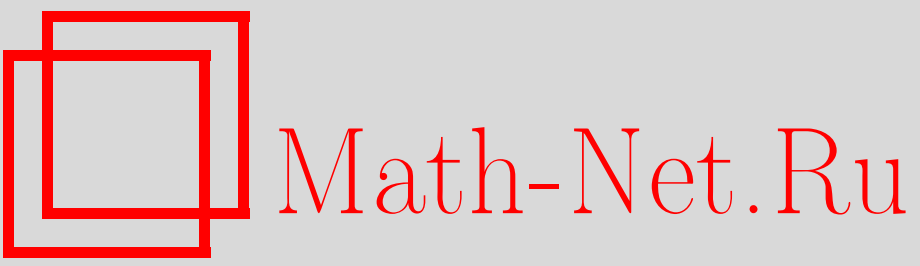

М. В. Ружанский, Особенности аффинных слоений в теории регулярности интегральных операторов Фурье, УМН, 2000, том 55, выпуск 1, 99-170

DOI: https://doi.org/10.4213/rm250

Использование Общероссийского математического портала Math-Net.Ru подразумевает, что вы прочитали и согласны с пользовательским соглашением

http://www.mathnet.ru/rus/agreement

Параметры загрузки:

IP: 34.227 .88 .159

26 апреля 2023 г., 15:36:10 


\title{
ОСОБЕННОСТИ АФФИННЫХ СЛОЕНИЙ В ТЕОРИИ РЕГУЛЯРНОСТИ ИНТЕГРАЛЬНЫХ ОПЕРАТОРОВ ФУРЬЕ
}

\author{
М.В. РужАнСКИЙ
}

В статье рассматриваются свойства непрерывности интегральных операторов Фурье в различных функциональных пространствах. Наибольший интерес вызывают пространства $L_{p}$, для которых проведен обзор последних резултатов. Так, точные поря дки известны для операторов, удовлетворяющих так называемому условию гладкой факторизации. Далее в статье это условие исследуется в вещественной и комплексной постановках. В последнем случае условия на непрерывность интегральных операторов Фурье связаны с особенностями аффинных слоений в (подмножествах) $\mathbb{C}^{n}$, задаваемых ядрами матриц Якоби голоморфных отображений. Проведен анализ особенностей таких слоений в общем случае. В частности, показано, что при неболшших размерностях $n$ или при небольших рангах матрищы Якоби все особенности слоений устранимы. Случай интегральных операторов Фурье приводит к слоениям, задаваемым ядрами Гессиана фазовой функции оператора. Опираясь на проведенный анализ особенностей для операторов, коммутирующих со сдвигами, в ряде случаев показано, что условие гладкой факторизации автоматически выполняется, из чего следуют $L^{p}$ оценки. В остальных случаях приведены примеры нарушения условия гладкой факторизации. Результаты применены к $L^{p}$ оценкам решений задачи Коши для гиперболических уравнений в частных производных.

Библиография: 68 названий.

\section{СОДЕРЖАНИЕ}

1. Введение

1.1. Регулярность интегральных операторов Фурье . . . . . . . . . . . . . . . 100

1.2. Слоения с афффинными слоями ............................... 106

1.3. Формулировка проблемы для параметризованных слоений ........... 108

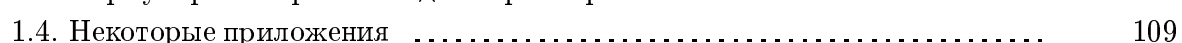

2. Оценки для интегральных операторов Фурье ........................ 112

2.1. Интегралњные операторы Фурье .............................. 112

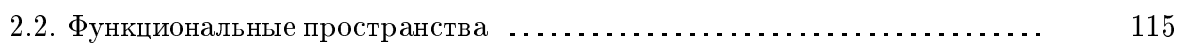

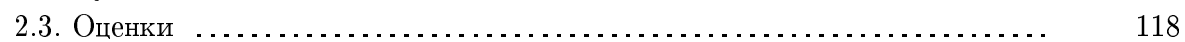

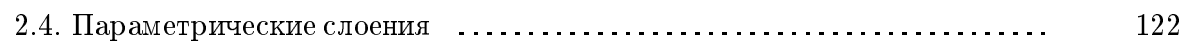

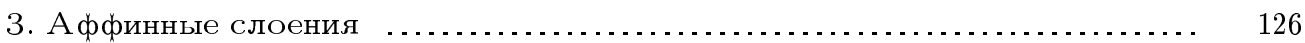

3.1. Методы комплексной аналитической геометрии . .................. 126

3.2. Основные результаты .................................... 128

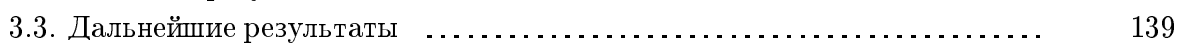


4. Аффинные слоения градиентного типа ............................... 141

4.1. Локализация ............................................. 141

4.2. Слоения градиентного типа ................................. 144

4.3. Восстановление фазовой функции ............................ 148

4.4. Существование сингулярных слоений градиентного типа ............. 153

5. Дальнейшие оценки для интегральных операторов Фурье . . . . . . . . . 155

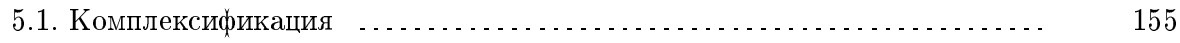

5.2. Операторы, коммутирующие со сдвигами ...................... 156

5.3. Применение к интегральным операторам Фурье . . . . . . . . . . . . . . . . . . . 157

6. Задача Коши для гиперболических уравнений …................ 160

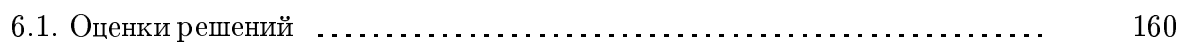

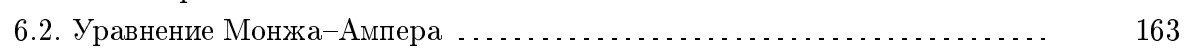

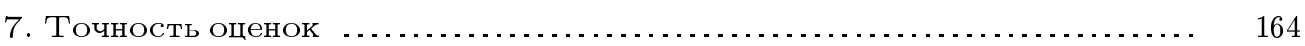

7.1. Существенно однородный случай … . . . . . . . . . . . . . . . . . . . . . . 164

7.2. Представление непрерывных операторов небольших отрицательных по-

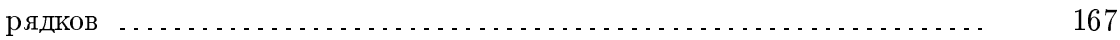

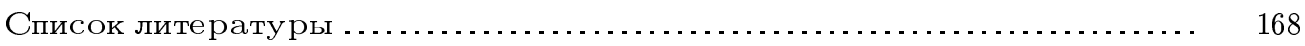

\section{1. Введение}

1.1. Регулярность интегральных операторов Фурье. Теория регулярности решений уравнений в частных производных интересует математиков в течение длительного времени. Одним из важных примеров является волновое уравнение, решение которого хорошо изучено и допускает представление в виде суммы двух эллиптических интегральных операторов Фурье с фазовыми функциями, решающими уравнение эйконала геометрической оптики. В общем, решения задачи Коши для гиперболических уравнений допускают аналогичное представление. Подобные приложения привели к развитию теорий сингулярных интегральных операторов, псевдодифференциальных операторов и интегральных операторов Фурье. В отличие от задачи Коши для эллиптических уравнений, особенности решений гиперболических уравнений распространяются вдоль особенностей ядра Шварца оператора решения. В этом случае теории псевдодифференциальных операторов становится недостаточно, и на ее место приходит общая теория интегральных операторов Фурье. В настоящей работе мы обсудим теорию непрерьвности невырожденных интегральных операторов Фурье в $L^{p}$ и в других функциональных пространствах. Под непрерывностью в $L^{p}$ (или из $L^{p}$ в $L^{p}$ ) мы будем понимать непрерьвность (линейного оператора) из $L_{\mathrm{comp}}^{p}$ в $L_{\mathrm{loc}}^{p}$. Так как оценки могут зависеть от структуры волнового фронта оператора, мы также обсудим некоторые связанные задачи теории особенностей волновых фронтов. Особенности, возникающие в $L^{p}$-теории, являются частным случаем особенностей аффинных слоений, для которых мы выведем оценки на размерность множества существенных особенностей и проведем анализ его структуры.

Под интегральными операторами Фурье мы будем понимать интегральные операторы $T$, локально имеющие вид

$$
T u(x)=\int_{\mathbb{R}^{m}} \int_{\mathbb{R}^{N}} e^{i \Phi(x, y, \theta)} a(x, y, \theta) u(y) d \theta d y
$$


Здесь $a \in S^{\nu}\left(\mathbb{R}^{n} \times \mathbb{R}^{m} \times \mathbb{R}^{N}\right)$ - символ порядка $\nu$, т.е. гладкая функция, удовлетворяющая неравенству

$$
\left|\partial_{x, y}^{\beta} \partial_{\xi}^{\alpha} a(x, y, \theta)\right| \leqslant C(\alpha, \beta, K)(1+|\theta|)^{\nu-|\alpha|}
$$

на любом компактном множестве $K$ и для любых мультииндексов $\alpha$ и $\beta$. Фазовая функция $\Phi$ - гладкая, положительно однородная по $\theta$ с порядком один и невырожденная. Отметим сразу, что в разделе 2.1 мы приведем более обшее определение для произвольных гладких многообразий и символов класса $S_{\rho}^{\mu}$. Ядра Шварца операторов вида (1.1) называются распределениями Фурье. Волновой фронт распределения Фурье оператора $T$ задает геометрический инвариант оператора $T$. Действительно, множество

$$
W F(T)=\left\{\left(x, d_{x} \Phi(x, y, \theta), y, d_{y} \Phi(x, y, \theta)\right): d_{\theta} \Phi(x, y, \theta)=0\right\}
$$

в кокасательном расслоении $T^{*}\left(\mathbb{R}^{n} \times \mathbb{R}^{m}\right)$ не зависит от выбора фазовой функции $\Phi$. Если оснастить $T^{*}\left(\mathbb{R}^{n} \times \mathbb{R}^{m}\right)$ симплектической формой, то коническое множество в (1.3) становится лагранжевым подмногообразием кокасательного расслоения $T^{*}\left(\mathbb{R}^{n} \times \mathbb{R}^{m}\right)$. Одним из основных результатов глобальной теории интегральных операторов Фурье является обратное утверждение, которое удобнее сформулировать на языке многообразий. Именно, пусть $X$ и $Y$ - вешественные гладкие многообразия размерностей $n$ и $m$, и будем в дальнейшем предполагать, что $n=m$. Через $\sigma_{X}$ и $\sigma_{Y}$ обозначим стандартные симплектические формы на $T^{*} X$ и $T^{*} Y$ соответственно. Пусть $C$ - коническое лагранжево подмногообразие в $T^{*} X \backslash 0 \times T^{*} Y \backslash 0$, оснашенное симплектической формой $\sigma_{X} \oplus-\sigma_{Y}$. Тогда $C$ задает семейство интегральных операторов Фурье $T$ с $W F(T)=C$, локально имеющих вид (1.1). Множество $C$ назьвается каноническим отношением. Если зафиксировать порядок $\nu$ символа $a$ в (1.1), то множество операторов $T$ обозначается через $I^{\mu}(X, Y ; C)$, где $\mu=\nu+(N-n) / 2$. Приведем несколько важных примеров интегральных операторов Фурье. Если взять $n=m=N, a=1$ и

$$
\Phi(x, y, \theta)=\langle x-y, \theta\rangle
$$

то в правой части формулы (1.1) стоит композиция прямого и обратного преобразования Фурье в $\mathbb{R}^{n}$, так что в этом случае $T$ - тождественный оператор. Если фазовая функция $\Phi$ оператора $T$ имеет вид (1.4) и символ $a$ полиномиален по $\theta$, то $T$ задает дифференциальньй оператор с символом $a$. Если фазовая функция $\Phi$ оператора $T$ имеет вид (1.4) и символ $a$ произвольный, как в (1.2), то $T$ задает псевдодифференциальный оператор с символом $a$. Пространство псевдодифференциальных операторов порядка $\mu$ будем обозначать через $\Psi^{\mu}$. При решении волнового уравнения возникает фазовая функция $\Phi(x, y, \theta)=\langle x-y, \theta\rangle+|\theta|$. В разделе 5.2 мы приведем примеры операторов свертки и в разделе 6 операторы решений задачи Коши для гиперболических уравнений как интегральных операторов Фурье.

В настоящей статье нас будут интересовать свойства непрерьвности описанных операторов в различных функциональных пространствах. Наилучшее поведение демонстрируют псевдодифференциальные операторы. Если $P \in \Psi^{0}$ - псевдодифференциальный оператор нулевого порядка, то как линейный оператор $P$ непрерьвен из 
$L^{p}$ в $L^{p}$ для любых $p$ с $1<p<\infty$. Более того, псевдодифференциальный оператор $P \in \Psi^{\mu}$ порядка $\mu \in \mathbb{R}$ может быть расширен до непрерывного оператора из пространства Соболева $L_{k}^{p}$ в $L_{k-\mu}^{p}$ для любых $k \in \mathbb{R}, k \geqslant \mu$, и $1<p<\infty$. Также ничего не теряется в пространствах Липшица, операторы $P \in \Psi^{\mu}$ непрерывны из $\operatorname{Lip}(\gamma)$ в $\operatorname{Lip}(\gamma-\mu)$ для всех $\gamma>\mu$. Однако, для псевдодифференциальных операторов фазовая функция $\Phi$ имеет вид (1.4), и каноническое отношение $C$ равно конормальному расслоению к диагонали в $\mathbb{R}^{n} \times \mathbb{R}^{n}$. Для интегральных операторов Фурье структура $C$ гораздо сложнее, и их свойства непрерывности зависят от геометрических свойств канонического отношения.

Пусть $T \in I^{0}(X, Y ; C)$ - интегральный оператор Фурье нулевого порядка, ассоциированный с каноническим отношением $C$. Пусть $\pi_{X \times Y}, \pi_{X}, \pi_{Y}$ - стандартные проекции:

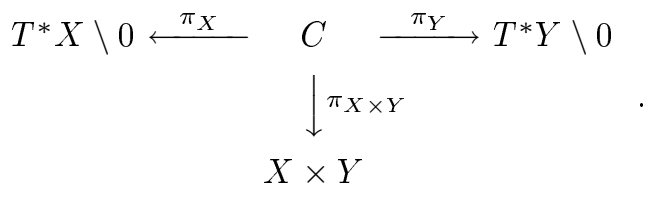

Оказывается, что для интегральных операторов Фурье свойства непрерьвности оператора $T$ зависят от особенностей проекций $\pi_{X \times Y}, \pi_{X}, \pi_{Y}$. Проекции $\pi_{X}, \pi_{Y}$ могут быть диффеоморфны только одновременно, и в этом случае для любого $\lambda_{0}=$ $\left(x_{0}, \xi_{0}, y_{0}, \eta_{0}\right) \in C$ существует симплектоморфизм $\chi$ (диффеоморфизм, сохраняюший симплектическую структуру) в окрестности точки $\left(y_{0}, \eta_{0}\right) \in T^{*} Y \backslash 0$ такой, что локально в окрестности $\lambda_{0}$ каноническое отношение $C$ принимает вид

$$
\{(x, \xi, y, \eta):(x, \xi)=\chi(y, \eta)\} .
$$

В этом случае $C$ локально задается графиком канонического симплектического отображения и называется локальны.м каноническим графиком или просто локальнымм графиком. Очевидно, условие, что $C$ - локальньй график, влечет за собой диффеоморфность проекций $\pi_{X}, \pi_{Y}$ с $C$ на $T^{*} X \backslash 0$ и $T^{*} Y \backslash 0$ соответственно. В частности, их размерности совпадают, $n=m$. Обратное тоже верно. В самом деле, предположим, что проекция $\pi_{Y}: C \rightarrow T^{*} Y \backslash 0$ - локальньй диффеоморфизм. Тогда локально каноническое отношение принимает вид (1.6) в системе координат $(y, \eta)$, и из условия, что $C$ - лагранжево многообразие для симплектической формы $\sigma_{X} \oplus-\sigma_{Y}$, вытекает, что форма $\sigma_{X} \oplus-\sigma_{Y}$ вырождается на $C$ и $\sigma_{Y}=\chi^{*}\left(\sigma_{X}\right)$. Последнее означает, что $\chi^{*}$ - симплектоморфизм и $C$ - локальный график.

В настояшей работе мы будем интересоваться случаем невырожденных интегральных операторов Фурье, т.е. операторов, у которых каноническое отношение - локальный график. Такие операторы возникают как операторы решения гиперболической задачи Коши. В этом случае отображение $\left.\pi_{X}\right|_{C} \circ \pi_{Y}^{-1}$ равно $\chi$ из (1.6) и задает локальный диффеоморфизм из $T^{*} Y \backslash 0$ в $T^{*} X \backslash 0$, и поэтому размерности $X$ и $Y$ совпадают. Операторы $T \in I^{0}(X, Y ; C)$ с локальньм граффиком $C$ непрерывны в $L^{2}$ ([14], $[23],[24])$. Доказательство основано на том, что каноническое отношение оператора $T \circ T^{*}-$ конормальное расслоение к диагонали в $X \times X$. Следовательно, $T \circ T^{*}$ - псевдодифференциальный оператор нулевого порядка и поэтому непрерывен в $L^{2}$. 
В общем, при $1<p<\infty, p \neq 2$, интегральные операторы Фурье нулевого порядка не непрерьвны в $L^{p}$.

С точки зрения непрерьвности интегральных операторов Фурье в $L^{p}$ псевдодифференциальные операторы и операторы, возникаюшие как решения волнового уравнения, - двапротивоположных случая. Фазовая функция последних имеет вид $\langle x-y, \xi\rangle+$ $|\xi|$ в $\mathbb{R}^{n}$, и Литтман ([31]) показал, что соответствующие операторы $T \in I^{\mu}(X, Y ; C)$ не непрерьвны в $L^{p}$ при $\mu>-(n-1)|1 / p-1 / 2|$. Непрерьвность в $L^{p}$ решений гиперболических задач Коши для уравнений волнового типа исследовалась во множестве работ ([58], [40], [36], [3]). Оценки в пространствах Липшица и $L^{p}$ для решений волнового уравнения на компактных многообразиях были получены в [7], и частные результаты для гиперболических уравнений с дополнительными условиями выпуклости были получены в [62]. Обшие результаты о непрерывности в $L^{p}$ были доказаны в [53]. Опишем их. Операторы $T \in I^{\mu}(X, Y ; C)$ непрерывны из $L_{\text {comp }}^{p} L_{\text {loc }}^{p}$, если $\mu \leqslant-(n-1)|1 / p-1 / 2|$. Этот порядок точен для эллиптических операторов $T$, для которых $\left.d \pi_{X \times Y}\right|_{C}$ имеет полный ранг, равньй $2 n-1$, хотя бы в одной точке. Это условие выполнено для решений задачи Коши для некоторых классов гиперболических операторов. Такая же потеря порядка на $(n-1)|1 / p-1 / 2|$ наблюдается в пространствах Соболева и Липшица (в пространствах Липшица $p=\infty$ ). Если обозначить $\alpha_{p}=(n-1)|1 / p-1 / 2|$, то операторы $T \in I^{\mu}(X, Y ; C)$ непрерывны из $L_{\alpha}^{p}$ в $L_{\alpha-\alpha_{p}-\mu}^{p}$ и из $\operatorname{Lip}(\alpha)$ в $\operatorname{Lip}\left(\alpha-\alpha_{\infty}-\mu\right)$. Доказательство непрерьвности в $L^{p}$ основано на методе комплексной интерполяции. Учитывая непрерьвность операторов нулевого порядка в $L^{2}$, достаточно показать, что операторы с порядком - $(n-1) / 2$ непрерывны из пространства Харди $H^{1}$ в $L^{1}$. Пространства Харди мы обсудим подробнее в разделе 2.2.

В общем, проекция $\pi_{X \times Y}$ удовлетворяет неравенству

$$
n \leqslant\left.\operatorname{rank} d \pi_{X \times Y}\right|_{C} \leqslant 2 n-1,
$$

так как $C$ - конус. Граничными примерами служат псевдодифференциальные операторы без потери порядка и решения волнового уравнения с потерей $(n-1)|1 / p-1 / 2|$ производных для непрерывности в $L^{p}$. Важной составляющей для определения наилучшего порядка для непрерьвности в $L^{p}$ является ранг в (1.7). Здесь существенно, чтобы $p \neq 2$, так как результаты в $L^{2}$ не зависят от ранга в (1.7). В [53] было получено следующее условие, гарантируюшее улучшение свойств непрерывности в $L^{p}$ операторов из $I^{\mu}(X, Y ; C)$. Будем говорить, что каноническое отношение $C$ удовлетворяет условию гладкой факторизации, если существует такое $k, 0 \leqslant k \leqslant n-1$, что проекцию $\pi_{X \times Y}$ можно локально гладко факторизовать на $C$ с помощью однородных отображений постоянного ранга $n+k$, сохраняющих уровни проекции $\pi_{X \times Y}$ размерности $n-k$. Более точно, для любого $\lambda_{0}=\left(x_{0}, \xi_{0}, y_{0}, \eta_{0}\right) \in C$ сушествуют коническая окрестность $U_{\lambda_{0}}$ точки $\lambda_{0}$ в $C$ и гладкое однородное с порядком 0 отображение $\pi_{\lambda_{0}}: C \cap U_{\lambda_{0}} \rightarrow C$ такие, что вьполнено

$$
\begin{gathered}
\operatorname{rank} d \pi_{\lambda_{0}} \equiv n+k, \\
\left.\pi_{X \times Y}\right|_{C \cap U_{\lambda_{0}}}=\pi_{X \times Y} \circ \pi_{\lambda_{0}} .
\end{gathered}
$$

При вьполнении условия гладкой факторизации операторы из $I^{\mu}(X, Y ; C)$ непрерывны из $L_{\text {comp }}^{p}$ в $L_{\text {loc }}^{p}$ при $1<p<\infty$ и $\mu \leqslant-k|1 / p-1 / 2|$. Мы обсудим точность этих порядков в разделе 7 и приведем обшие результаты для класса $I_{\rho}^{\mu}$ в разделе 2.3. Условие 
гладкой факторизации не является необходимым для непрерывности в $L^{p}$ операторов $T \in I^{\mu}$ с $\mu \leqslant-k|1 / p-1 / 2|$. Некоторые примеры показывают, что непрерывность возможна при нарушении условия факторизации ([52]).

Ослабление условия факторизации является открытой проблемой. Наилучшим результатом явилось бы доказательство того, что операторы $T \in I^{\mu}(X, Y$; $C$ ) не-

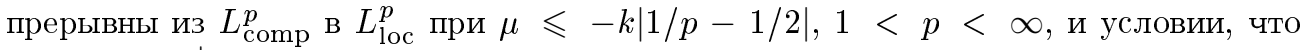
$\left.\operatorname{rank} d \pi_{X \times Y}\right|_{C} \leqslant n+k$. Отметим также, что доказательство результата при $k=n-1$ основано на непрерьвности из $H^{1}$ в $L^{1}$ операторов порядка $-(n-1) / 2$. Можно предположить, что такие операторы также слабо непрерывны в $L^{1}$, однако доказательство этого факта неизвестно. Однако, условие факторизации любопытно само по себе и допускает интересные обобшения, которые мы исследуем в разделах 3 и 4 .

Условие гладкой факторизации тривиально вьполнено в случае псевдодифференциальных операторов с $k=0$ и в случае максимального ранга с $k=n-1$, при котором в качестве $\pi_{\lambda_{0}}$ можно взять проекцию в коническом направлении. Оказьвается, что при некоторых естественных условиях на каноническое отношение $C$, выполненных в наиболее важных случаях, очень сложно продемонстрировать нарушение условия гладкой факторизации.

Опишем геометрическое значение условия гладкой факторизации. На гладком подмногообразии $\Sigma^{\infty}$ множества $\Sigma=\pi_{X \times Y}(C)$ каноническое отношение имеет вид конормального расслоения $N^{*} \Sigma^{\infty}$ к $\Sigma^{\infty}$. Множество $\Sigma$ является сингулярным носителем интегрального ядра Шварца оператора $T$. Конормальное расслоение $N^{*} \Sigma^{\infty} \mathrm{K}$ $\Sigma^{\infty} \subset X \times Y$ определяется с помошью

$$
\begin{aligned}
& N^{*} \Sigma^{\infty}=\left\{(x, \xi, y, \eta) \in T^{*}(X \times Y):(x, y) \in \Sigma^{\infty}\right. \\
&\left.\xi(\delta x)+\eta(\delta y)=0, \quad \forall(\delta x, \delta y) \in T_{(x, y)} \Sigma^{\infty}\right\} .
\end{aligned}
$$

Таким образом, диаграмма

$$
\begin{gathered}
N^{*} \Sigma^{\infty} \subset C \\
\downarrow_{\Sigma^{\infty}} \pi_{X \times Y}
\end{gathered}
$$

задает гладкое локальное слоение над $\Sigma^{\infty}$ с аффинными слоями. В этих терминах условие факторизации эквивалентно условию, что гладкое слоение $N^{*} \Sigma^{\infty}$ над $\Sigma^{\infty}$ допускает гладкое расширение как слоение $C$ над $\Sigma$, задаваемое с помощью уровней отображения $\pi_{\lambda_{0}}$. В разделах 3 и 4 мы проанализируем это условие с разными степенями гладкости слоения. Однако, анализ становится гораздо богаче, если предположить аналитичность описанного слоения над $\Sigma^{\infty}$. Условие аналитичности естественно и почти всегда выполнено. В самом деле, если критическая точка фазовой функции вырождается с конечным порядком, то можно выбрать карту в окрестности критической точки, в которой фазовая функция полиномиальна. С другой стороны, сушествует очень мало бесконечно вырожденных критических точек, так как для них коэффициенты Тейлора разложения в ряд фазовой функции удовлетворяют бесконечному числу независимых алгебраических уравнений. Сушествует также иная причина предположить аналитичность. Дело в том, что изучение свойств интегральных операторов Фурье часто сводится к асимптотике (при $\lambda \rightarrow \infty$ ) осциллирующих интегралов вида 
$\int e^{i \lambda \phi} \psi$. Асимптотика существенно зависит только от первых коэффициентов ряда Тейлора: наивысшая степень в асимптотическом приближении соответствует первому ненулевому члену ряда Тейлора фазовой функции ([8], [2]). В частности, условие аналитичности удовлетворено в приложениях к гиперболическим дифференциальным операторам с аналитическими коэффициентами. Свойства осциллируюших интегралов с аналитическими фазовыми функциями изучались в [2], и соответствуюшие геометрические конструкции можно найти в [24], [12]. Теорию особенностей волновых фронтов можно найти в [1]. При аналитичности будем называть условие гладкой факторизации условием голоморфной факторизации, если все отображения в (1.8) аналитичны (и поэтому голоморфны после продолжения в комплексную область). Мы покажем, что при аналитичности условие голоморфной факторизации вьполнено в ряде важных случаев. Некоторые результаты можно найти в [47] и [48]. Сушествуют альтернативные представления интегральных операторов Фурье, используюшие комплекснозначные фазовые функции. Они были изучены в [33], глобальные параметризации были получены в [30]. Обзор основной теории можно найти в [12].

Результаты о точности порядков для уравнений типа волнового уравнения можно найти в [36], [40], [53] в случае, когда $\left.\operatorname{rank} d \pi_{X \times Y}\right|_{C}=2 n-1$ хотя бы в одной точке. Для сушественно однородных символов $\left(S_{\rho}^{\mu}\right.$ с $\left.\rho=1\right)$ мы обобшим результаты на точность на произвольньй ранг. Мы покажем, что порядок $-k|1 / p-1 / 2|$ точен для произвольных эллиптических интегральных операторов $Ф$ урье с rank $\left.d \pi_{X \times Y}\right|_{C} \leqslant n+k$. В качестве следствия мы покажем, что эллиптические операторы небольших по абсолютной величине отрицательных порядков, непрерьвные в $L^{p}$ или из $L^{p}$ в $L^{q}$, можно получить из псевдодифференциальных операторов композицией с интегральными операторами Фурье, индуцированными гладкой заменой координат. Некоторые результаты появились в [49], и в настояшей работе мы опишем их в разделе 7 . Там же мы вкратце упомянем случай произвольного $\rho$, в котором точность порядков для произвольных эллиптических операторов при ограничении на ранг проекции $\left.\pi_{X \times Y}\right|_{C}$ в целом не доказана. В разделе 5 мы приведем примеры нарушения условия факторизации в обшем случае и в случае канонических отношений, соответствуюших операторам, коммутирующим со сдвигами.

Опишем вкратце условие гладкой факторизации в терминах фазовой функции интегрального оператора Фурье. В дальнейшем мы будем заменять частотную переменную $\theta$ на $\xi$ в случаях, когда размерности $X, Y$ и частотного пространства $\Xi$ совпадают. По теореме о выборе производяшей функции (теорема 2.3 ниже) можно полагать, что фазовая функция $\Phi$ оператора $T \in I^{\mu}(X, Y ; C)$ имеет вид

$$
\Phi(x, y, \xi)=\langle x, \xi\rangle-\phi(y, \xi) .
$$

Волновой фронт равен

$$
\Lambda_{\Phi}=\left\{\left(\nabla_{\xi} \phi(y, \xi), \xi, y, \nabla_{y} \phi(y, \xi)\right)\right\}
$$

и $C=\Lambda_{\Phi}^{\prime}=\left\{(x, \xi, y,-\eta):(x, \xi, y, \eta) \in \Lambda_{\Phi}\right\}$. Условие локального графика эквивалентно условию

$$
\operatorname{det} \phi_{y \xi}^{\prime \prime}(y, \xi) \neq 0
$$


на носителе символа оператора $T$. Отображение

$$
\gamma(y, \xi)=Y \times \Xi \ni(y, \xi) \mapsto\left(\nabla_{\xi} \phi(y, \xi), \xi, y, \nabla_{y} \phi(y, \xi)\right) \in T^{*} X \times T^{*} Y
$$

задает диффеоморфизм из $Y \times \Xi_{\text {в }} C$. Уровни отображения $\pi_{X \times Y}: C \rightarrow X \times Y$ соответствуют ядрам линейного отображения $\left.d \pi_{X \times Y}\right|_{C}$ или ядрам отображения $d \pi_{X \times Y} \circ d \gamma$. Легко показать, что

$$
\operatorname{ker} d \pi_{X \times Y} \circ d \gamma(y, \xi)=\left(0, \operatorname{ker} \frac{\partial^{2} \phi}{\partial \xi^{2}}(y, \xi)\right)
$$

Таким образом, слоение (1.11) сводится к слоению, задаваемому ядрами ker $\phi_{\xi \xi}^{\prime \prime}(y, \xi)$ или уровнями отображения $(y, \xi) \mapsto \nabla_{\xi} \phi(y, \xi)$ на множестве с максимальным рангом $\phi_{\xi \xi}^{\prime \prime}$. Простой пример нарушения условия факторизации возможен уже в $\mathbb{R}^{3}$. Для функции

$$
\phi(y, \xi)=\langle y, \xi\rangle+\frac{1}{\xi_{3}}\left(y_{1} \xi_{1}+y_{2} \xi_{2}\right)^{2}
$$

слои в кокасательном расслоении (т.е. уровни $\nabla \phi(y, \xi)$ по $\xi)$ - прямые линии в направлении $y_{2} / y_{1}$. Видно, что соответствуюшее слоение не непрерьвно в нуле. В случае, когда фазовая функция вешественно аналитична, мы сконцентрируемся на ее комплексном продолжении и на голоморфном отображении

$$
(y, \xi) \mapsto \nabla_{\xi} \phi(y, \xi) .
$$

Опишем подробно возникающую задачу в терминах аффинных слоений.

1.2. Слоения с аффинными слоями. Сформулируем сначала инвариантную версию задачи, соответствующую анализу коммутирующих со сдвигами интегральных операторов Фурье. В этом случае $\phi(y, \xi)=\langle y, \xi\rangle-H(\xi)$. Функции $H: V \rightarrow \mathbb{R}$ на открытом множестве $V$ в $\mathbb{R}^{n}$ обладают следующим свойством. Максимальный ранг $k$ гессиана $D^{2} H(\xi)$ строго меньше чем $n$, и точки $\xi$ с максимальных рангом $\operatorname{rank} D^{2} H(\xi)=k$ образуют открытое множество $U$. Одно из интересных свойств градиента $\Gamma: \xi \mapsto \nabla H(\xi)$ заключается в том, что для любой точки $\xi \in U$ поверхность уровня $\Gamma^{-1}(\Gamma(\xi))$ локально в окрестности $\xi$ совпадает с аффиинным пространством $\xi+\operatorname{ker} D^{2} H(\xi)$. Если $H$-вешественно аналитическая функция, то таким же свойством обладает и голоморфное расширение Г градиента $H$ в некоторую открытую окрестность множества $V$ в $\mathbb{C}^{n}$. Упомянутое свойство мотивирует изучение голоморфных отображений Г со свойствами (A1)-(А3), сформулированными ниже.

Пусть $\Gamma$ - голоморфное отображение из открытого связного множества $\Omega \subset \mathbb{C}^{n}$ в $\mathbb{C}^{p}$. Через $D \Gamma(\xi) \in \mathbb{C}^{p \times n}$ обозначим якобиан отображения $\Gamma$. Строки $D \Gamma$ имеют вид $D_{\xi} \Gamma_{i}(\xi)=\left(\nabla_{\xi} \Gamma_{i}\right)^{T}$ для $1 \leqslant i \leqslant p$. Выберем $k$ так, чтобы

(A1) $\operatorname{rank} D \Gamma(\xi) \leqslant k$ для всех $\xi \in \Omega$;

(A2) $\exists \xi \in \Omega \mathrm{c} \operatorname{rank} D \Gamma(\xi)=k$. 
Так как нас будут интересовать уровни отображения Г, можно предположить, что $k \leqslant n-1$. Множество $\Omega$ допускает стратификацию как объединение непересекаюшихся множеств $\Omega^{(i)}$ точек $\xi \in \Omega \mathrm{c} \operatorname{rank} D \Gamma(\xi)=i, i=0, \ldots, k$. Множество $\Omega \backslash \Omega^{(k)}$, где ранг $D \Gamma(\xi)<k,-$ аналитическое подмножество в $\Omega$, и на открытом плотном множестве $\Omega^{(k)}$ отображение

$$
\varkappa: \xi \mapsto \operatorname{ker} D \Gamma(\xi)
$$

голоморфно из $\Omega^{(k)}$ в грассманиан $\mathbb{G}_{n-k}\left(\mathbb{C}^{n}\right)$. Через $\Omega^{\operatorname{sing}}$ обозначим мно жество существенных особенностей отображсени $\varkappa$, т.е. множество точек $\xi \in \Omega \backslash \Omega^{(k)}$, для которых $\varkappa$ не допускает голоморфного продолжения через $\xi$. Отображение $\varkappa$ регулярно (голоморфно) в точках $\xi \in \Omega^{(k)}$, и такие точки называются регулярными. Множество $\Omega \backslash \Omega^{(k)}$ состоит из существенных особенностей в $\Omega^{\operatorname{sing}}$ и устранимых особенностей в $\Omega \backslash\left(\Omega^{(k)} \cup \Omega^{\text {sing }}\right)$.

Дополнительное предположение на Г будет состоять в том, что аффинные подпространства $\xi+\varkappa(\xi)$ задают слоение в $\Omega^{(k)}$. Объясним подробнее, что мы понимаем под этим. В точках $\xi \in \Omega^{(k)}$ по теореме о неявной функции поверхности уровня $\Gamma^{-1}(\Gamma(\xi))$ представляют собой гладкие аналитические поверхности размерности $n-k$. Касательное пространство к $\Gamma^{-1}(\Gamma(\xi))$ в $\xi$ задается с помощью $\xi+\varkappa(\xi)$. Условие, что $\varkappa$ задает слоение, заключается в том, что поверхность уровня совпадает со своим касательным пространством в $\xi$ для всех $\xi \in \Omega^{(k)}$.

(А3) Для каждого $\xi \in \Omega^{(k)}$ сушествует окрестность $U$ точки $\xi$ такая, что аффинное пространство $\xi+\varkappa(\xi)$ совпадает с поверхностью уровня $\Gamma^{-1}(\Gamma(\xi))$ в $U$.

Таким образом, Г постоянна на $\xi+\varkappa(\xi)$, что также эквивалентно условию, что $\varkappa(\xi+\zeta)=\varkappa(\xi)$ для всех $\xi \in \Omega^{(k)}$ и $\zeta \in \varkappa(\xi)$ с $\xi+\zeta \in \Omega^{(k)}$ (см. утверждение 3.18 ниже). Так как мы заинтересованы в локальных свойствах отображения $\varkappa$, будем всегда предполагать выпуклость $\Omega$. Тогда множество $(\xi+\varkappa(\xi)) \cap \Omega$ связно, $Г$ постоянна на всем $(\xi+\varkappa(\xi)) \cap \Omega$ и $\varkappa(\xi)=\varkappa(\eta)$ для всех $\eta \in(\xi+\varkappa(\xi)) \cap \Omega^{(k)}$. Так, условие (А3) влечет за собой глобальную форму:

$\left(\mathrm{A} 3^{\prime}\right)$ Для любой точки $\xi \in \Omega^{(k)}$ аффиннное пространство $(\xi+\varkappa(\xi))$ совпадает с поверхностью уровня $\Gamma^{-1}(\Gamma(\xi))$ на всем множестве $\Omega$.

Отображение $\varkappa$ голоморфно продолжимо на открытое множество $\Omega \backslash \Omega^{\text {sing }}$, содержашее $\Omega^{(k)}$. Это продолжение будем также обозначать через $\varkappa$. Таким образом, для всех $\xi \in \Omega \backslash \Omega^{\operatorname{sing}}$ отображение Г постоянно на $(\xi+\varkappa(\xi)) \cap \Omega$. Если для двух точек $\xi, \eta \in \Omega \backslash \Omega^{\text {sing }}$ пересечение $(\xi+\varkappa(\xi)) \cap(\eta+\varkappa(\eta)) \cap\left(\Omega \backslash \Omega^{\text {sing }}\right)$ непусто, то $\varkappa(\xi)=\varkappa(\eta)$. Это позволяет ввести отношение эквивалентности на $\Omega \backslash \Omega^{\operatorname{sing}}$. Будем говорить, что точки $\xi$ и $\eta$ эквивалентны, $\xi \sim \eta$, если $\eta \in \xi+\varkappa(\xi)$. Элемент $\varkappa(\xi)=\varkappa(\eta)$ задает их обший класс эквивалентности. Фактор-пространство $\left(\Omega \backslash \Omega^{\text {sing }}\right) / \sim$ - гладкое аналитическое многообразие размерности $k$, и проекция $\xi \mapsto(\xi+\varkappa(\xi)) \cap\left(\Omega \backslash \Omega^{\operatorname{sing}}\right)$ задает аналитическое погружение. В этом смысле $(\xi+\varkappa(\xi)) \cap\left(\Omega \backslash \Omega^{\operatorname{sing}}\right)$ с $\xi \in \Omega \backslash \Omega^{\operatorname{sing}}$ задают гладкое слоение в $\Omega \backslash \Omega^{\text {sing }}$. Отображение $\Gamma$ факторизуется через это слоение (см. начало раздела 3.3 ). В связи с этим для краткости будем назьвать само отображение $\varkappa$ слоением, имея в виду задаваемое им слоение.

Простейший пример сингулярного слоения можно привести в $\Omega=\mathbb{C}^{n}$, взяв в качестве слоев открытые лучи из нуля в радиальных направлениях. Очевидно, такое 
слоение регулярно (аналитично) во всех точках кроме нуля, и множество его существенных особенностей $\Omega^{\text {sing }}=\{0\}$. Однако, как мы покажем в разделе 3 , в этом случае не существует голоморфного отображения $\Gamma$, для которого лучи задаются ядрами якобиана $D \Gamma$ (даже локально).

Заметим, что условия (A1)-(А3) можно сформулировать в большей общности. В дальнейшем мы покажем мероморфность отображения $\varkappa$, что позволяет сформулировать аналогичную задачу в категории мероморфных отображений. Для достижения еще большей общности можно работать с морфизмами $\varkappa$ из аналитического пространства $X$ в (компактное) аналитическое пространство $Y$ такими, что $\varkappa$ голоморфно на дополнении в $X$ к некоторому аналитическому множеству строго меньшей размерности. Однако, в настоящей работе нас интересуют свойства отображения $\varkappa$ при заданном фиксированном отображении Г. Поэтому мы будем избегать общего языка категорий и сосредоточимся на локальных свойствах $\varkappa$.

1.3. Формулировка проблемы для параметризованных слоений. Рассмотрим кратко более обшую постановку с зависимостью от $y$. Пусть $Г$ - голоморфное отображение из связного открытого множества $\Omega \subset \mathbb{C}^{m} \times \mathbb{C}^{n}$ в $\mathbb{C}^{p}$. Пусть $k<n$ - максимальньй ранг якобиана $D_{\xi} \Gamma(y, \xi)$ на $\Omega$,

$(\mathrm{P} 1)-(\mathrm{P} 2) \max _{(y, \xi) \in \Omega} \operatorname{rank} D_{\xi} \Gamma(y, \xi)=k$.

Множество $\Omega^{(k)}$ точек $(y, \xi)$, где ранг $k$ достигается, открыто и плотно в $\Omega$. Отображение

$$
\varkappa:(y, \xi) \mapsto \operatorname{ker} D_{\xi} \Gamma(y, \xi)
$$

голоморфно из $\Omega^{(k)}$ в грассманиан $\mathbb{G}_{n-k}\left(\mathbb{C}^{n}\right)$, состоящий из $(n-k)$-мерных линейных подпространств в $\mathbb{C}^{n}$. Через $\Omega^{\text {sing }}$ обозначим множество существенных особенностей $\varkappa$, т.е. множество точек $(y, \xi) \in \Omega$ таких, что $\varkappa$ не допускает голоморфного расширения в окрестность точки $(y, \xi)$ в $\Omega$. Очевидно, множества $\Omega^{(k)}$ и $\Omega^{\operatorname{sing}}$ не пересекаются. Следующее условие гарантирует линейность по $\xi$ поверхностей уровня отображения $Г$ :

(Р3) Для всех $(y, \xi) \in \Omega^{(k)}$ афффинное пространство $(y, \xi)+(0, \varkappa(y, \xi))$ локально совпадает с поверхностью уровня $\Gamma^{-1}(\Gamma(y, \xi))$ через $(y, \xi)$.

Как и условия (A1)-(А3), (Р3) означает, что $\varkappa$ задает локальное голоморфное слоение в $\Omega \backslash \Omega^{\text {sing }}$. Примеры типа (1.12) с $\Gamma=\nabla \phi$ показьвают сушествование слоений с существенными особенностями. Если мы зафиксируем некоторое $y$, либо будем брать $Г$, не зависящие от $y$, то условия (P1)-(Р3) эквивалентны условиям (А1)-(А3) вьше. Оказьвается, что простые примеры, как в (1.12), невозможны в задаче (A1)-(A3), и анализ становится гораздо интереснее. Например, одним из необходимых условий для того, чтобы аналитическое множество $\Omega^{\text {sing }}$ было множеством сушественных особенностей отображения $\varkappa$, соответствующего голоморфному отображению Г, является следующая оценка на размерность:

$$
\max \{k-1, n-k+1\} \leqslant \operatorname{dim}_{\xi} \Omega^{\operatorname{sing}} \leqslant n-2 .
$$

В частности, $\Omega^{\text {sing }}$ не может содержать изолированных точек.

Со слоениями, возникающими таким образом, связан ряд интересных вопросов. Так, при заданном слоении $\varkappa_{0}$ на открытом плотном множестве $\Omega_{0}$ в $\Omega$ определить, 
сушествует ли голоморфное отображение $\Gamma$, удовлетворяющее условиям (A1)-(A3), для которого слоение, задаваемое отображением $\varkappa$, совпадает со слоением, заданным отображением $\varkappa_{0}$ на $\Omega_{0} \cap \Omega^{(k)}$. Вопрос построения фазовой функции $\phi$ при заданном $\Gamma=\nabla_{\xi} \phi$ вызывает дополнительные трудности. В разделе 4 мы получим необходимые и достаточные условия в терминах системы дифференциальных уравнений с коэффициентами, соответствующими данному слоению. Однако, даже если слоение регулярно (аналитично), нет гарантии, что решение построенной системы уравнений достаточно регулярно. Кроме того, система зависит от выбора локальной системы координат в грассманиане. Было бы интересно получить инвариантную характеризацию результатов раздела 4 , а также их обобщения на пространства большей размерности. Результаты раздела 3 (например, теоремы 3.9-3.12) описьвают возможные размерности множества $\Omega^{\operatorname{sing}}$ для задачи (A1)-(A3), а также дают некоторое представление о его структуре. Было бы интересно исследовать его дальнейшие свойства, особенно в случае слоений градиентного типа. Например, в разделе 4.4 мы приведем примеры слоений градиентного типа с непустым множеством $\Omega^{\operatorname{sing}}$ существенных особенностей. Однако во всех построенных нами примерах множество $\Omega^{\text {sing }}$ аффинно и его размерность равна $n-2$. Непонятно, является ли условие $\operatorname{dim} \Omega^{\operatorname{sing}}=n-2$ необходимым для слоений градиентного типа.

В виду обшности двух задач будем использовать одинаковые обозначения для встречающихся объектов. Во избежание недоразумений договоримся по умолчанию рассматривать задачу (A1)-(А3).

1.4. Некоторые приложения. Одним из основных приложений интегральных операторов Фурье является теория гиперболических уравнений в частных производных. Пусть оператор

$$
P\left(t, x, \partial_{t}, \partial_{x}\right)=\partial_{t}^{m}+\sum_{j=1}^{m} P_{j}\left(t, x, \partial_{x}\right) \partial_{t}^{m-j}
$$

строго гиперболический порядка $m$ на некотором множестве точек $(t, x) \in \mathbb{R}^{1+n}$. Гиперболичность означает, что главный символ $p(t, x, \tau, \xi)$ оператора $P$ (которьй полиномиален по $\tau$ с порядком $m$ и также обозначается через $\sigma_{P}$ ) имеет $m$ различных вещественных корней $\tau_{j}$ по $\tau$. В этом случае задача Коши

$$
\left\{\begin{array}{l}
P u(t, x)=0, \quad t \neq 0, \\
\left.\partial_{t}^{j} u\right|_{t=0}=f_{j}(x), \quad 0 \leqslant j \leqslant m-1,
\end{array}\right.
$$

разрешима, и при небольших $t$ ее решение представимо в виде суммы эллиптических интегральных операторов Фурье $T_{t}^{j l}$ :

$$
u(t, \cdot)=\sum_{j=1}^{m} \sum_{l=0}^{m-1} T_{t}^{j l} f_{l},
$$

где каждый оператор $T_{t}^{j l}$ гладко зависит от $t$, имеет порядок $-l$ и зависит от корня $\tau_{j}(t, x, \xi)$. Подробно эта конструкция будет описана в разделе 6 . Отметим, что операторы $T_{t}^{j l}$ удовлетворяют условию локального графиика и из описанных ниже результатов (см., например, теорему 2.9 и [53]) следует, что для небольших $t$ и $1<p<\infty$ решение задачи Коши $u(t, \cdot) \in L_{\text {loc }}^{p}$, если данные Коши $f_{l}$ принадлежат пространствам 
Соболева $f_{l} \in\left(L_{\alpha_{p}-l}^{p}\right)$ comp, где $\alpha_{p}=(n-1)|1 / p-1 / 2|$. Таким образом, в $L^{p}$ происходит потеря гладкости для решений на $\alpha_{p}$ производных. Методом стационарной фазы можно показать, что порядок $\alpha_{p}$ точен в ряде случаев, например, для уравнений волнового типа с переменными коэффициентами (при $m=2$ ). Он также точен, если наложить дополнительное условие, что для почти всех $t$ хотя бы один из корней $\tau_{j}(t, x, \xi)$ эллиптический по $\xi$, т.е. $\tau_{j}(t, x, \xi) \neq 0$ при $\xi \neq 0$. Однако, в ряде случаев порядок $\alpha_{p}$ можно улучшить. Обозначим через $k$ минимальное натуральное число, для которого ранги стандартных проекций с канонического отношения оператора $T_{t}^{j l}$ в $\mathbb{R}^{n} \times \mathbb{R}^{n}$ не превосходят $n+k$ для всех $j$ и $l$. Подробно эти проекции описаны в разделе 1.1 , в нашем случае проекция $\pi_{X \times Y}$ в (1.7) удовлетворяет условию rank $\left.d \pi_{X \times Y}\right|_{C_{t}^{j l}} \leqslant n+k$, где $X$ и $Y$ суть (открытые множества в) $\mathbb{R}^{n}$ и $C_{t}^{j l}$ - канонические отношения операторов $T_{t}^{j l}$. В терминах фазовых функций это означает, что если $\Phi_{j}$ - решение уравнения эйконала $(\partial / \partial t) \Phi_{j}(t, x, \xi)=\tau_{j}\left(t, x, \nabla_{x} \Phi_{j}\right)$ с начальным условием $\Phi_{j}(0, x, \xi)=\langle x, \xi\rangle$, то rank $\partial_{\xi \xi}^{2} \Phi_{j}(t, x, \xi) \leqslant k$ для всех $j, x, \xi$. В этом случае можно показать, что порядок $\alpha_{p}=k|1 / p-1 / 2|$ точен, т.е. условие, что при некотором $\alpha u(t, \cdot) \in L_{\mathrm{loc}}^{p}$ для любых $f_{l} \in\left(L_{\alpha-l}^{p}\right)_{\text {comp }}$, влечет $\alpha \geqslant \alpha_{p}=k|1 / p-1 / 2|$. Обратное известно только в ряде случаев. Для операторов с постоянными коэффициентами (в частности) обратный результат будет доказан в разделе 6 для $k \leqslant 2$. Полная картина $L^{p}$ свойств для таких операторов возникает в 5-мерном пространстве (см. раздел 6 ). Для $3 \leqslant k \leqslant n-2$ точные $L^{p}$-оценки, в целом, не доказаны. В случае $k=0$, когда потери гладкости решений нет (т.е. свойства регулярности решений совпадают со свойствами регулярности решений эллиптической задачи Коши, $\alpha_{p}=0$ и, следовательно, $k=0$ ), главный символ оператора $P$ принимает специальную форму, его корни $\tau_{j}$ линейны по $\xi$ (см. теорему 6.4).

В качестве другого важного приложения отметим операторы свертки с мерами и преобразования Радона. Пусть $X$ и $Y$ - гладкие многообразия, и пусть $S$ - гладкое подмногообразие в $X \times Y$. Для $x \in X$ через $S_{x}$ обозначим множество точек $y \in Y$, для которых $(x, y) \in S$. Пусть $\sigma$ - гладкая мера на $S$, индуцирующая меру $\sigma_{x}$ на $S_{x}$. Преобразование Радона, соответствующее $S$ и $\sigma$, определенное с помощью

$$
R f(x)=\int_{S_{x}} f d \sigma_{x}
$$

задает интегральный оператор $R: C_{0}^{\infty}(Y) \rightarrow \mathscr{D}^{\prime}(X)$. Ядро Шварца оператора $R-$ мера Дирака, сосредоточенная и равная $\sigma$ на $S$. Так как мы заинтересованы в локальных свойствах операторов, предположим, что множество $S$ компактно. Пусть $\operatorname{dim} S=\operatorname{dim} X+\operatorname{dim} Y-d$. Локально $S$ можно задать с помощью $d$ уравнений

$$
h_{1}(x, y)=\cdots=h_{d}(x, y)=0
$$

где функции $h_{j}$ гладкие и их градиенты линейно независимы на $S$. Тогда оператор $R$ можно рассматривать как интегральный оператор Фурье с фазовой функцией

$$
\sum_{j=1}^{d} \theta_{j} h_{j}(x, y)
$$


Каноническое отношение оператора $S$ принимает вид конормального расслоения $N^{*} S$. Условие локального графиика означает, что $\operatorname{dim} X=\operatorname{dim} Y$ и проекции $\pi_{X}, \pi_{Y}$ из $N^{*} S$ в $T^{*} X$ и $T^{*} Y$ локально диффеоморфны. В этом случае применимы $L^{p}$-оценки последуюших разделов. Так как $S$ - гладкое многообразие, то условие гладкой факторизации для $N^{*} S$ выполнено, откуда следуют точные оценки. Результаты зависят от порядка оператора $R$ как интегрального оператора Фурье. Для простоты предположим, что $R f=f * \sigma$, где мера $\sigma$ сосредоточена на некотором (гладком) подмногообразии $\Sigma$ в $\mathbb{R}^{n}$. Связь $R$ с интегральньми операторами Фурье более подробно описана в разделе 5.2. Сейчас ограничимся несколькими замечаниями. Порядок $R$ как интегрального оператора Фурье связан с порядком убьвания преобразования Фурье $\widehat{\sigma}$ на бесконечности. Этот порядок зависит от кривизны $\Sigma$. Пусть $\Sigma-$ гиперповерхность $(\operatorname{dim} \Sigma=n-1)$ с ненулевой кривизной Гаусса во всех точках,$d \sigma=\psi d \mu$, где $d \mu$ индуцируется на $S$ мерой Лебега и $\psi \in C_{0}^{\infty}\left(\mathbb{R}^{n}\right)$. Тогда можно показать, что преобразование Фурье меры $\sigma$ удовлетворяет неравенству $|\widehat{\sigma}(\xi)| \leqslant C|\xi|^{-(n-1) / 2}$. Отсюда вытекает, что $R-$ интегральньй оператор Фурье порядка $-(n-1) / 2$. Сушествуют различные обобщения оценки на $(n-d)$-мерные многообразия $\Sigma$ конечного типа. Грубо говоря, $\Sigma$ имеет тип $m$, если порядок касания $\Sigma$ с аффинными гиперплоскостями не превосходит $m$. В этом случае $|\widehat{\sigma}(\xi)| \leqslant C|\xi|^{-1 / m}$. Если многообразие $\Sigma$ аналитично, то из приведенной оценки следует, что $\Sigma$ имеет тип $m$, так как иначе функция $\widehat{\sigma}$ постоянна в направлениях, ортогональных касательному пространству к $\Sigma$ в точке касания бесконечной кратности. Более подробное обсуждение, а также другие формы преобразования Радона можно найти, например, в [59], [18], [41], [42], [44]. Для нарушения условия гладкой факторизации необходимо (но не достаточно, см. замечание 2.23), чтобы $S$ не было гладким. В этом случае дополнительную сложность вызывает оценка роста функции $\widehat{\sigma}$ на бесконечности для определения порядка $R$ как интегрального оператора Фурье.

Условие локального графика выполнено в ряде важных случаев. Например, преобразования Радона вдоль гиперплоскостей в $\mathbb{R}^{n}$ удовлетворяют этому условию. Также операторы свертки с мерами, сосредоточенными на гиперповерхностях в $\mathbb{R}^{n}$ с ненулевой кривизной Гаусса, удовлетворяют условию локального графика. В качестве примера имеем операторы решений задачи Коши для волнового уравнения (см. вьше), где при фиксированном $t>0 S_{x}$ состоит из точек $y \in \mathbb{R}^{n} \mathrm{c}|x-y|=t$.

Отметим, наконец, что в некоторых приложениях каноническое отношение не является локальным графиком. Для преобразований Радона с $d>n / 2$ условие локального графика всегда нарушается (см., например, [41]). Для подмногообразий коразмерности больше чем 1 в обшем положении каноническое отношение $C$ складка Витни ([60], [43], [42]). Особенности проекций $\pi_{X}$ и $\pi_{Y}$ также возникают в приложениях к теории дифракций ([63], [64]), в теории преобразований Радона вдоль лучей ([17]) и в теории деформаций ([19], [17]). Сингулярные преобразования Радона и интегральные операторы Фурье с плотностями типа Кальдерона-Зигмунда приводят к вопросам непрерывности вырожденных интегральных операторов Фурье (для некоторых задач см. [20], [18], [42]). Во многих вырожденных случаях происходит потеря порядка для непрерьвности даже в $L^{2}$, и подобные результаты часто формулируются в терминах стратификации лагранжиана ([43], [44]). Определенная потеря непрерьвности происходит также в $L^{p}$ для преобразований Радона с каноническим отношением типа складки и другими особенностями. Однако такие вырожденные 
случаи выходят за рамки настоящей статьи.

Библиография в настоящей статье была подобрана с целью осветить некоторые аспекты теории регулярности интегральных операторов Фурье и связанных с этим геометрических задач и не претендует на полноту. Многие результаты статьи были получены автором во время работы над диссертацией в Утрехтском университете. Пользуясь случаем, хочу поблагодарить профессора Дюстермаата за многочисленные дискуссии и замечания.

\section{2. Оценки для интегральных операторов Фурье}

2.1. Интегральные операторы Фурье. В дальнейшем мы будем в основном интересоваться локальными свойствами интегральных операторов Фурье. Однако глобальные конструкции иногда облегчают анализ. Глобальная теория интегральных операторов Фурье была систематически развита в [23], [8], [24]. Хорошее изложение можно найти в [12], [13]. Много необходимых нам понятий описано в [32]. Глобальная теория основана на некоторых понятиях симплектической геометрии. Напомним основные определения.

Пусть $M$ - гладкое вешественное многообразие. Дифференшиальная 2-форма $\omega$ на $M$ называется симплектической, если $d \omega=0$ и для каждого $m \in M$ билинейная форма $\omega_{m}$ невырождена и антисимметрична на $T_{m} M$. Пары $\left(T_{m} M, \omega_{m}\right)$ и $(M, \omega)$ назьваются симплектическим линейным пространством и симплектическим многообразием соответственно. Пусть $X$ - гладкое вешественное $n$-мерное многообразие. Симплектическую форму $\sigma$ на кокасательном расслоении $T^{*} X$ многообразия $X$ можно задать следуюшим образом. Пусть $\pi: T^{*} X \ni(x, \xi) \mapsto x \in X$-стандартная проекция. Для $(x, \xi) \in T^{*} X$ отображения $D \pi_{(x, \xi)}: T_{(x, \xi)}\left(T^{*} X\right) \rightarrow T_{x} X$ и $\xi: T_{x} X \rightarrow \mathbb{R}$ линейны. Их композиция

$$
\alpha_{(x, \xi)}=\xi \circ D \pi_{(x, \xi)}
$$

- 1-форма на $T^{*} X$. Ее дифференциал $\sigma=d \alpha$ назьвается стандартной 2-формой на $T^{*} X$. Форма $\sigma$ - симплектическая и соответствует дифференциальной форме $\sum_{j=1}^{n} d p_{j} \wedge d q_{j}$ в механике. Можно показать, что любая симплектическая форма принимает такой вид в симплектических координатах. Все объекты существуют также на комплексных аналитических многообразиях.

Подмногообразие $\Lambda$ кокасательного расслоения $T^{*} X$ называется лагранжсевым, если $\left(T_{(x, \xi)} \Lambda\right)^{\sigma}=T_{(x, \xi)} \Lambda$, где

$$
\left(T_{(x, \xi)} \Lambda\right)^{\sigma}=\left\{p \in T_{(x, \xi)}\left(T^{*} X\right): \sigma(p, q)=0, \forall q \in T_{(x, \xi)} \Lambda\right\} .
$$

В частности, имеем $\operatorname{dim} \Lambda=n$. Многообразие $\Lambda \subset T^{*} X \backslash 0=\left\{(x, \xi) \in T^{*} X: \xi \neq 0\right\}$ назьвается коническим, если $(x, \xi) \in \Lambda$ влечет $(x, \tau \xi) \in \Lambda$ для всех $\tau>0$. Пусть $\Sigma \subset X$-гладкое $k$-мерное подмногообразиев $X$. Его конормальное расслоение в $T^{*} X$ определяется как

$$
N^{*} \Sigma=\left\{(x, \xi) \in T^{*} X: x \in \Sigma, \xi(\delta x)=0, \forall \delta x \in T_{x} \Sigma\right\} .
$$

Доказательства последующих утверждений можно найти в [12], [13], [24]. Мы следуем в основном [10]. 
УТВЕРЖДЕНИЕ 2.1.

(1) Пусть $\Lambda \subset T^{*} X \backslash 0$ - замкнутое $n$-мерное подмногообразие. Тогда $\Lambda$ - коническое ланранжево многообразие тогда и только тогда, когда форма $\alpha$ в (2.1) обнуляется на $\Lambda$.

(2) Конормальное расслоение $N^{*} \Sigma$ гладжого $k$-мерного подмногообразия $\Sigma$ в $X$ является коническим лагранжевым многообразием.

(3) Пусть $\Lambda \subset T^{*} X \backslash 0$ - коническое лагранжево многообразие, и пусть $D \pi_{(x, \xi)}: T_{(x, \xi)} \Lambda \rightarrow T_{x} X$ имеет постоянный ранг $k$ для всех $(x, \xi) \in \Lambda$. Тогда любая точка $(x, \xi) \in \Lambda$ имеет коническую окрестность $\Gamma$ такую, что

(a) $\Sigma=\pi(\Lambda \cap \Gamma)$ - гладкое $k$-мерное подмногообразие $X$;

(b) $\Lambda \cap \Gamma$ открыто в $N^{*} \Sigma$.

В дальнейшем мы будем в основном иметь дело с коническими лагранжевыми многообразиями, и нам потребуются выражения в локальных координатах. Для этого рассмотрим локальную тривиализацию $X \times\left(\mathbb{R}^{n} \backslash 0\right)$ расслоения $T^{*} X \backslash 0$ и будем предполагать, что $X$ - открытое множество размерности $n$. Однако, в дальнейшем нам также потребуется более обшая постановка, поэтому разрешим размерности пространства $X$ отличаться от размерности слоев. Рассмотрим конус $\Gamma$ в $X \times\left(\mathbb{R}^{N} \backslash 0\right)$. Гладкая функция $\phi: X \times\left(\mathbb{R}^{N} \backslash 0\right) \rightarrow \mathbb{R}$ называется фазовой функиией, если она однородна по $\theta$ с порядком 1 и не имеет критических точек по $(x, \theta): \phi(x, \tau \theta)=\tau \phi(x, \theta)$ для $\tau>0$ и $d_{(x, \theta)} \phi(x, \theta) \neq 0$ для всех $(x, \theta) \in X \times\left(\mathbb{R}^{N} \backslash 0\right)$. Фазовая функция называется невырожденной в Г, если для всех $(x, \theta) \in \Gamma$ с $d_{\theta} \phi(x, \theta)=0$ векторы $d_{(x, \theta)} \frac{\partial \phi(x, \theta)}{\partial \theta_{j}}$ линейно независимы, $j=1, \ldots, N$.

УТВЕРЖДЕНИЕ 2.2 .

(1) Для любого конуса $\Gamma$ в $X \times\left(\mathbb{R}^{N} \backslash 0\right)$ и невырожденной фазовой функции $\phi$ в Г существует открытый конус $\widetilde{\Gamma} \supset$ такой, что множество

$$
C_{\phi}=\left\{(x, \theta) \in \widetilde{\Gamma}: d_{\theta} \phi(x, \theta)=0\right\}
$$

- гладкое коническое $~ п$-мерное подмногообразие $X \times\left(\mathbb{R}^{N} \backslash 0\right)$. Отображение

$$
T^{\phi}: C_{\phi} \ni(x, \theta) \mapsto\left(x, d_{x} \phi(x, \theta)\right) \in T^{*} X \backslash 0
$$

задает погружение, коммутирующее с умножением на вещественные числа в слоях. Обозначим $\Lambda_{\phi}=T^{\phi}\left(C_{\phi}\right)$.

(2) n-мерное подмногообразие $\Lambda$ в $T^{*} X \backslash 0$ является коническим лагранжевым многообразием тогда и только тогда, когда любая точка $(x, \xi) \in \Lambda$ имеет коническую окрестность $\Gamma$ такую, что $\Lambda \cap \Gamma=\Lambda_{\phi}$ для некоторой невырожденной фазовой функиии ф.

Очевидно, условие, что $\Lambda$ - конус, соответствует положительной однородности с порядком 1 фазовой функции $\phi$. 
Пусть $\Lambda$ - замкнутое коническое лагранжево подмногообразие в $T^{*} X \backslash 0$. Обобшенная функция $u$ назьвается распределением Фурье порядка $\mathrm{m}$, ассоциированным $c \Lambda, u \in I^{m}(X, \Lambda)$, если

$$
\prod_{j=1}^{n} P_{j} u \in{ }^{\infty} H_{-m-n / 4}^{\mathrm{loc}}(X)
$$

где $P_{j} \in \Psi^{1}(X)$ - псевдодифференциальные операторы, чьи главные символы $p_{j}(x, \xi)$ равны нулю на $\Lambda$, и $^{\infty} H_{-m-n / 4}^{\text {loc }}$ локализация стандартных пространств Бесова. Обобщенная функция $u \in \mathscr{S}^{\prime}\left(\mathbb{R}^{n}\right)$ принадлежит пространству Бесова ${ }^{\infty} H_{\sigma}\left(\mathbb{R}^{n}\right)$, если $\widehat{u} \in L_{\text {loc }}^{2}\left(\mathbb{R}^{n}\right)$ и

$$
\|u\|_{\infty} H_{\sigma}\left(\mathbb{R}^{n}\right)=\left(\int_{|\xi| \leqslant 1}|\widehat{u}(\xi)|^{2} d \xi\right)^{1 / 2}+\sup _{j \geqslant 0}\left(\int_{2^{j} \leqslant|\xi| \leqslant 2^{j+1}}\left|2^{\sigma j} \widehat{u}(\xi)\right|^{2} d \xi\right)^{1 / 2}<\infty .
$$

Для гладкого $n$-мерного многообразия $X$ локальное пространство Бесова ${ }^{\infty} H_{\sigma}^{\text {loc }}(X)$ определено как пространство всех $u \in \mathscr{D}^{\prime}(X)$, для которых $(\psi u) \circ \varkappa^{-1}$ принадлежит ${ }^{\infty} H_{\sigma}\left(\mathbb{R}^{n}\right)$, где $\Omega \subset X$ - карта с системой координат $\varkappa$, и $\psi \in C_{0}^{\infty}(\Omega)$. Подробности см. в $[24 ; 25.1]$ и $[56 ; 6.1]$.

Пусть теперь $X, Y$ - открытые множества в $\mathbb{R}^{n}$, и пусть $\Phi$-невырожденная фазовая функция в $X \times Y \times \mathbb{R}^{N}$. Пусть $a \in S_{\rho}^{m}\left(X \times Y \times \mathbb{R}^{N}\right)$ - символ типа $\rho$ и порядка $m$, т.е. $a \in C^{\infty}\left(X \times Y \times \mathbb{R}^{N}\right), 1 / 2 \leqslant \rho \leqslant 1$, и для любого компактного множества $K$ в $X \times Y$ и любых мультииндексов $\alpha, \beta$ выполнено

$$
\left|\partial_{x, y}^{\beta} \partial_{\xi}^{\alpha} a(x, \theta)\right| \leqslant C(\alpha, \beta, K)(1+|\theta|)^{m-\rho|\alpha|+(1-\rho)|\beta|}
$$

для всех $(x, y) \in K$ и $\theta \in \mathbb{R}^{N} \backslash 0$. Интегральнылми операторами Фурье называются операторы $T$ типа

$$
T u(x)=\int_{Y} \int_{\mathbb{R}^{N}} e^{i \Phi(x, y, \theta)} a(x, y, \theta) u(y) d \theta d y .
$$

Выражение (2.3) можно интерпретировать в классическом смысле абсолютной сходимости интегралов для $m+N<0$ и $u \in C_{0}^{\infty}(X)$. Ядро Шварца оператора (2.3) задается с помошью обобшенной функции

$$
K(x, y)=\int_{\mathbb{R}^{N}} e^{i \Phi(x, y, \theta)} a(x, y, \theta) d \theta
$$

а в соответствии с утверждением 2.2 множество

$$
\Lambda_{\Phi}=\left\{\left(x, y, d_{x} \Phi(x, y, \theta), d_{y} \Phi(x, y, \theta)\right): d_{\theta} \Phi(x, y, \theta)=0\right\}
$$

- коническое лагранжево $2 n$-мерное подмногообразие в $T^{*}(X \times Y) \backslash 0$. При $\rho=1$ ядро Шварца (2.4) - распределение Фурье в $X \times Y$, ассоциированное с $\Lambda_{\Phi}$, порядка $\mu=m-n / 2+N / 2, K \in I^{\mu}\left(X \times Y, \Lambda_{\Phi}\right)$. Обратно, любое распределение Фурье $K \in I^{\mu}(X \times Y, \Lambda)$ микролокально записьвается в виде (2.4) с точностью до гладких 
слагаемых с $\rho=1$ (см. [56; теорема 6.1.4]). В обшем, пространство распределений Фурье $I_{\rho}^{\mu}(X, \Lambda)$ состоит из распределений Фурье

$$
u(x)=\int_{\mathbb{R}^{N}} e^{i \phi(x, \theta)} a(x, \theta) d \theta
$$

с $\Lambda$, локально имеющим вид $\Lambda_{\phi}$, как в утверждении $2.2, a \in S_{\rho}^{m}\left(X \times \mathbb{R}^{N}\right), \rho>1 / 2$, и $\mu=m-n / 4+N / 2$.

Интеграл (2.3) может зависеть не от всех переменных $\theta$, и порядок $T$ определяется как $\mu=m+(N-n) / 2$, т.е. как порядок его распределения Фурье (в этом случае имеем $X \times Y$ вместо $X)$. Следуюшая теорема описывает семейство фазовых функций, соответствующих одному и тому же распределению Фурье (2.4) (теорема 2.3 .4 в [10]). Сформулируем ее для обшего случая $I_{\rho}^{\mu}(X, \Lambda)(2.5)$ вместо специального случая $I_{\rho}^{\mu}(X \times Y, \Lambda)$.

TEOPEMA 2.3. Пусть $\phi(x, \theta)$ и $\widetilde{\phi}(x, \widetilde{\theta})$ - невырожсденные фазовые функиии в точках $\left(x_{0}, \theta_{0}\right) \in X \times\left(\mathbb{R}^{N} \backslash 0\right) u\left(x_{0}, \widetilde{\theta}_{0}\right) \in X \times\left(\mathbb{R}^{\tilde{N}} \backslash 0\right)$ соответственно. Пусть $\Gamma$ и $\widetilde{\Gamma}$ - открытые конические окрестности точек $\left(x_{0}, \theta_{0}\right)$ u $\left(x_{0}, \widetilde{\theta}_{0}\right)$ такие, что отображения $T_{\phi}: C_{\phi} \rightarrow \Gamma_{\phi}$ и $T_{\widetilde{\phi}}: C_{\widetilde{\phi}} \rightarrow \Gamma_{\widetilde{\phi}}$ coответственно ингективны. Если $\Lambda_{\phi}=\Lambda_{\tilde{\phi}}$, то любое распределение Фурье, задаваемое фазовой функичей $\phi и$ амплитудой $a \in S_{\rho}^{m}\left(X \times \mathbb{R}^{N}\right), \rho>1 / 2, c \operatorname{ess} \operatorname{supp} a$, содержашимся в достаточно малой конической окрестности точки $\left(x_{0}, \theta_{0}\right)$, равно распределению Фурье, задаваемому фазовой функиией $\widetilde{\phi}$ и амплитудой $\widetilde{a} \in S_{\rho}^{m+\frac{1}{2}(N-\widetilde{N})}\left(X \times \mathbb{R}^{\tilde{N}}\right)$.

В частности, фазовую функцию $\Phi$ оператора $T$ в 2.3 можно всегда записать в виде

$$
\Phi(x, y, \xi)=\langle x, \xi\rangle-\psi\langle y, \xi\rangle,
$$

с некоторой функцией $\psi$ и $\xi \in \mathbb{R}^{n}$, чем мы будем неоднократно пользоваться в дальнейшем. Функция $\psi$ (как и $\Phi$ ) также называется производящей функцией для $\Lambda_{\Phi}$.

Таким образом, понятие интегрального оператора Фурье становится независимым от выбора фазовой функции, ассоциированной с лагранжевым многообразием $\Lambda$. Множество

$$
C=\Lambda^{\prime}=\left\{((x, \xi),(y, \eta)) \in T^{*} X \times T^{*} Y:(x, y, \xi,-\eta) \in \Lambda\right\}
$$

- коническое лагранжево многообразие в $T^{*} X \backslash 0 \times T^{*} Y \backslash 0$ с симплектической формой $\sigma_{X} \oplus-\sigma_{Y} . C$ называется однородным каноническим отношением из $T^{*} Y$ в $T^{*} X$. Пространство интегральных операторов с ядрами Шварца в $I_{\rho}^{\mu}(X \times Y, \Lambda)$ будем обозначать через $I_{\rho}^{\mu}(X, Y ; C)$. Элементы этого пространства называются интегральными операторами Фурье, ассоциированными с каноническим отношением $C=\Lambda^{\prime}$.

2.2. Функциональные пространства. В этом разделе мы вкратце обсудим пространства, встречаюшиеся в статье. Пусть $X$ - гладкое многообразие с мерой $\lambda$. Для $1 \leqslant p<\infty$ через $L^{p}(X)$ обозначим стандартное пространство (классов эквивалентности) функций $f$ на $X$ с конечной нормой $\|f\|_{p}=\left(\int_{X}|f|^{p} d \lambda\right)^{1 / p}$. Для $0<p<1$ это выражение не является нормой, и в теории сингулярных интегральных операторов пространство $L^{p}(X)$ часто заменяется на пространство Харди $H^{p}(X)$. Общую теорию комплексных и вещественных пространств Харди см. в [58], [61], [16], [59], где 
можно найти доказательства последующих утверждений. Так как в настоящей статье мы заинтересованы в локальных свойствах операторов, мы рассмотрим только пространства $H^{p}\left(\mathbb{R}^{n}\right)$.

Пусть $\mathscr{S}$ - пространство Шварца гладких быстро убываюших функций, оснашенное счетным семейством полунорм

$$
\|\phi\|_{\alpha, \beta}=\sup _{x \in \mathbb{R}^{n}}\left|x^{\alpha} \partial_{x}^{\beta} \phi(x)\right| .
$$

Пусть $\Phi \in \mathscr{S}$, для $t>0$ определим $\Phi_{t}(x)=t^{-n} \Phi(x / t)$. Тогда для обобшенной функции $f$ свертки $f * \Phi_{t}$ гладки и можно определить максимальньй оператор

$$
M_{\Phi} f(x)=\sup _{t>0}\left|\left(f * \Phi_{t}\right)(x)\right| .
$$

Пусть $\mathscr{F}$ - конечное семейство полунорм на $\mathscr{S}$, определим $\mathscr{S}_{\mathscr{F}}$ как множество всех $\Phi \in \mathscr{S}$ таких, что $\|\Phi\|_{\alpha, \beta} \leqslant 1$ для всех $\|\cdot\|_{\alpha, \beta} \in \mathscr{F}$. Максимальный оператор, ассоциированньй с семейством $\mathscr{S}_{\mathscr{F}}$, можно определить как

$$
\mathscr{M}_{\mathscr{F}} f(x)=\sup _{\Phi \in \mathscr{S}_{\mathscr{F}}} M_{\Phi} f(x) .
$$

За определение пространства Харди $H^{p}\left(\mathbb{R}^{n}\right)$ возьмем одно из следующих эквивалентных условий.

УТВЕРЖДЕНИЕ 2.4. Для обобщенной функиии $f$ и для $0<p \leqslant \infty$ следующие условия әквивалентны:

(1) Существует функиия $\Phi \in \mathscr{S} c \int \Phi d x \neq 0$ такая, что $M_{\Phi} f \in L^{p}\left(\mathbb{R}^{n}\right)$.

(2) Существует семейство $\mathscr{F}$ такое, что $\mathscr{M}_{\mathscr{F}} f \in L^{p}\left(\mathbb{R}^{n}\right)$.

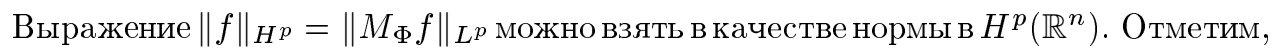
что это выражение эквивалентно $\left\|\mathscr{M}_{\mathscr{F}} f\right\|_{L^{p}}$ и является нормой только при $p \geqslant 1$. Для $0<p<1$ топологию пространства $H^{p}$ можно задать метрикой $d(f, g)=\|f-g\|_{H^{p}}^{p}$.

В случае $1<p \leqslant \infty$ легко показать, что $H^{p}\left(\mathbb{R}^{n}\right)$ совпадает с $L^{p}\left(\mathbb{R}^{n}\right)$. Однако, уже для $p=1$ имеем только $H^{1}\left(\mathbb{R}^{n}\right) \subset L^{1}\left(\mathbb{R}^{n}\right)$. С другой стороны, если $f \in L_{\text {comp }}^{1}\left(\mathbb{R}^{n}\right)$ удовлетворяет условию $\int f d x=0$ (что необходимо чтобы $f$ принадлежало $H^{1}\left(\mathbb{R}^{n}\right)$ ), то $f \in L^{q}\left(\mathbb{R}^{n}\right)$ для всех $q>1$ влечет $f \in H^{1}\left(\mathbb{R}^{n}\right)$. Часто используется декомпозищия функций из $H^{p}$ на атомы, похожая на классическую декомпозищию Кальдерона-Зигмунда. Для $0<p \leqslant 1$ атомами в $H^{p}$ называются функции, удовлетворяюшие следующим условиям:

(1) a сосредоточена в шаре $B$,

(2) $|a| \leqslant|B|^{-1 / p}$ почти всюду,

(3) $\int x^{\alpha} a(x) d x=0$ для всех $\alpha$ c $|\alpha| \leqslant n\left(p^{-1}-1\right)$.

Атомы из $H^{p}$ принадлежат $H^{p}\left(\mathbb{R}^{n}\right)$ с равномерной границей на норму $H^{p}$ и $L^{p}\left(\mathbb{R}^{n}\right)$ с $\int|a(x)|^{p} d x \leqslant 1$, что следует из свойств (1) и (2). 
УТВЕРЖДЕНИЕ 2.5. Пусть $0<p \leqslant 1$.

(1) Пусть $a_{k}$ - семейство атомов из $H^{p}$, и пусть $\lambda_{k} \in \mathbb{C}$ удовлетворяет $\sum_{k}\left|\lambda_{k}\right|^{p}<\infty$. Тогда ряд

$$
f=\sum_{k} \lambda_{k} a_{k}
$$

слабо сходится, его сумма $f$ принадлежит $H^{p}\left(\mathbb{R}^{n}\right) u$

$$
\|f\|_{H^{p}} \leqslant c\left(\sum_{k}\left|\lambda_{k}\right|^{p}\right)^{1 / p} .
$$

(2) Любую функиию $f \in H^{p}\left(\mathbb{R}^{n}\right)$ можсно представить в виде суммы ряда из атомов в $H^{p}$ как в (2.6). Ряд сходится в метрике $H^{p}$ и

$$
\left(\sum_{k}\left|\lambda_{k}\right|^{p}\right)^{1 / p} \leqslant c\|f\|_{H^{p}}
$$

Пусть $X$ - открытое множество в $\mathbb{R}^{n}$. Для $0<\gamma<1$ пространство Липшища (Гёльдера) $\operatorname{Lip}(X, \gamma)$ состоит из функций $f$, для которых существует константа $A$ такая, что $|f(x)| \leqslant A$ почти всюду и

$$
\sup _{x}|f(x-y)-f(x)| \leqslant A|y|^{\gamma}
$$

для всех $y$ с $x-y \in X$. Минимальную константу $A$, удовлетворяющую обоим неравенствам, можно взять в качестве нормы (см. [57], [65], [4]). Пространство Харди $H^{1}$ играет важную роль в методе комплексной интерполящии.

УТВЕРЖДЕНИЕ 2.6. Пусть $T_{z}$ - семейство линейных операторов в $\mathbb{R}^{n}$, параметризованное с помощвю $z$ с $0 \leqslant \operatorname{Re} z \leqslant 1$. Предположим, что для всех простых (ступенчатых) функиий $f, g$, сосредоточенных на множествах конечной меры, отображсение

$$
z \mapsto \int_{\mathbb{R}^{n}}\left(T_{z} f\right) g d x
$$

ограничено и аналитично в открытой полосе $0<\operatorname{Re} z<1$ и непрерывно в ее замыкании. Предположим, что $\left\|T_{z} f\right\|_{L^{1}} \leqslant C_{0}\|f\|_{H^{1}}$ nрu $\operatorname{Re} z=0 u\left\|T_{z} f\right\|_{q_{1}} \leqslant$ $C_{1}\|f\|_{p_{1}}$ при $\operatorname{Re} z=1$. Тогда имеем

$$
\left\|T_{t} f\right\|_{q_{t}} \leqslant C_{0}^{1-t} C_{1}^{t}\|f\|_{p_{t}}
$$

с $p_{t}$ и $q_{t}$, удовлетворяющими $1 / p_{t}=(1-t)+t / p_{1} u 1 / q_{t}=(1-t)+t / q_{1}$.

Доказательство основано на дуальности между $H^{1}$ и BMO ([16]). 
УТВеРЖДЕНИЕ 2.7 (Харди-Литтлвуд-Соболев). Для любъх $0<\gamma<n, 1<p<$ $q<\infty u 1 / q=1 / p-(n-\gamma) / n$ существует константа $A_{p q}$ такая, что

$$
\left\|f *\left(|y|^{-\gamma}\right)\right\|_{L^{q}} \leqslant A_{p q}\|f\|_{L^{p}} .
$$

Существует аналогичньй результат в пространствах Харди ([59; III.5.21]).

УТВЕРЖДЕНИЕ 2.8. Оператор $I_{\alpha} f=f *\left(|y|^{-\gamma}\right)$ допускает аналитическое продолжение на $-n\left(p^{-1}-1\right) \leqslant \operatorname{Re} \gamma<n$, если выполнено $\int x^{\alpha} f(x) d x=0$ при $|\alpha| \leqslant n\left(p^{-1}-1\right) u p \leqslant 1$. Для любих $0<p<q<\infty u 1 / q=1 / p-(n-\gamma) / n$ существует константа $A_{p q}$ такая, что

$$
\left\|f *\left(|y|^{-\gamma}\right)\right\|_{H^{q}} \leqslant A_{p q}\|f\|_{H^{p}} .
$$

Результат также справедлив для случаев $q=\infty, p \leqslant 1 u 0<p=q<\infty$, $\operatorname{Re} \gamma=n$.

Развитие теории комплексных пространств Харди можно проследить в [68] для $\mathbb{C}$ и в [11], [57], [61] для $\mathbb{C}^{n}$. Вешественную теорию с характеризацией в терминах максимальных операторов и декомпозиции Кальдерона-Зигмунда можно найти в [16]. Приложения пространств Харди к различным проблемам теории сингулярных интегральных операторов появились уже в [21]. Общую теорию и приложения можно найти в [57], [61] и [59].

2.3. Оценки. Пусть $X$ и $Y$ - гладкие паракомпактные вешественные $n$-мерные многообразия. Рассмотрим интегральный оператор Фурье $T \in I^{\mu}(X, Y ; \Lambda)$ с распределением Фурье порядка $\mu$ и волновым фронтом

$$
\Lambda^{\prime}=\{(x, \xi, y, \eta):(x, \xi, y,-\eta) \in \Lambda\} \subset T^{*} X \times T^{*} Y .
$$

Каноническое отношение $\Lambda$ является лагранжевым подмногообразием в $T^{*} X \times T^{*} Y$ с симплектической формой $\sigma_{X} \oplus-\sigma_{Y}$. Мы будем рассматривать операторы $T$, для которых каноническое отношение $\Lambda$ локально является графиком симплектоморфизма между $T^{*} X \backslash 0$ и $T^{*} Y \backslash 0$, оснашенными стандартными симплектическими формами $\sigma_{X}$ и $\sigma_{Y}$.

Пусть $\pi_{X \times Y}-$ стандартная проекция из $T^{*}(X \times Y)$ в $X \times Y$. По теореме о выборе фазовой функции, интегральное ядро оператора $T$ можно всегда привести к распределению Фурье вида

$$
K(x, y)=\int_{\mathbb{R}^{N}} e^{i \Phi(x, y, \theta)} a(x, y, \theta) d \theta .
$$

Символ $a$ оператора $T$ имеет порядок $m, a \in S^{m}$, где $m=\mu-(N-n) / 2$. По теореме о выборе фазовой функции (теорема 2.3) число $N$ частотных переменных может быть выбрано равным $n$, и в этом случае порядки оператора и его символа совпадают. Множество $\Lambda^{\prime}$ локально имеет вид

$$
\Lambda^{\prime}=\left\{\left(x, \nabla_{x} \Phi, y, \nabla_{y} \Phi\right): \nabla_{\theta} \Phi(x, y, \theta)=0\right\} .
$$

Отметим, что однородность канонического отношения влечет $\left.\operatorname{rank} d \pi_{X \times Y}\right|_{\Lambda} \leqslant 2 n-$ 1. Важным результатом о $L^{p}$-непрерывности операторов из $I^{\mu}$ является следующая теорема $([53])$. 
Tеорема 2.9. Пусть $\Lambda \subset T^{*} X \backslash 0 \times T^{*} Y \backslash 0$ - коническое лагранжево многообразие, локальный граф. Пусть $1<p<\infty u \mu \leqslant-(n-1)|1 / p-1 / 2|$. Тогда оператор $T \in I^{\mu}(X, Y ; \Lambda)$ непрерывен из $L_{\mathrm{comp}}^{p}$ в $L_{\mathrm{loc}}^{p}$. Порядок $\mu$ точен для әллиптических операторов $T$, если $\left.d \pi_{X \times Y}\right|_{\Lambda}$ имеет максимальный ранг, равньй $2 n-1$, хотя бъ в одной точке.

Однако, если $\left.d \pi_{X \times Y}\right|_{\Lambda}$ не достигает ранга $2 n-1$, то порядок $\mu$ не точен и зависит от особенностей проекции $\left.\pi_{X \times Y}\right|_{\Lambda}$. Оказьвается, что свойства непрерывности интегральных операторов Фурье в пространствах $L^{p}$ с $p \neq 2$ зависят от максимального ранга проекции $\pi_{X \times Y}$ на $\Lambda$. Важной составляющей является следующее условие гладкой факторизации для $\pi_{X \times Y}$. Предположим, что сушествует такое $k, 0 \leqslant k \leqslant n-1$, что для любого $\lambda_{0}=\left(x_{0}, \xi_{0}, y_{0}, \eta_{0}\right) \in \Lambda$ сушествуют коническая окрестность $U_{\lambda_{0}} \subset \Lambda$ точки $\lambda_{0}$ и гладкое однородное порядка 0 отображение $\pi_{\lambda_{0}}: \Lambda \cap U_{\lambda_{0}} \rightarrow \Lambda$ с постоянным рангом rank $d \pi_{\lambda_{0}}=n+k$ такие, что выполнено

$$
\pi_{X \times Y}=\pi_{X \times Y} \circ \pi_{\lambda_{0}} .
$$

При этом предположении операторы $T \in I_{\rho}^{\mu}(X, Y ; \Lambda)$ с $1 / 2 \leqslant \rho \leqslant 1$ обладают лучшими свойствами непрерывности, чем описанные в теореме 2.9 .

TеOPема 2.10. Пусть $\Lambda \subset T^{*} X \backslash 0 \times T^{*} Y \backslash 0$ - коническое лагранжево многообразие, удовлетворяющее условию локального графика. Пусть $\mu \leqslant-(k+(n-k) \times$ $(1-\rho))|1 / p-1 / 2| c 1<p<\infty u 1 / 2 \leqslant \rho \leqslant 1$. Предположим, что условие гладкой факторизачии (2.9) выполнено. Тогда операторы $T \in I_{\rho}^{\mu}(X, Y ; \Lambda)$ непрерывны из $L_{\text {comp }}^{p}$ i $L_{\mathrm{loc}}^{p}$.

Отметим, что сушественная однородность символа $а$ здесь не сушественна. При $m=\mu-(N-n) / 2$ получаем $a \in S_{\rho}^{m}$, т.е.

$$
\left|\partial_{x, y}^{\alpha} \partial_{\theta}^{\beta} a(x, y, \theta)\right| \leqslant C_{\alpha \beta}(1+|\theta|)^{m-\rho|\beta|+(1-\rho)|\alpha|} .
$$

Результаты в $L^{2}$ для таких операторов можно найти в [24] при $1 / 2<\rho$ и в [18] при $\rho=1 / 2$. Операторы $T \in I_{\rho}^{0}$ непрерывны в $L^{2}$, и этот результат не зависит от условия (2.9). Доказательства теорем 2.9 и 2.10 основаны на применении метода комплексной интерполящии (как в утверждении 2.6) к непрерьвности в $L^{2}$ и в пространстве Харди $H^{1}$, для которого выполнена

ТЕОРема 2.11. Пусть А удовлетворяет условиям теоремы 2.10, и пусть число $N$ частотных переменных равно $n$. Пусть интегральный оператор Фурье $T$ имеет ядро Шварца $K$, заданное распределением Фурье (2.8). Предположим, что символ $a \in S_{\rho}^{k / 2+(n-k)(1-\rho) / 2}$ в (2.8) сосредоточен в компактном множестве в $\mathbb{R}_{x}^{n} \times \mathbb{R}_{y}^{n}$. Тогда оператор $T$ непрерьвен из $H^{1}\left(\mathbb{R}^{n}\right)$ в $L^{1}\left(\mathbb{R}^{n}\right)$.

Опишем вкратце доказательство приведенных теорем. Начнем с теорем 2.9 и 2.10 . Пусть $a$ - символ порядка $-(n-1)|1 / p-1 / 2|$, и предположим, что $1<p \leqslant 2$. Рас-

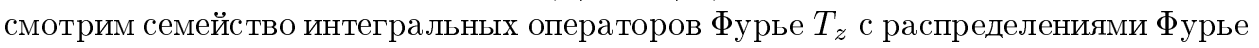

$$
K_{z}(x, y)=\int_{\mathbb{R}^{n}} e^{i \Phi(x, y, \theta)} a(x, y, \theta)\left(1+|\theta|^{2}\right)^{\frac{1}{2}[(n-1)(1 / p-1 / 2)-(n-1) / 2+z(n-1) / 2]} d \theta .
$$


Из непрерьвности операторов нулевого порядка в $L^{2}$ следует

$$
\left\|T_{z} f\right\|_{L^{2}} \leqslant C_{z}\|f\|_{L^{2}}, \quad \operatorname{Re} z=1
$$

и из теоремы 2.11 следует

$$
\left\|T_{z} f\right\|_{L^{1}} \leqslant C_{z}\|f\|_{H^{1}}, \quad \operatorname{Re} z=0
$$

Константы $C_{z}$ зависят от конечного числа производных символа и растут как полиномы по $|z|$. Применим утверждение $2.6 \mathrm{c} t=2(1-1 / p)$. Имеем $1 / p_{t}=1 / q_{t}=1-t / 2$, $(n-1)(1 / p-1 / 2)-(n-1) / 2+t(n-1) / 2=0$, так что $T_{t}=T$ и теорема 2.9 следует из утверждения 2.6. Аналогично, теорема 2.10 следует из теоремы 2.11 , если взять $a \in S_{\rho}^{-(k+(n-k)(1-\rho))|1 / p-1 / 2|}$ и ядро

$$
\begin{aligned}
K_{z}(x, y)= & \int_{\mathbb{R}^{n}} e^{i \Phi(x, y, \theta)} a(x, y, \theta) \\
& \quad \times\left(1+|\theta|^{2}\right)^{\frac{1}{2}[-(k+(n-k)(1-\rho))|1 / p-1 / 2|+(z-1)[k+(n-k)(1-\rho)] / 2]} d \theta .
\end{aligned}
$$

Для доказательства теоремы 2.11 воспользуемся разложением на атомы $f=\sum_{k} \lambda_{k} a_{k}$, как в утверждении 2.5. Если шар $B$ из определения атома содержит единичный куб, то по неравенству Коши-Шварца имеем:

$$
\left\|T a_{k}\right\|_{L^{1}} \leqslant C\left|\operatorname{supp} a_{k}\right|^{1 / 2}\left\|T a_{k}\right\|_{L^{2}} \leqslant C^{\prime}\left|\operatorname{supp} a_{k}\right|^{1 / 2}\left\|a_{k}\right\|_{L^{2}},
$$

что равномерно ограничено по свойству (2) в определении атомов. Таким образом, в виду утверждения 2.5 теорема 2.11 сводится к равномерной оценке $\left\|T a_{k}\right\|_{L^{1}} \leqslant C$ для всех атомов, сосредоточенных в достаточно малых шарах. Такая оценка получается с помошью конического разбиения фазового пространства, где к каждому конусу в свою очередь применяется диадическое разбиение. Применяя соответствующее разбиение единицы, в каждой полученной области можно заменить фазовую функцию оператора $T$ на линейную функцию, для которой интегрированием по частям получаются оценки в $L^{1}$. Оказывается, что ошибка, возникающая при этой замене, суммируема, откуда вытекает теорема 2.11. Подробное доказательство можно найти в [53].

В разделе 7 мы обсудим точность результата теоремы 2.10. В частности, с помошњ метода стационарной фазы мы покажем, что при $\rho=1$ порядок $\mu$ в теореме 2.10 точен для эллиптических операторов $T$, для которых ранг $n+k$ достигается хотя бы в одной точке. В разделе 2.4 мы покажем, что факторизация $\left.\pi_{X \times Y}\right|_{\Lambda}$ эквивалентна факторизации гессиана фазовой функции оператора. В свою очередь это условие ведет к параметрическому уравнению Монжа-Ампера (см. [28]), что мы обсудим в разделе 6.2.

Для получения оценок из $L^{p}$ в $L^{q}$ опять используется метод комплексной интерполяции (утверждение 2.6). Пусть $a \in S^{\mu}\left(\mathbb{R}^{n} \times \mathbb{R}^{n} \times \mathbb{R}^{n}\right)$, и пусть $\Phi(x, y, \xi)$ - невырожденная фазовая функция. Пусть интегральньй оператор Фурье микролокально задан формулой

$$
T f(x)=\int_{\mathbb{R}^{n}} \int_{\mathbb{R}^{n}} e^{i \Phi(x, y, \xi)} a(x, y, \xi) f(y) d y d \xi .
$$


Tеорема 2.12. Пусть $T \in I^{\mu} u 1<p, q<\infty$. Тогда имеем:

(1) Если $p \leqslant 2 \leqslant q$, то оператор $T: L^{p}\left(\mathbb{R}^{n}\right) \rightarrow L^{q}\left(\mathbb{R}^{n}\right)$ непрерьвен при $\mu \leqslant n(1 / q-1 / p)$.

(2) Если $1<p \leqslant q \leqslant 2$, то оператор $T: L^{p}\left(\mathbb{R}^{n}\right) \rightarrow L^{q}\left(\mathbb{R}^{n}\right)$ непрерывен при $\mu \leqslant 1 / q-n / p+(n-1) / 2$. Двойственньй результат выполнен при $2 \leqslant p \leqslant q<\infty$.

(3) При $p=1$ утверждения пn. (1) и (2) выполнень, если $L^{1}\left(\mathbb{R}^{n}\right)$ заменить пространством Харди $H^{1}\left(\mathbb{R}^{n}\right)$.

ЗАмечАниЕ 2.13. В случае $\operatorname{rank} D_{\xi \xi}^{2} \Phi=n-1$ утверждение теоремы $2.12,(1)$, можно усилить. Пусть $1<p, q<\infty$, через $p^{\prime}, q^{\prime}$ обозначим их сопряженные индексы. Тогда оператор $T$ непрерьвен из $L^{p}\left(\mathbb{R}^{n}\right)$ в $L^{q}\left(\mathbb{R}^{n}\right)$, если

$$
\begin{aligned}
& \mu \leqslant \frac{1}{q}-\frac{n}{p}+\frac{n-1}{2}, \quad q \leqslant p^{\prime}, \\
& \mu \leqslant \frac{n}{q}-\frac{1}{p}-\frac{n-1}{2}, \quad q \geqslant p^{\prime} .
\end{aligned}
$$

Утверждение распространяется на случай $p=1$, если $L^{1}\left(\mathbb{R}^{n}\right)$ заменить пространством Харди $H^{1}\left(\mathbb{R}^{n}\right)$.

Доказательство замечания 2.13 следует по методу комплексной интерполящии между теоремой 2.9 и тем фактом, что при условиях замечания 2.13 оператор $T$ непрерьвен из $H^{1}\left(\mathbb{R}^{n}\right)$ в $L^{\infty}\left(\mathbb{R}^{n}\right)$ при $\mu=-(n+1) / 2$. Оптимальность границ для $\mu$ в замечании 2.13 показана в [59; IX.6.16] для операторов с фазовой функцией $\Phi(x, y, \xi)=\langle x-y, \xi\rangle+|\xi|$. См. также раздел 7 .

ЗАмЕчАниЕ 2.14. Из утверждения 2.8 с $p=1$ и $q=2$ следует, что операторы порядка $-n / 2$ непрерывны из $H^{1}$ в $L^{2}$.

УТВЕРЖДЕНИЕ 2.15. При выполнении условия факторизачии (2.9) и при $1<$ $p \leqslant q \leqslant 2$ или $2 \leqslant p \leqslant q<\infty u \mu \leqslant-n / p+(n-k) / q+k / 2$ onepamopb $T \in$ $I^{\mu}(X, Y ; \Lambda)$ непрерывны из $L_{\mathrm{comp}}^{p}(Y)$ в $L_{\mathrm{loc}}^{q}(X)$.

Доказательство следует из интерполяции между утверждениями теоремы 2.10 и замечания 2.14. Аналогично можно получить, что операторы из $I^{\mu}$ непрерьвны из $\operatorname{Lip}(\alpha-k / 2-\mu)$ в $\operatorname{Lip}(\alpha)$ для всех $\alpha \in \mathbb{R}$. Получаем следствие для псевдодифференциальных операторов.

УтвеРЖДЕнИе 2.16. Пусть $P \in \Psi^{\mu}(X) u 1<p \leqslant q<\infty$. Тогда $P: L_{\text {comp }}^{p}(X) \rightarrow$ $L_{\mathrm{loc}}^{q}(X)$ непрерывен при $\mu \leqslant-n(1 / p-1 / q)$.

ДокАЗАТЕльСТво. Приведем прямое доказательство без использования интерполяционных методов. Случай $p=q$ хорошо известен и следует, например, из теоремы 2.10 с $k=0$ и $\mu \leqslant 0$. Предположим, что $p<q$. Оператор $(I-\Delta)^{-\mu / 2} \circ P$ непрерывен в $L^{p}$, и утверждение сводится к свойствам оператора $(I-\Delta)^{\mu / 2}$. Его главньй символ однороден с порядком $\mu$, и его интегральное ядро имеет порядок $-n-\mu$ в $|x-y|$. Таким образом, оператор $(I-\Delta)^{\mu / 2}$ имеет вид

$$
I_{\gamma} f(x)=\int_{\mathbb{R}^{n}}|x-y|^{-\gamma} f(y) d y
$$


с $\gamma=n+\mu$. Для $\mu<0$ из из утверждения 2.7 следует что $I_{\gamma}$ непрерывен из $L^{p}$ в $L^{q}$, если $1 / q=1 / p+\mu / n$, что завершает доказательство утверждения 2.16.

Под аналитическим интегральныцм оператором фурье мы будем понимать оператор, действуюший в вешественных аналитических многообразиях $X$ и $Y$, чье каноническое отношение аналитично. Для таких операторов фазовая функция вешественно аналитична и условие гладкой факторизации можно продолжить в комплексную область. Полученньй анализ можно применить к непрерывности в $L^{p}$ операторов на $\mathbb{R}^{n}$ c $n \leqslant 4$ и на $\mathbb{R}^{n}$ с произвольным $n$ и условием $\left.\operatorname{rank} d \pi_{X \times Y}\right|_{\Lambda} \leqslant n+2$, что описано в разделе 5 .

2.4. Параметрические слоения. Сразу сведем обшую постановку к открытым множествам в $\mathbb{R}^{n}$. Это не ограничивает общности, так как мы заинтересованы в локальных свойствах операторов. Заметим, что множества $X$ и $Y$ должны быть одинаковой размерности, что следует из условия, что каноническое отношение - локальный график. В общем, каноническое отношение интегрального оператора Фурье в $T^{*} X \times T^{*} Y$ можно рассматривать как гладкое семейство лагранжевых многообразий в $T^{*} \mathbb{R}^{n}$, параметризованных точками из $T^{*} \mathbb{R}^{n}$. Вначале покажем, что при таком представлении ранги проекций на базу отличаются на $n$. По теореме о выборе производящей функции (теорема 2.3) можно показать, что однородное каноническое отношение имеет вид

$$
\Lambda=\left\{\left(\nabla_{\xi} \phi(y, \xi), \xi, y, \nabla_{y} \phi(y, \xi)\right)\right\}
$$

в окрестности точки $\left(x_{0}, \xi_{0}, y_{0}, \eta_{0}\right)$. Производяшая функция $\phi$ удовлетворяет условию

$$
\nabla_{\xi} \phi\left(y_{0}, \xi_{0}\right)=x_{0}, \quad \nabla_{y} \phi\left(y_{0}, \xi_{0}\right)=\eta_{0} .
$$

Фазовая функция оператора $T \in I_{\rho}^{\mu}(X, Y ; \Lambda)$ имеет вид

$$
\Phi(x, y, \xi)=\langle x, \xi\rangle-\phi(y, \xi),
$$

и $\Lambda=\Lambda_{\Phi}$ локально задано как множество $\left\{\left(\nabla_{\xi} \phi(y, \xi), \xi, y, \nabla_{y} \phi(y, \xi)\right)\right\}$. Мы будем часто обозначать через $\Lambda_{\Phi}$ множество типа $(2.10)$ с фазовой функцией $\Phi$ как в $(2.11)$. Микролокально ядро Шварца (2.8) оператора $T$ принимает вид

$$
K(x, y)=\int_{\mathbb{R}^{n}} e^{i(\langle x, \xi\rangle-\phi(y, \xi))} b(x, y, \xi) d \xi
$$

с некоторым символом $b \in S_{\rho}^{\mu}$. Таким образом, локально ядра Шварца операторов из $I_{\rho}^{\mu}(X, Y ; \Lambda)$ с точностью до гладкого слагаемого представляют собой конечную сумму ядер вида (2.12) с символами $b \in S_{\rho}^{\mu}$, каждый из которых может быть записан в своей системе координат.

Таким образом, мы будем полагать, что $X$ и $Y$ - открытые множества в $\mathbb{R}^{n}$. Условие локального графика равносильно условию

$$
\operatorname{det} \phi_{y \xi}^{\prime \prime}(y, \xi) \neq 0
$$


на носителе символа оператора $T$. В этой локальной системе координат проекция $\left.\pi_{X \times Y}\right|_{\Lambda}$ принимает вид

$$
\left.\pi_{X \times Y}\right|_{\Lambda}:\left(\nabla_{\xi} \phi(y, \xi), \xi, y, \nabla_{y} \phi(y, \xi)\right) \mapsto\left(\nabla_{\xi} \phi(y, \xi), y\right)
$$

Уровни проекции $\left.\pi_{X \times Y}\right|_{\Lambda}$ можно параметризовать точками $\xi$, для которых градиент $\nabla_{\xi} \phi(y, \xi)$ постоянен. С другой стороны, в силу представления открытого подмножества $\Lambda_{0}$ канонического отношения $\Lambda$ в виде конормального расслоения $N^{*} \Sigma_{0}$ гладкого подмножества $\Sigma_{0}$ множества $\Sigma=\pi_{X \times Y}(\Lambda)$ (утверждение 2.1$)$ уровни $\left.\pi_{X \times Y}\right|_{\Lambda}$ в $\Lambda_{0}$ в точке $\left(x_{0}, \xi_{0}, y_{0}, \eta_{0}\right)$ - линейные подпространства в $T_{\left(x_{0}, y_{0}\right)}^{*}(X \times Y)$. По $(2.14)$ эти линейные подпространства заданыпарами $\left(\xi, \nabla_{y} \phi(y, \xi)\right)$. Следовательно, множество точек $\xi$ в уровне $\nabla_{\xi} \phi(y, \xi)$ - линейное подпространство частотного $n$-мерного пространства $\Xi$. Несложно получить зависимость этого подпространства от $(y, \xi)$ с помощью следующего утверждения.

Лемма 2.17. Отображсение $\gamma: Y \times \Xi \rightarrow T^{*} X \times T^{*} Y$, определенное с помощью равенства

$$
\gamma(y, \xi)=\left(\nabla_{\xi} \phi(y, \xi), \xi, y, \nabla_{y} \phi(y, \xi)\right)
$$

задает диффеоморфизм между $Y \times \Xi и \Lambda$. Для каждого $y \in Y$ сужение $\xi \mapsto$ $\gamma(y, \xi)$ задает диффеоморфизм из $\Xi$ в $\Lambda \cap\left(\mathbb{R}^{n} \times \mathbb{R}^{n} \times\{y\} \times \mathbb{R}^{n}\right)$, его обращение является проекиией $(x, \xi, y, \eta) \mapsto \xi$.

Таким образом, отображение $\left.d \pi_{X \times Y}\right|_{\Lambda}$ изоморфно $\left.d \pi_{X \times Y} \circ d \gamma\right|_{Y \times \Xi}$, и, в частности, их ядра изоморфны. В то же время имеем

$$
\left.\operatorname{ker} d \pi_{X \times Y} \circ d \gamma\right|_{Y \times \Xi}(y, \xi)=\left\{(\delta y, \delta \xi): \frac{\partial^{2} \phi}{\partial \xi^{2}}(y, \xi) \delta \xi+\frac{\partial^{2} \phi}{\partial y \partial \xi}(y, \xi) \delta y=0, \delta y=0\right\}
$$

Получаем

$$
\left.\operatorname{ker} d \pi_{X \times Y} \circ d \gamma\right|_{Y \times \Xi}(y, \xi)=\left(0, \operatorname{ker} \frac{\partial^{2} \phi}{\partial \xi^{2}}(y, \xi)\right)
$$

Мы доказали следующую характеризацию проекции в терминах фазовой функции.

ТЕОрема 2.18. Пусть локальный график $\Lambda$ задан с помощью фазовой функиии $\Phi(x, y, \xi)=\langle x, \xi\rangle-\phi(y, \xi)$. Тогда для каждого $0 \leqslant k \leqslant n-1$ следующие условия әквивалентны:

(1) $\left.\operatorname{rank} d \pi_{X \times Y}\right|_{\Lambda_{\Phi}} \leqslant n+k$;

(2) $\left.\operatorname{rank} d \pi_{X \times Y} \circ d \gamma\right|_{\{y\} \times \Xi} \leqslant k$ для всех $y \in Y$ с $\gamma$ из лемми 2.17;

(3) $\operatorname{rank} \frac{\partial^{2} \phi}{\partial \xi^{2}}(y, \xi) \leqslant k$ для всех $y \in Y$ u $\xi \in \Xi$. 
ЗАМЕчАНИЕ 2.19. Из линейности уровней проекции $\left.\pi_{X \times Y}\right|_{\Lambda}$ следует, что для любого $\lambda \in \Lambda$ имеем локальное равенство

$$
\pi_{X \times Y}^{-1}\left(\pi_{X \times Y}(\lambda)\right) \cap \Lambda=\left.\operatorname{ker} d \pi_{X \times Y}\right|_{\Lambda}(\lambda) .
$$

С другой стороны, используя линейность уровней отображения $\nabla_{\xi} \phi(y, \xi)$ и изоморфизм в (2.15), получаем, что уровни отображения

$$
\xi \mapsto \nabla_{\xi} \phi(y, \xi)
$$

заданы ядрами матрищы $D_{\xi \xi}^{2} \phi(y, \xi)$. Это утверждение также следует, если применить рассуждения раздела 4 напрямую к функции $\phi$.

Уровни $\left.\pi_{X \times Y}\right|_{\Lambda}$ соответствуют уровням по $\xi$ градиента $\nabla_{\xi} \phi(y, \xi)$. В частности, они не пересекаются и продолжимы в некоторое открытое множество $\Omega$ в $\mathbb{C}^{n}$, если $\phi$ аналитична. Таким образом, получаем слоения в $\Omega \cap \mathbb{R}^{n}$ и $\Omega$ уровнями по $\xi$ градиента $\nabla_{\xi} \phi(y, \xi)$ и его голоморфного продолжения.

ЗАмЕчАниЕ 2.20. Если максимальньй ранг отображения $\left.d \pi_{X \times Y}\right|_{\Lambda}$ равен $n+k$, обозначим через $\Lambda^{(k)}$ множество, на котором он достигается. В терминах слоений слоение линейными пространствами в замечании 2.19 задается гладким отображением

$$
\varkappa_{\mathbb{R}}: \gamma^{-1}\left(\Lambda^{(k)}\right) \ni(y, \xi) \mapsto \operatorname{ker} \frac{\partial^{2} \phi}{\partial \xi^{2}}(y, \xi) \in \mathbb{G}_{n-k}\left(\mathbb{R}^{n}\right) .
$$

Условие факторизации (2.9) эквивалентно условию, что отображение $\varkappa_{\mathbb{R}} \circ \gamma^{-1}$ локально гладко продолжимо с $\Lambda^{(k)}$ на $\Lambda$. Продолжение соответствует слоению ядрами $\operatorname{ker} d \pi_{\lambda_{0}}$ с $\pi_{\lambda_{0}}$ из (2.9). В терминах слоений множество существенных особенностей отображения $\varkappa_{\mathbb{R}}$ равно $\Omega^{\operatorname{sing}} \cap\left(\mathbb{R}^{n} \times \mathbb{R}^{n}\right)$, где $\Omega^{\operatorname{sing}}$ - множество сушественных особенностей комплексного расширения отображения $\varkappa_{\mathbb{R}}$.

Следующий пример показывает, что условие факторизации не тривиально. В соответствии с замечаниями 2.19 и 2.20 по теореме 2.18 условие факторизации сводится к изучению условия $\operatorname{ker} \phi_{\xi \xi}^{\prime \prime}(y, \xi) \leqslant k$. В этом случае $\left.\operatorname{rank} d \pi_{X \times Y}\right|_{\Lambda_{\Phi}} \leqslant n+k$ по теореме $2.18,(3)$. Функция

$$
\phi(y, \xi)=\langle y, \xi\rangle+\frac{1}{\xi_{n}} \sum_{i=2}^{k+1}\left(y_{1} \xi_{1}+y_{i} \xi_{i}\right)^{2}
$$

удовлетворяет требуемому условию на ранг в окрестности точки $\xi_{n}=1$. С другой стороны, имеем

$$
\nabla_{\xi} \phi(y, \xi)=y+\frac{2}{\xi_{n}}\left(\begin{array}{c}
y_{1} \sum_{i=2}^{k+1}\left(y_{1} \xi_{1}+y_{i} \xi_{i}\right) \\
y_{2}\left(y_{1} \xi_{1}+y_{2} \xi_{2}\right) \\
\vdots \\
y_{k-1}\left(y_{1} \xi_{1}+y_{k-1} \xi_{k-1}\right) \\
0 \\
\vdots \\
0 \\
\frac{1}{\xi_{n}} \sum_{i=2}^{k+1}\left(y_{1} \xi_{1}+y_{i} \xi_{i}\right)^{2}
\end{array}\right)
$$


и для точек $y$ с $y_{i} \neq 0,1 \leqslant i \leqslant k+1$, уровни в (2.17) соответствуют уровням отображения $y_{1} \xi_{1}+y_{i} \xi_{i}, 2 \leqslant i \leqslant k+1$. Направление слоев в этом случае задано дробями $y_{i} / y_{1}, 2 \leqslant i \leqslant k+1$, которые не непрерьвны в $y=0$. Получаем

ПримеР 2.21. Пусть $1 \leqslant k \leqslant n-2$ и $x, y, \xi \in \mathbb{R}^{n}$. Функция

$$
\Phi(x, y, \xi)=\langle x-y, \xi\rangle-\frac{1}{\xi_{n}} \sum_{i=2}^{k+1}\left(y_{1} \xi_{1}+y_{i} \xi_{i}\right)^{2}
$$

удовлетворяет условию

$$
\left.\operatorname{rank} d \pi_{X \times Y}\right|_{\Lambda_{\Phi}} \leqslant n+k
$$

и задает локальный график $\Lambda_{\Phi}$, для которого слоение уровнями отображения $\left.\pi_{X \times Y}\right|_{\Lambda_{\Phi}}$ не имеет непрерьвного продолжения через $y=0$.

ЗАмечАниЕ 2.22 . В случае $k=0$ имеем конормальные операторы, которые можно свести к псевдодифференшиальным операторам композицией с интегральньм оператором Фурье, индуцированным гладкой заменой координат (см. раздел 7.2). Для таких операторов условие факторизации тривиально вьполнено. Случай $k=n-1$ соответствует условию $\left.\operatorname{rank} d \pi_{X \times Y}\right|_{\Lambda} \leqslant 2 n-1$, для которого условие факторизации выполнено благодаря однородности $\Lambda$. В этом случае можно взять $\pi_{\lambda_{0}}$ в $(2.9)$ как проекцию в коническом направлении.

ЗАМЕчАнИЕ 2.23. Из нарушения условия факторизации следует, что сингулярный носитель $\Sigma$ оператора $T$ не может быть гладким многообразием. В самом деле, если $\Sigma$ - гладкое многообразие, то $\Lambda=N^{*} \Sigma$ и условие факторизации вьполнено. Однако, наоборот, отсутствие гладкости у $\Sigma$ не влечет нарушение условия гладкой факторизации. Например, возьмем полукубическую параболу $G$ в $\mathbb{R}^{3}$ :

$$
G=\left\{x \in \mathbb{R}^{3}: x_{1}^{2}=x_{2}^{3}, x_{3}=0\right\}
$$

и зададим $\Sigma$ с помощью $\Sigma=\left\{(x, y) \in \mathbb{R}^{6}: x-y \in G\right\}$. Несложно убедиться, что соответствуюшая фазовая функция $\Phi$ имеет вид

$$
\Phi(x, y, \xi)=\langle x-y, \xi\rangle-\frac{4}{27} \frac{\xi_{2}^{3}}{\xi_{1}^{2}} .
$$

Очевидно, $\Sigma$ не гладко в нуле, в то время как условие факторизации выполнено для $\Phi$, что станет ясно в разделе 5.3 .

В разделе 4 мы обсудим вещественные слоения в классах $C^{m}$. Пока приведем следуюший пример.

ПримеР 2.24. Для $k \in \mathbb{N}$ функция

$$
\Phi(x, y, \xi)=\langle x-y, \xi\rangle-\frac{1}{\xi_{n}}\left(y_{1}^{k+1} y_{2}^{k+1} \xi_{1}+\left(y_{1}^{2}+y_{2}^{2}\right) \xi_{2}\right)^{2}, \quad y \in \mathbb{R}^{n}, \quad \xi \in \mathbb{R}^{n},
$$

удовлетворяет условию $\operatorname{rank} D_{\xi}^{2} \Phi \leqslant 1$ и задает локальный график, для которого слоение уровнями $\nabla_{\xi}$ Ф непрерывно продолжимо через $y=0$. Более того, локально это продолжение лежит в $C^{2 k-1}$, но не в $C^{2 k}$. 


\section{3. Аффинные слоения}

3.1. Методы комплексной аналитической геометрии. В этом разделе мы напомним некоторые понятия и факты комплексной аналитической геометрии, которые будут часто использоваться в статье. Следуя [46], введем

ОПРЕДЕЛЕНИЕ 3.1. Отображение $\tau: X \rightarrow Y$ между комплексными многообразиями $X$ и $Y$ называется мероморфным. если выполнены следующие три свойства:

(1) для любого $x \in X$ образ $\tau(x) \subset Y$ непуст и компактен в $Y$;

(2) график отображения $\tau$, т.е. множество пар $(x, y) \in X \times Y$ с $y \in \tau(x),-$ связное комплексно аналитическое подмножество в $X \times Y$ размерности, равной размерности $X$;

(3) сушествует плотное множество $X^{*}$ в $X$ такое, что для всех $x \in X^{*}$ образ $\tau(x)$ состоит только из одной точки.

Одним из важных методов для анализа структуры аналитических множеств являются оценки их размерности. Размерность произвольного подмножества $E$ комплексного $n$-мерного многообразия $M$ определим как

$$
\operatorname{dim} E=\sup \{\operatorname{dim} F: F \subset E\},
$$

где супремум берется по всем гладким подмногообразиям $F$ многообразия $M$, содержашимся в $E$. Если множество справа пусто, будем считать, что супремум равен $-\infty$. Если $E$ - подмногообразие или аналитическое подмножество многообразия $M$, то это определение размерности совпадает со стандартным. Если $\pi$ - проекция с декартова произведения на один из множителей, то верхняя граница на размерность прообраза дается с помошью следуюшей теоремы.

Tеорема 3.2. Пусть $E \subset M \times N$, где $M, N$ - комплексньее многообразия. Пусть $\pi: E \rightarrow M$ - естественная проекция. Предположим, что для $k \in \mathbb{N}$ виполнено

$$
\operatorname{dim} \pi^{-1}(z) \leqslant k, \quad \forall z \in \pi(E) .
$$

Tогда $\operatorname{dim} E \leqslant k+\operatorname{dim} \pi(E)$.

Глобально аналитическим подмножеством многообразия $M$ назьваются множества вида

$$
Z\left(f_{1}, \ldots, f_{k}\right)=\left\{z \in M: f_{1}(z)=\cdots=f_{k}(z)=0\right\},
$$

где $f_{1}, \ldots, f_{k}$ - голоморфные функции на $M$. Подмножество $Z$ многообразия $M$ назьвается аналитическим, если каждая точка в $M$ имеет открытую окрестность $U$ такую, что множество $Z \cap U$ - глобально аналитическое в $U$. Аналитические подмножества открытых множеств в $M$ называются локально аналитическими в $M$. В частности, множество $Z$ аналитично в $M$ тогда и только тогда, когда оно локально аналитично и замкнуто. 
УТВЕРЖДЕНИЕ 3.3.

(1) Пусть $V \subset M u W \subset N$ - непустье подмножества многообразий $M u$ $N$ соответственно. Тогда множество $V \times W$ (локально) аналитично в $M \times N$ тогда и только тогда, когда оба $V$ и $W$ (локально) аналитичньц в $M$ и $N$ соответственно.

(2) (теорема об аналитическом графике) Любое непрерьвное отображение $f: M \rightarrow N$ с аналитическим графиком голоморфно.

(3) Любое собственное аналитическое множество $Z$ в связном многообразии $M$ нигде не плотно, и его дополнение $M \backslash Z$ открыто и связно.

Если $V \subset M$ и $W \subset N$ - локально аналитические подмножества, то отображение $f: V \rightarrow W$ голоморфно, если каждая точка в $V$ имеет открытую окрестность $U$ в $M$ такую, что $\left.f\right|_{U \cap V}-$ сужение голоморфного отображения из $U$ в $N$.

Tеорема 3.4. Пусть $k \in \mathbb{N} u f$ - голоморфное отображсение, определенное выце. Тогда

(1) $\operatorname{dim} V \geqslant k+\operatorname{dim} f(V)$, ecлu $\operatorname{dim} f^{-1}(w) \geqslant k$ для $w \in f(V)$;

(2) $\operatorname{dim} V \leqslant k+\operatorname{dim} f(V)$, ecлu $\operatorname{dim} f^{-1}(w) \leqslant k$ для $w \in f(V)$.

Доказательство первой части основано на сведении оценки к подмножеству $Z$ образа $f(V)$ с размерностью, равной $\operatorname{dim} f(V)$, так что само множество $f(V)$ может быть полуаналитическим. Вторая оценка основана на теореме 3.2 . Если уровни имеют одинаковую размерность, то вьполнено

СлЕДСТвИЕ 3.5. Пусть $Z \subset W$ - локально аналитическое подмножество в $N$. Eсли $\operatorname{dim} f^{-1}(w)=k$ для всех $w \in Z, m o \operatorname{dim} f^{-1}(Z)=k+\operatorname{dim} Z$.

Для $z \in V$ обозначим через $l_{z} f$ росток уровня

$$
l_{z} f=\left(f^{-1}(f(z))\right)_{z}
$$

в точке $z$.

Tеорема 3.6.

(1) (полунепрерьвность) Отображсение $V \ni z \mapsto \operatorname{dim} l_{z} f$ полунепрерывно свер$x y:$ для всех $a \in V$ виполнено неравенство $\operatorname{dim} l_{z} f \leqslant \operatorname{dim} l_{a} f$ в некоторой окрестности точки а.

(2) Виполнено $\operatorname{dim} l_{z} f \geqslant \operatorname{dim}_{z} V-\operatorname{dim} f(V)$ для всех $z \in V$.

(3) (Картан-Реммерт) Для любого $k \in \mathbb{N}$ множество $\left\{z \in V: \operatorname{dim} l_{z} f \geqslant k\right\}$ аналитическое.

Множество $E$ назьвается аналитически получаемы.м, если сушествуют аналитические множества $V$ и $W$ такие, что $E=V \backslash W$.

Теорема 3.7. Пусть $V, W \subset M$ - аналитические множсества. Тогда замыкания любых связных компонент множества $V \backslash W$, любых открытых или замкнутых множеств в $V \backslash W$, и, в частности, множество $\overline{V \backslash W}$ представимы как обвединения простых компонент множества $V$, не содержащихся в $W$. Bсе вышеперечисленные множсетвв - аналитические. Семейство связных компонент множества $V \backslash W$ локально конечно.

Доказательства приведенных утверждений можно найти в [29] в II.1.4, II.3.4, V.1.1, II.3.6, V.3.2, V.3, IV.2.10. По [46] и [29; В.5.1] имеем: 
Tеорема 3.8 (теорема Реммерта о собственном отображении). Ecлu $f: X \rightarrow Y-$ собственное голоморфное отображсение между аналитическими пространствами, то его образ $f(X)$ аналитичен в $Y$. Образ любого аналитического подмножсества в $X$ аналитичен в $Y$.

\section{2. Основные результаты.}

ТЕОРема 3.9. Пусть Г удовлетворяет условиям (A1)-(А3). Предположим, что множество $\Omega^{\text {sing }}$ не пусто. Тогда справедлива следующая оценка.

(1) Мнохество существенных особенностей $\Omega^{\text {sing }}$ аналитично в $\Omega$ и для всех $\xi \in \Omega^{\text {sing }}$ имеем

$$
\max \{k-1, n-k+1\} \leqslant \operatorname{dim}_{\xi} \Omega^{\operatorname{sing}} \leqslant n-2 .
$$

В частности, $3 \leqslant k \leqslant n-1$ u $n \geqslant 4$.

(2) Пусть $\xi \in \Omega^{\text {sing }}$ - регулярная (гладкая) точка, $\xi=\lim _{i \rightarrow \infty} \xi_{i}, \xi_{i} \in \Omega^{(k)}, u$ $\mathbb{G}_{n-k}\left(\mathbb{C}^{n}\right) \ni \varkappa=\lim _{i \rightarrow \infty} \varkappa\left(\xi_{i}\right)$. Тогда $\varkappa \subset T_{\xi} \Omega^{\text {sing }}$.

В качестве следствия получаем, что в случае $k \leqslant 2$ все особенности устранимы.

ТЕОРема 3.10. Пусть Г удовлетворяет условиям (A1)-(A3) $c k \leqslant 2$. Тогда мно сество существенных особенностей $\Omega^{\operatorname{sing}}$ пусто и и допускает регулярное (голоморфное) продолжение в $\Omega$.

Оценки на размерность множества $\Omega^{\operatorname{sing}}$ в теоремах $3.9,3.10$ точны, т.е. для всех промежуточных размерностей существует отображение $\Gamma$, для которого промежуточная размерность достигается.

Tеорема 3.11. Для всеx $3 \leqslant k \leqslant n-1, p \geqslant k u 2 \leqslant d \leqslant \min \{k-1, n-k+1\} c y$ ществует голоморфное отображение $\Gamma: \mathbb{C}^{n} \rightarrow \mathbb{C}^{p}$, удовлетворяющее условиям (A1)-(A3) $c \operatorname{dim} \Omega^{\operatorname{sing}}=n-d$. Более того, можнно выбрать $\Gamma$ с $\Omega \backslash \Omega^{(k)}=\Omega^{\text {sing }}$.

Утверждения остаются справедливыми, если заменить $\mathbb{C}^{p}$ в определении $Г$ произвольным аналитическим пространством. Связь между свойствами (A1)-(A3) и слоениями с аффинными поверхностями уровня такая:

ТЕОрема 3.12. Пусть голоморфное отображсние $\Gamma: \Omega \rightarrow \mathbb{C}^{p}$ задано на открытом множсестве $\Omega \subset \mathbb{C}^{n}$. Пусть множество $\Omega_{0}$ открыто и плотно в $\Omega, u$ предположим, что для всех $\xi \in \Omega_{0}$ поверхность уровня $\Gamma^{-1}(\Gamma(\xi))$ через $\xi-а ф-$ финное подпространство в $\Omega$ коразмерности $k$ c $k<n$. Тогда $\Gamma$ удовлетворяет условиям (A1)-(A3) $и \Omega^{\operatorname{sing}} \subset \Omega \backslash \Omega_{0}$.

При условиях (Р1)-(Р3) имеем:

ТЕОРема 3.13. Пусть Г удовлетворяет условиям (P1)-(P3) с $k=1$, и пусть $(y, \xi) \in \Omega^{\operatorname{sing}}$. Тогда отображсене $\eta \mapsto \Gamma(y, \eta)$ постоянно.

Докажем сначала теорему 3.11. Так как $k-1 \leqslant n-d, d \leqslant n / 2$ и $d \leqslant k-1 \leqslant n-d$, можно рассмотреть отображение

$$
\Gamma:\left(x_{1}, \ldots, x_{d}, y_{1}, \ldots, y_{n-d}\right) \mapsto\left(\sum_{i=1}^{d} y_{i} x_{i}, y_{1}, \ldots, y_{k-1}, 0\right),
$$


где $0 \in \mathbb{C}^{n-k+2}$. Если не все $y_{i}$ с $1 \leqslant i \leqslant d$ равны нулю в точке, то $\operatorname{rank} D \Gamma$ в этой точке равен $k$. С другой стороны, очевидно, что точки с $y_{i}=0$ при всех $1 \leqslant i \leqslant d$ принадлежат $\Omega^{\operatorname{sing}}$.

Теперь докажем теорему 3.13 .

ЛЕмма 3.14. Пусть Г удовлетворяет условиям (P1)-(P2). Для $\omega \in \Omega^{\operatorname{sing}} u$ для любого $k$-мерного линейного подпространства $C$ в $\mathbb{C}^{n}$ существует последовательность $\omega_{j} \in \Omega^{(k)}$ такая, что $\omega_{j}$ сходится $\kappa \omega$ при $j \rightarrow \infty, \varkappa\left(\omega_{j}\right)$ сходится $\kappa \varkappa \in \mathbb{G}_{n-k}\left(\mathbb{C}^{n}\right)$ npu $j \rightarrow \infty u \varkappa \cap C \neq\{0\}$.

ДокаЗАтельство. Множество $G(C)=\left\{L \in \mathbb{G}_{n-k}\left(\mathbb{C}^{n}\right): L \cap C=\{0\}\right\}$ голоморфно диффеоморфно $\mathbb{C}^{k(n-k)}$ (см. [29; В.6.6] и [29; Рrop., p. 367]). Следовательно, если сушествует окрестность $U$ точки $\omega$ в $\Omega$ такая, что образ $\varkappa\left(U \cap \Omega^{(k)}\right)$ содержится в некотором компактном подмножестве в $G(C)$, то $\omega \notin \Omega^{\operatorname{sing}}$.

ЛЕмма 3.15. Пусть Г удовлетворяет условиям (P1)-(P3). Для каждой точ$\kappa u(y, \xi) \in \Omega^{\operatorname{sing}}$ и для кажсдого $k$-мерного линейного подпространства $C$ в $\mathbb{C}^{n}$ существует линейное подпространство $L$ в $C$ с $\operatorname{dim} L \geqslant 1$ такое, что для любого $l \in L$ виполнено

$$
\Gamma(y, \xi+l)=\Gamma(y, \xi)
$$

ДокАЗАТЕЛЬСТво. По лемме 3.14 существует последовательность $\omega_{j} \rightarrow(y, \xi)$ с $\omega_{j} \in \Omega^{(k)}$ такая, что сушествует предел $\lim _{j} \varkappa\left(\omega_{j}\right)=\varkappa$ и $\varkappa \cap C \neq\{0\}$. Возьмем $L=\varkappa \cap C$. По свойству (Р3) для любых $\omega_{j}=\left(y_{j}, \xi_{j}\right)$ и $z_{j} \in \varkappa\left(\omega_{j}\right)$ выполнено $\Gamma\left(y_{j}, \xi_{j}+z_{j}\right)=\Gamma\left(y_{j}, \xi_{j}\right)$. Утверждение следует из непрерьвности $\Gamma$.

Доказательство теоремы 3.13 следует из леммы 3.15 с $k=1$, так как в этом случае лемма 3.15 справедлива для любого $C$.

СлЕДСТВИЕ 3.16. Пусть Г удовлетворяет (A1)-(А3) с $k=1$. Тогда множество существенньх особенностей $\Omega^{\text {sing }}$ пусто.

ДокаЗАТЕльство. Пусть $\xi \in \Omega^{\text {sing }}$. По теореме 3.13 отображение $\eta \mapsto \Gamma(\eta)$ постоянно в $\Omega$ и $D_{\xi} \Gamma$ - нулевая матрища, противоречие с $k=1$.

Приведем пример слоения, зависяшего от двух параметров, для произвольного $1 \leqslant k \leqslant n-1$.

ПримЕР 3.17. Для $1 \leqslant k \leqslant n-1, m \geqslant 2$ зададим

$$
\Gamma(y, \xi)=\left(\begin{array}{c}
y_{1} \xi_{1}+y_{2} \xi_{2} \\
\vdots \\
y_{1} \xi_{1}+y_{2} \xi_{k+1}
\end{array}\right) \in \mathbb{C}^{k}
$$

где $y \in \mathbb{C}^{m}$ и $\xi \in \mathbb{C}^{n}$. Тогда матрица

$$
D_{\xi} \Gamma(y, \xi)=\left(\begin{array}{ccccccc}
y_{1} & y_{2} & 0 & \cdots & 0 & \cdots & 0 \\
y_{1} & 0 & y_{2} & \cdots & 0 & \cdots & 0 \\
\vdots & \vdots & \vdots & \vdots & \vdots & \vdots & \vdots \\
y_{1} & 0 & 0 & \cdots & y_{2} & \cdots & 0
\end{array}\right) \in \mathbb{C}^{k \times n}
$$


имеет максимальньй ранг, равньй $k$, и для всех $y$ с $y_{1} y_{2} \neq 0$ ядро $\varkappa(y, \xi)-$ линейная оболочка векторов

$$
\left(\begin{array}{c}
1 \\
-y_{1} / y_{2} \\
\vdots \\
-y_{1} / y_{2} \\
1 \\
0 \\
\vdots \\
0
\end{array}\right), \ldots,\left(\begin{array}{c}
1 \\
-y_{1} / y_{2} \\
\vdots \\
-y_{1} / y_{2} \\
0 \\
\vdots \\
0 \\
1
\end{array}\right)
$$

Следовательно, множество сушественных особенностей имеет вид

$$
\Omega^{\text {sing }}=\left\{(y, \xi) \in \mathbb{C}^{m} \times \mathbb{C}^{n}: y_{1}=y_{2}=0\right\} .
$$

Продолжим наш анализ множества сушественных особенностей $\Omega^{\text {sing }}$. В дальнейшем будем всюду предполагать, что отображение Г удовлетворяет условиям (A1), (A2) и (А3).

Сразу заметим, что условие (А 3$)$ аффинности уровней Г существенно для анализа. Условия (A1), (А2) сами по себе не влекут утверждение следствия 3.16. Например, если $\Gamma(\xi)=\left(\xi_{1}^{2}+\xi_{2}^{2}+\xi_{3}^{2}, 0,0\right) \in \mathbb{C}^{3}$, то несложно проверить, что $k=1$ и $\Omega^{\text {sing }}=\{0\}$. Однако, в этом случае $Г$ не постоянна на $\xi+\varkappa(\xi)$ и (А 3$)$ нарушается. Условие (А3) можно характеризовать следующим образом:

УТВЕРЖДЕНИЕ 3.18. Для любого $\xi \in \Omega^{(k)}$ отображсние $\Gamma$ постоянно на $(\xi+\varkappa(\xi)) \cap \Omega$, m.e.

$$
\Gamma(\zeta)=\Gamma(\xi)
$$

для всех $\zeta \in(\xi+\varkappa(\xi)) \cap \Omega$ тогда и только тогда, когда и постоянна на $(\xi+\varkappa(\xi)) \cap \Omega$, m.e.

$$
\varkappa(\xi) \subset \operatorname{ker} D \Gamma(\zeta)
$$

для всех $\zeta \in(\xi+\varkappa(\xi)) \cap \Omega$. При $\zeta \in(\xi+\varkappa(\xi)) \cap \Omega^{(k)}$ имеем равенство в (3.2).

ДоказАтЕльство. Дифференцирование (3.1) по базисным элементам в $\varkappa(\xi)$ влечет (3.2). Действительно, обозначим через $e_{j}(\xi), j=1, \ldots, n-k$, некоторый базис из векторов, порождающих линейное пространство $\varkappa(\xi)$, локально в $\Omega^{(k)}$. Тогда из $(3.1)$ вытекает, что

$$
\Gamma\left(\xi+t e_{j}(\xi)\right)=\Gamma(\xi), \quad j=1, \ldots, n-k,
$$

для небольших $t$ и $\zeta=\xi+t e_{j}(\xi)$. Дифференцируя $(3.3)$ в $t=0$, получаем

$$
D \Gamma\left(\xi+t e_{j}(\xi)\right) e_{j}(\xi)=0,
$$

что, в свою очередь, влечет (3.2). Для $\zeta \in \Omega^{(k)}$ размерности обеих сторон совпадают, поэтому имеем равенство. Обратно, из (3.2) следует, что $D \Gamma(\xi+\eta) \varkappa(\xi)=0$ для $\eta \in \varkappa(\xi)$ с $\xi+\eta \in \Omega$. Поэтому $\Gamma(\xi+\eta)$ постоянна на $\varkappa(\xi)$, т.е. справедливо равенство (3.1). Действительно, при $\zeta=\xi+t e_{j}(\xi)$ уравнение (3.4) можно переписать как $\partial \Gamma / \partial t\left(\xi+t e_{j}(\xi)\right)=0$, что влечет (3.1). Так как утверждение 3.18 верно локально в окрестности любой точки $\xi \in \Omega^{(k)}$ и отображение Г голоморфно, то оно вьполнено также глобально в $\Omega$. 
ЗАмЕчАниЕ 3.19. Отметим без доказательства, что происходит в случае, когда ранг падает на 1 . Пусть $\xi \in \Omega^{\text {sing }}$ и $\xi \in \Omega^{(k-1)}$, т.e. $\operatorname{rank} D \Gamma(\xi)=k-1$. Тогда $\Gamma(\xi+\lambda)=\Gamma(\xi)$ для всех $\lambda \in \operatorname{ker} D \Gamma(\xi)$ с $\xi+\lambda \in \Omega$.

График отображения $\varkappa$ имеет вид

$$
G=\left\{(\xi, L) \in \Omega \times \mathbb{G}_{n-k}\left(\mathbb{C}^{n}\right): \xi \in \Omega^{(k)}, L=\varkappa(\xi)\right\} .
$$

Пусть

$$
E=\left\{(\xi, L) \in \Omega \times \mathbb{G}_{n-k}\left(\mathbb{C}^{n}\right): L \subset \operatorname{ker} D \Gamma(\xi)\right\} .
$$

Множество $E$ - замкнутое аналитическое множество в $\Omega \times \mathbb{G}_{n-k}\left(\mathbb{C}^{n}\right)$ и

$$
G=\left(\Omega^{(k)} \times \mathbb{G}_{n-k}\left(\mathbb{C}^{n}\right)\right) \cap E=E \backslash\left\{(\xi, L) \in \Omega \times \mathbb{G}_{n-k}\left(\mathbb{C}^{n}\right): \xi \in \Omega \backslash \Omega^{(k)}\right\} .
$$

Таким образом, $G$ представимо как дополнение в $E$ к замкнутому аналитическому множеству $E \cap\left\{(\xi, L) \in \Omega \times \mathbb{G}_{n-k}\left(\mathbb{C}^{n}\right): \xi \in \Omega \backslash \Omega^{(k)}\right\}$ и, следовательно, аналитически получаемо.

ОПРЕДЕЛЕнИЕ 3.20 . Через $\bar{\varkappa}(\xi) \subset \mathbb{G}_{n-k}\left(\mathbb{C}^{n}\right)$ обозначим множество предельных точек последовательностей $\varkappa\left(\xi_{j}\right)$ при $\xi_{j} \rightarrow \xi, \xi_{j} \in \Omega^{(k)}$.

Для $V \subset \mathbb{G}_{n-k}\left(\mathbb{C}^{n}\right)$ через $\widetilde{V}$ обозначим множество

$$
\widetilde{V}=\bigcup_{L \in V} L
$$

Приведем основные свойства отображения $V \mapsto \widetilde{V}$.

УТВЕРЖДЕНИЕ 3.21. Если $V$ - аналитическое множество в $\mathbb{G}_{n-k}\left(\mathbb{C}^{n}\right)$, то $\widetilde{V}$ - аналитическое множество в $\mathbb{C}^{n}$. Более того,

$$
\operatorname{dim} \tilde{V} \leqslant \operatorname{dim} V+n-k .
$$

Eсли $\operatorname{dim} V \geqslant 1, m o \operatorname{dim} \widetilde{V} \geqslant n-k+1$.

ДокаЗАТЕЛЬСтво. Множество

$$
I=\left\{(\xi, L) \in \mathbb{C}^{n} \times \mathbb{G}_{n-k}\left(\mathbb{C}^{n}\right): \xi \in L\right\}
$$

- аналитическое множество в $\mathbb{C}^{n} \times \mathbb{G}_{n-k}\left(\mathbb{C}^{n}\right)$. Через $\pi_{1}:(\xi, L) \mapsto \xi$ обозначим проекцию из $\mathbb{C}^{n} \times \mathbb{G}_{n-k}\left(\mathbb{C}^{n}\right)$ в $\mathbb{C}^{n}$. Пусть $V$ - аналитическое множество в $\mathbb{G}_{n-k}\left(\mathbb{C}^{n}\right)$. Тогда $\widetilde{V}$ представимо в виде

$$
\widetilde{V}=\pi_{1}\left(I \cap\left(\mathbb{C}^{n} \times V\right)\right) .
$$

Множество $\mathbb{C}^{n} \times V$ и, следовательно, $I \cap\left(\mathbb{C}^{n} \times V\right)$ аналитичны в $\mathbb{C}^{n} \times \mathbb{G}_{n-k}\left(\mathbb{C}^{n}\right)$. По теореме Реммерта $3.5 \widetilde{V}$ аналитично, так как проекция $\pi_{1}$ собственная. Последнее следует из компактности грассманиана $\mathbb{G}_{n-k}\left(\mathbb{C}^{n}\right)$. 
Докажем теперь оценку на размерность. Через $\pi_{2}:(\xi, L) \mapsto L$ обозначим проекцию из $\mathbb{C}^{n} \times \mathbb{G}_{n-k}\left(\mathbb{C}^{n}\right)$ в $\mathbb{G}_{n-k}\left(\mathbb{C}^{n}\right)$. Имеем

$$
\pi_{2}\left(I \cap\left(\mathbb{C}^{n} \times V\right)\right)=V .
$$

Возьмем $v \in V \subset \mathbb{G}_{n-k}\left(\mathbb{C}^{n}\right)$. Уровень проекции $\pi_{2}$ в точке $v$ в $I \cap\left(\mathbb{C}^{n} \times V\right)$ состоит из точек $\{(\xi, v): \xi \in v\}$ и

$$
\operatorname{dim}\left(\pi_{2}^{-1}(v) \cap I \cap\left(\mathbb{C}^{n} \times V\right)\right)=\operatorname{dim} \widetilde{v}=n-k .
$$

Из следствия 3.5 вытекает $\operatorname{dim}\left(I \cap\left(\mathbb{C}^{n} \times V\right)\right)=n-k+\operatorname{dim} V$. Проекция $\pi_{1}$ не может увеличить размерность, из чего следует оценка в утверждении.

Предположим теперь, что $\operatorname{dim} V \geqslant 1$ и $\operatorname{dim} \widetilde{V}=n-k$. Множество $\widetilde{V}$ аналитично и поэтому имеет конечное число неприводимых компонент в каждой точке. По определению множества $\widetilde{V}$ каждая из этих компонент может быть только $(n-k)$-мерным подпространством в $\mathbb{C}^{n}$. Но это бы означало, что множество $V$ состоит из конечного числа изолированных точек, противоречие $\mathrm{c} \operatorname{dim} V \geqslant 1$. Конец доказательства.

Опишем основные свойства отображения $\varkappa$.

УТВЕРЖДЕНИЕ 3.22 .

(1) Множество $G$ в (3.5) аналитически получаемо, его замыкание $\bar{G}$ аналитично. Множество $\bar{\varkappa}(\xi)$ аналитично и связно для каждого $\xi \in \Omega$. Множество $\widetilde{\widetilde{\varkappa}}(\xi)$ аналитично в $\mathbb{C}^{n}$.

(2) Для любого $\xi \in \Omega$ ввпоолнено $\widetilde{\varkappa}(\xi) \subset \operatorname{ker} D \Gamma(\xi)$.

(3) Eсли $\xi \in \Omega^{\text {sing }}$, mо $\operatorname{dim} \bar{\varkappa}(\xi) \geqslant 1 u \operatorname{dim} \widetilde{\tilde{\varkappa}}(\xi) \geqslant n-k+1$. C другой стороньи, если $\xi \in \Omega \backslash \Omega^{\operatorname{sing}}$, то множество $\bar{\varkappa}(\xi)$ состоит из одного әлемента $L \in \mathbb{G}_{n-k}\left(\mathbb{C}^{n}\right) u \tilde{\tilde{\varkappa}}(\xi)=L$.

(4) Более того, если $\xi \in \Omega^{\text {sing }}$ и $C$ - неприводимая компонента множества $\widetilde{\bar{\varkappa}}(\xi)$, то $\operatorname{dim} C \geqslant n-k+1$. В частности, для всех $\xi \in \Omega^{\operatorname{sing}}$ имеем $\operatorname{dim}_{\xi}(\xi+\tilde{\widetilde{\varkappa}}(\xi)) \geqslant n-k+1$.

(5) $E$ сли $\xi \in \Omega^{(k-1)} \cap \Omega^{\text {sing }}, m o \tilde{\bar{\varkappa}}(\xi)=\operatorname{ker} D \Gamma(\xi) u \operatorname{dim} \tilde{\bar{\varkappa}}(\xi)=n-k+1$.

(6) Для любого $\xi \in \Omega$ отображение $\Gamma$ постоянно на $(\xi+\widetilde{\varkappa}(\xi)) \cap \Omega$.

ДокАЗАТЕльство. (1) Выше мы уже показали, что $G$ аналитически получаемо. Замыкание $\bar{G}$ аналитично как замыкание аналитически получаемого множества (теорема 3.7). Отсюда вытекает, что

$$
\bar{\varkappa}(\xi)=\left\{L \in \mathbb{G}_{n-k}\left(\mathbb{C}^{n}\right):(\xi, L) \in \bar{G}\right\}
$$

аналитично в $\mathbb{G}_{n-k}\left(\mathbb{C}^{n}\right)$. Действительно, $\{\xi\} \times \bar{\varkappa}(\xi)=\left(\{\xi\} \times \mathbb{G}_{n-k}\left(\mathbb{C}^{n}\right)\right) \cap \bar{G}-$ аналитическое множество и аналитичность $\bar{\varkappa}(\xi)$ следует из утверждения $3.3,(1)$.

Покажем, что $\bar{\varkappa}(\xi)$ связно. Пусть $U, V$ - открытые множества в $\mathbb{G}_{n-k}\left(\mathbb{C}^{n}\right)$, $U \cap V=\varnothing$ и $\bar{\varkappa}(\xi) \subset(U \cup V)$. Пусть $A=\left\{\eta \in \Omega^{(k)}: \varkappa(\eta) \in U\right\}$ и $B=\left\{\eta \in \Omega^{(k)}:\right.$ $\varkappa(\eta) \in V\}$. Тогда $A$ и $B$ - непересекаюшиеся открытые множества в $\Omega^{(k)}$. Сушествует открытая окрестность $W$ точки $\xi$ такая, что множество $W \cap \Omega^{(k)}$ связно и $W \cap \Omega^{(k)} \subset A \cup B$. Поэтому либо $A \cap W \cap \Omega^{(k)}=\varnothing$, либо $B \cap W \cap \Omega^{(k)}=\varnothing$. 
Получаем, что либо $\bar{\varkappa}(\xi) \cap U=\varnothing$, либо $\bar{\varkappa}(\xi) \cap V=\varnothing$. Таким образом, множество $\bar{\varkappa}(\xi)$ связно. Аналитичность $\widetilde{\varkappa}(\xi)$ в $\mathbb{C}^{n}$ следует из утверждения 3.21 .

(2) Утверждение следует из непрерывности $D$ Г и утверждения 3.18.

(3) Если $\xi \notin \Omega^{\operatorname{sing}}$, то $\bar{\varkappa}(\xi)$ состоит из одной точки и $\operatorname{dim} \varkappa(\xi)=0$. Обратно, при $\xi \in \Omega^{\operatorname{sing}}$ для любого $C \in \mathbb{G}_{k}\left(\mathbb{C}^{n}\right)$ множество

$$
\mathbb{G}_{n-k}\left(\mathbb{C}^{n}\right)_{C}=\left\{L \in \mathbb{G}_{n-k}\left(\mathbb{C}^{n}\right): L \cap C \neq\{0\}\right\}
$$

- гиперповерхность. Из условия $\bar{\varkappa}(\xi) \cap \mathbb{G}_{n-k}\left(\mathbb{C}^{n}\right)_{C} \neq \varnothing$ для всех $C \in \mathbb{G}_{k}\left(\mathbb{C}^{n}\right)$ (лемма 3.14$)$ следует, что множество $\bar{\varkappa}(\xi)$ бесконечно и $\operatorname{dim} \bar{\varkappa}(\xi)>0$. Из утверждения 3.21 получаем $\operatorname{dim} \frac{\widetilde{\varkappa}}{\varkappa}(\xi) \geqslant n-k+1$.

(4) Пусть $\operatorname{dim} C \leqslant n-k$. Тогда $C \in \mathbb{G}_{n-k}\left(\mathbb{C}^{n}\right), C \in \bar{\varkappa}(\xi)$. Множество $\bar{\varkappa}(\xi)$ связно по (1) и содержит больше одного элемента по (3). Следовательно, $C$ принадлежит замыканию регулярной части положительной размерности множества $\bar{\varkappa}(\xi)$. Отсюда следует, что $C$ содержится в неприводимой компоненте размерности $\geqslant n-k+1$ множества $\widetilde{\varkappa}(\xi)$, что противоречит предположению, что $C$ - неприводимая компонента множества $\widetilde{\varkappa}(\xi)$.

(5) Если $\xi \in \Omega^{(k-1)} \cap \Omega^{\text {sing }}$, то $\operatorname{ker} D \Gamma(\xi)$ - линейное подпространство в $\mathbb{C}^{n}$ коразмерности $k-1$. Из $\xi \in \Omega^{\operatorname{sing}}$ и $(2)$ следует, что $\widetilde{\bar{\varkappa}}(\xi)$ имеет коразмерность $k-1$. Докажем равенство в (5). Для $\eta \in \operatorname{ker} D \Gamma(\xi), \eta \neq 0$, через $L$ обозначим прямую через $\eta$. Тогда $L \subset \operatorname{ker} D \Gamma(\xi)$ и сушествует $C \in \mathbb{G}_{k}\left(\mathbb{C}^{n}\right)$ такое, что $L=\operatorname{ker} D \Gamma(\xi) \cap C$. По лемме 3.14 существует $\varkappa_{0} \in \bar{\varkappa}(\xi)$ с $\operatorname{dim}\left(\varkappa_{0} \cap C\right) \geqslant 1$. Тогда $\varkappa_{0} \cap C \subset \operatorname{ker} D \Gamma(\xi) \cap C=L$ и, следовательно, $\varkappa_{0} \cap C=L$. Отсюда получаем $\eta \in L=\varkappa_{0} \cap C \subset \varkappa_{0} \subset \frac{\widetilde{\varkappa}}{\varkappa}(\xi)$ и равенство между $\widetilde{\varkappa}(\xi)$ и $\operatorname{ker} D \Gamma(\xi)$.

(6) Для $\xi \in \Omega^{(k)}$ утверждение (6) равносильно (А3). Возьмем $\eta \in \widetilde{\widetilde{\varkappa}}(\xi)$ так, чтобы $\xi+\eta \in \Omega$. Тогда существуют последовательности $\xi_{j}, \eta_{j} \in \Omega^{(k)}$ такие, что $\eta_{j} \in \varkappa\left(\xi_{j}\right)$, $\xi_{j}+\eta_{j} \in \Omega, \xi_{j} \rightarrow \xi$ и $\eta_{j} \rightarrow \eta$. Условие (А3) означает, что $\Gamma\left(\xi_{j}+\eta_{j}\right)=\Gamma\left(\xi_{j}\right)$ и по непрерьвности $Г$ получаем $\Gamma(\xi+\eta)=\Gamma(\xi)$. Теорема доказана.

Отметим, что так как график отображения $\bar{\varkappa}$ аналитичен в $\Omega \times \mathbb{G}_{n-k}\left(\mathbb{C}^{n}\right)$, по теореме об аналитическомграфике (утверждение $3.3,(2))$ для голоморфности $\bar{\varkappa}$ достаточна его локальная ограниченность. При $k=n-1$ имеем $\mathbb{G}_{n-k}\left(\mathbb{C}^{n}\right)=\mathbb{P}\left(\mathbb{C}^{n}\right)$, и из теоремы Чоу $([6])$ следует, что множество $\bar{\varkappa}(\xi)$ алгебраическое и может быть задано как решение системы однородных полиномиальных уравнений в $\mathbb{C}^{n}$. Для произвольного $k$ можно использовать вложение Плюкера $\mathbb{G}_{n-k}\left(\mathbb{C}^{n}\right) \rightarrow \mathbb{P}\left(\Lambda^{n-k} \mathbb{C}^{n}\right)$ и теорему Чоу, чтобы показать, что $\bar{\varkappa}(\xi)$ алгебраично в $\Lambda^{n-k} \mathbb{C}^{n}$. Докажем теперь теорему 3.12 .

ДОКАЗАТЕЛЬСТВО ТЕОРЕМЫ 3.12. ПредполОЖим, что условия (А1) и (А2) выполнены для $\Gamma$ с некоторым $m \leqslant n$ вместо $k$. Тогда по теореме о неявной функции на множестве с максимальным рангом якобиана $D \Gamma$ уровни $\Gamma$ имеют размерность $n-m$. Поэтому $m=k$ и условия (A1) и (А2) выполнены с $m=k$. Для $\xi \in \Omega_{0}$ определим $\lambda(\xi)=\Gamma^{-1}(\Gamma(\xi))-\xi$. По предположению теоремы $\lambda$ задает отображение из $\Omega_{0}$ в $\mathbb{G}_{n-k}\left(\mathbb{C}^{n}\right)$. Отображение Г постоянно на $(\xi+\lambda(\xi)) \cap \Omega$, и поэтому, как и в утверждении 3.18 , имеем $\lambda(\xi) \subset \operatorname{ker} D \Gamma(\zeta)$ для $\zeta \in(\xi+\lambda(\xi)) \cap \Omega$. В частности, $\lambda(\xi)=\operatorname{ker} D \Gamma(\xi)$ для всех $\xi \in \Omega_{0} \cap \Omega^{(k)}$. Получаем условие $($ А 3$)$ на $\Omega_{0} \cap \Omega^{(k)}$. Множество $\Omega_{0} \cap \Omega^{(k)}$ плотно в $\Omega^{(k)}$ и в $\Omega$. Следовательно, для любого $\xi \in \Omega^{(k)}$ существует последовательность $\xi_{j} \in \Omega_{0} \cap \Omega^{(k)}$, сходящаяся к $\xi$. В силу компактности $\mathbb{G}_{n-k}\left(\mathbb{C}^{n}\right)$ получаем существование последовательности $\xi_{j}$ такой, что соответствующая последовательность 
$\varkappa\left(\xi_{j}\right)$ сходится к некоторому $\varkappa_{0} \in \mathbb{G}_{n-k}\left(\mathbb{C}^{n}\right)$. Без ограничения общности можно обозначить эту подпоследовательность также через $\xi_{j}$. Для любого $\eta \in\left(\xi+\varkappa_{0}\right) \cap \Omega$ сушествует последовательность $\eta_{j} \in\left(\xi_{j}+\varkappa\left(\xi_{j}\right)\right) \cap \Omega$ такая, что $\eta_{j}$ сходится к $\eta$. Из приведенного вьшше доказательства свойства (А 3$)$ для $\Omega_{0} \cap \Omega^{(k)}$ вытекает, что Г постоянна на $\xi_{j}+\varkappa\left(\xi_{j}\right)$ и $\Gamma\left(\eta_{j}\right)=\Gamma\left(\xi_{j}\right)$. Устремляя $j$ к бесконечности, имеем $\Gamma(\eta)=\Gamma(\xi)$ и $\eta \in \Gamma^{-1}(\Gamma(\xi))$. Так как рассуждение верно для всех $\eta \in\left(\xi+\varkappa_{0}\right) \cap \Omega$, получаем $\left(\xi+\varkappa_{0}\right) \cap \Omega \subset \Gamma^{-1}(\Gamma(\xi))$. По теореме о неявной функции уровень $\Gamma^{-1}(\Gamma(\xi))-$ гладкое аналитическое многообразие в $\Omega$ коразмерности $k$ и, следовательно, совпадает с $\xi+\varkappa_{0}$ локально в окрестности $\xi$. С другой стороны, $\varkappa$ голоморфна в $\xi$. Поэтому $\varkappa(\xi)=\varkappa_{0}$ и свойство (А3) вьполнено для всех $\xi \in \Omega^{(k)}$. Наконец, если $\xi \in \Omega^{\text {sing }}$, то по утверждению $3.22,(6)$, уровень $\Gamma$ через $\xi$ имеет коразмерность строго больше чем $k$ и, следовательно, $\xi \notin \Omega_{0}$. Конец доказательства.

Из утверждения 3.22 и компактности грассманиана следует, что отображение $\bar{\varkappa}: \Omega \rightarrow \mathbb{G}_{n-k}\left(\mathbb{C}^{n}\right)$ мероморфно (по определению 3.1 ). На $\Omega^{(k)}$ оно совпадает с голоморфным отображением $\varkappa$, его график аналитичен, и множества $\bar{\varkappa}(\xi)$ при $\xi \in \Omega \backslash \Omega^{(k)}$ компактны. Этот факт позволяет оценить размерность $\Omega^{\operatorname{sing}}$ сверху.

Tеорема 3.23. Множество $\Omega^{\operatorname{sing}}$ - аналитическое подмножество $\Omega$ c $\operatorname{dim} \Omega^{\text {sing }} \leqslant n-2$.

ДоказательСтво. Через $\pi$ обозначим сужение проекции $(\xi, L) \mapsto \xi$ на $\bar{G}$. По теореме Картана-Реммерта (теорема $3.6,(3))$ точки $g \in \bar{G}$, для которых размерность уровней $\pi^{-1}(\pi(g))$ в $g$ положительна, образуют аналитическое множество в $\bar{G}$, которое обозначим через $\Sigma$. Отсюда вытекает, что $\Sigma$ аналитично в $\Omega \times \mathbb{G}_{n-k}\left(\mathbb{C}^{n}\right)$. По компактностиграссманиана $\mathbb{G}_{n-k}\left(\mathbb{C}^{n}\right)$ проекция $\left.\pi\right|_{\bar{G}}$ задает собственное аналитическое отображение из $\bar{G}$ в $\Omega$. По теореме Реммерта о собственном отображении (теорема 3.8), множество $\pi(\bar{G})$ аналитическое в $\Omega$. Однако, $\pi(\bar{G} \backslash G)=\Omega^{\text {sing }}$, так что $\Omega^{\text {sing }}$ аналитическое подмножество в $\Omega$. Оценка на размерность следует из [46; Satz 26].

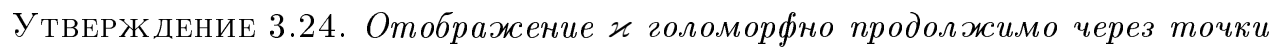
$\xi \in \Omega \backslash \Omega^{\text {sing. }}$. Множество $\Omega \backslash \Omega^{\operatorname{sing}}$ связно в $\Omega$, и поэтому $\varkappa$ допускает голоморфное продолжение в $\Omega \backslash \Omega^{\text {sing }}$. Это продолжение совпадает с сужением $\bar{\varkappa}$ на $\Omega \backslash \Omega^{\text {sing }}$. Более того, для любых $\xi \in \Omega \backslash \Omega^{\text {sing }} u \eta \in(\xi+\varkappa(\xi)) \cap\left(\Omega \backslash \Omega^{\text {sing }}\right)$ выполнено $\varkappa(\xi)=\varkappa(\eta)$.

ДокАЗАТЕльство. По определению множества $\Omega^{\operatorname{sing}}$ точки $\xi \in \Omega \backslash \Omega^{\operatorname{sing}}$ - устранимые особенности отображения $\varkappa$, которое голоморфно продолжимо в некоторую окрестность в $\Omega \backslash \Omega^{\text {sing }}$ каждой из этих точек. Множество $\Omega \backslash \Omega^{\text {sing }}$ открыто и связно по утверждению $3.3,(3)$. Таким образом, $\varkappa$ допускает глобальное голоморфное продолжение в $\Omega \backslash \Omega^{\operatorname{sing}}$, которое мы обозначим через $\varkappa_{0}$. Как множество график отображения $\varkappa_{0}$ лежит между графиками $\varkappa$ и $\bar{\varkappa}_{\mathrm{B}} \Omega \times \mathbb{G}_{n-k}\left(\mathbb{C}^{n}\right)$. Следовательно, замыкание графика $\varkappa_{0}$ совпадает с графиком $\bar{\varkappa}$, так что само $\varkappa_{0}$ совпадает с сужением $\bar{\varkappa}$ на $\Omega \backslash \Omega^{\text {sing. }}$. Предположим теперь, что $\xi$ и $\eta$ удовлетворяют условиям утверждения 3.24. Пусть $\xi_{j} \in \Omega^{(k)}, \xi_{j} \rightarrow \xi$. Тогда существует последовательность $\eta_{j} \in$ $\left(\xi_{j}+\varkappa\left(\xi_{j}\right)\right) \cap \Omega^{(k)}$ такая, что $\eta_{j} \rightarrow \eta$. Тогда $\varkappa\left(\eta_{j}\right) \rightarrow \varkappa(\eta)$, так как $\varkappa$ продолжима через $\eta$. Но $\varkappa\left(\xi_{j}\right)=\varkappa\left(\eta_{j}\right)$, так как $\xi_{j}, \eta_{j} \in \Omega^{(k)}$ и выполнено условие (А 3$)$. Получаем $\varkappa(\xi)=\lim _{j} \varkappa\left(\xi_{j}\right)=\varkappa(\eta)$. 
ЛЕмма 3.25. Для любого $\xi \in \Omega^{\text {sing }}$ имеем $\xi+\bar{\varkappa}(\xi) \subset \Omega \backslash \Omega^{(k)}$.

ДокАЗАТЕльСтво. Пусть $\xi \in \Omega^{\operatorname{sing}}$, и пусть $C$ - неприводимая компонента $\bar{\varkappa}(\xi)$ и $C^{0}$ - регулярная (гладкая) часть $C$. Так как $\Gamma=\Gamma(\xi)$ постоянна на $\xi+\widetilde{\widetilde{\varkappa}}(\xi)$, для любого $\eta^{0} \in C^{0} \mathrm{c} \xi+\eta^{0} \in \Omega$ вьполнено $T_{\eta} C^{0} \subset \operatorname{ker} D \Gamma\left(\xi+\eta^{0}\right)$. По утверждению 3.22, (4), $\operatorname{dim} \operatorname{ker} D \Gamma\left(\xi+\eta^{0}\right) \geqslant n-k+1$ и поэтому $\xi+\eta^{0} \in \Omega \backslash \Omega^{(k)}$. Так как $C^{0}$ плотно в $C$ и множество $\Omega$ открыто, к каждой точке $\eta \in C$ можно приблизиться точками $\eta^{0} \in C^{0}$ такими, что $\xi+\eta^{0} \in \Omega$. Поэтому $\xi+\eta \in \Omega \backslash \Omega^{(k)}$. Так как $\Omega \backslash \Omega^{(k)}$ замкнуто в $\Omega$, получаем $\xi+C \subset \Omega \backslash \Omega^{(k)}$. Так как это верно для всех неприводимых компонент в $\widetilde{\widetilde{\varkappa}}(\xi)$, имеем $\xi+\widetilde{\bar{\varkappa}}(\xi) \subset \Omega \backslash \Omega^{(k)}$.

Нам потребуются следуюшие две леммы. Нас интересуют раздутия с негладким центром. Если $S$ - гладкое подмногообразие коразмерности $k$ в $\mathbb{C}^{n}$, то слои раздутия над точками $S$ являются проективными пространствами $\mathbb{P}_{k-1}$ (см. [29; VII.5], [54; II.4, IV.3, VI.2.2]). Если $S$ аналитическое множество, то описанньй результат применим к внутренностям неприводимых компонент в $S$ разных размерностей, что приводит к возможно разным проективным пространствам в различных точках (см. [22; II.7, II.8]). Для нас будет важно, что происходит в особых точках.

ЛЕмма 3.26. Пусть отображение $f: \Omega \rightarrow \mathbb{C}^{p}$ голоморфно на открытом, связном множестве $\Omega$ в $\mathbb{C}^{n}$. Пусть $p \geqslant 2, f \not \equiv 0, S=\{\xi \in \Omega: f(\xi)=0\}$, $\xi_{0} \in S, \operatorname{dim}_{\xi_{0}} S \leqslant n-2$. Тогда отображсение

$$
F: \xi \mapsto \mathbb{C} f(\xi): \Omega \backslash S \rightarrow \mathbb{P}\left(\mathbb{C}^{p}\right)
$$

не допускает голоморфного продолжения через $\xi$.

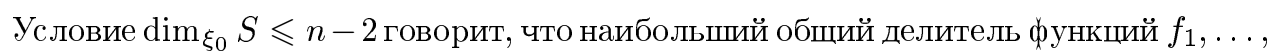
$f_{p}$ не обрашается в нуль в $\xi_{0}$.

ДокаЗАтельство. Рассуждая, как в лемме 3.14 , получаем, что если существует гиперплоскость $H$ в $\mathbb{P}\left(\mathbb{C}^{p}\right)$ такая, что $\bar{F}\left(\xi_{0}\right) \cap H$ пусто, то $F$ допускает голоморфное продолжение в окрестность $\xi_{0}$, которое также обозначим через $F$. Обратно, если $F$ голоморфна, то сушествует $H$, как выше. В этом случае сушествует индекс $i$ такой, что $\eta \in F\left(\xi_{0}\right), \eta \neq 0$ влечет $\eta_{i} \neq 0$. По непрерывности $F$ в $\xi_{0}$ сушествует $\varepsilon>0$ и такая окрестность $U$ точки $\xi_{0}$ в $\Omega$, что из $\xi \in U, \eta \in F(\xi)$ вытекает $\left|\eta_{i}\right| \geqslant \varepsilon\left|\eta_{j}\right|$ для всех $j \neq i$. Следовательно, если $\xi \in U \backslash S$, то $f_{i}(\xi) \neq 0$ или

$$
S \cap U \subset f_{i}^{-1}(0) \cap U \subset S,
$$

что противоречит $\operatorname{dim} f_{i}^{-1}(0)=n-1$, и $\operatorname{dim}_{\xi_{0}} S \leqslant n-2$. Конец доказательства.

Приведем без доказательства простую лемму линейной алгебры.

Лемма 3.27. Пусть $A \in \mathbb{C}^{p \times n} c \operatorname{rank} A=k$. Пусть максимальный ранг матриць $A$ достигается на подматрице $A_{\mathscr{L}}^{\mathscr{M}} \in \mathbb{C}^{k \times k}$ со строками и столбцами номерами в $\mathscr{L}$ и $\mathscr{M}$ соответственно, $\mathscr{L} \subset\{1, \ldots, p\}, \mathscr{M} \subset\{1, \ldots, n\}$. Тогда для любого $r, 1 \leqslant r \leqslant k, u\left\{\lambda_{i}\right\}_{i=1}^{r} \subset \mathscr{L}$ существуют $\left\{\mu_{i}\right\}_{i=1}^{r} \subset \mathscr{M}$ такие, что 
$\operatorname{det} A_{\lambda_{i}}^{\mu_{i}} \neq 0$, где $A_{\lambda_{i}}^{\mu_{i}} \in \mathbb{C}^{r \times r}-$ подматрица $A$, получаемая как пересечение строк $\lambda_{i}$ со столбиами $\mu_{i}, i=1, \ldots, r$.

Для упрощения обозначений будем писать $A_{i j}(\xi)$ вместо $\partial_{j} \Gamma_{i}(\xi)$. Отметим, что анализ справедлив и в более общем случае, когда $\varkappa(\xi)$ является слоением ядрами голоморфной матричнозначной функции $A: \Omega \rightarrow \mathbb{C}^{p \times n}$. В этом случае $\mathbb{G}_{n-k}\left(\mathbb{C}^{n}\right) \ni$ $\varkappa(\xi)=\operatorname{ker} A(\xi)$ для $\xi \in \Omega^{(k)}, \operatorname{rank} A(\xi) \leqslant k$ и $\Omega^{(k)} \neq \varnothing$.

УТВЕРЖДЕНИЕ 3.28. Если $\Omega^{\text {sing }}$ не nycmo, mo $\operatorname{dim}_{\xi} \Omega^{\operatorname{sing}} \geqslant k-1 \partial \Omega$ всех $\xi \in$ $\Omega^{\text {sing. }}$.

ДокаЗАТЕльство. По лемме 3.27 сушествуют подмножества $\mathscr{L}, \mathscr{M}$ в $\{1, \ldots, n\}$

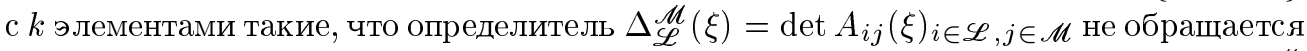
тождественно в нуль на $\Omega$. Через $Z_{\mathscr{L}, \mathscr{M}}$ обозначим множество нулей функции $\Delta_{\mathscr{L}}^{\mathscr{M}}$ в $\Omega$. Тогда для $\xi \in \Omega \backslash Z_{\mathscr{L}, \mathscr{M}}$ справедливо $\eta \in \operatorname{ker} A(\xi)$ тогда и только тогда, когда

$$
\sum_{j \in \mathscr{M}} A_{i j}(\xi) \eta_{j}+\sum_{m \notin \mathscr{M}} A_{i m}(\xi) \eta_{m}=0, \quad i \in \mathscr{L}
$$

Более того, если разрешить уравнения (3.6) через $\eta_{j}, j \in \mathscr{M}$, равенство (3.6) становится эквивалентным равенству

$$
\eta_{j}=\sum_{m \notin \mathscr{M}} \frac{f_{\mathscr{L}, \mathscr{M}}^{j m}(\xi)}{\Delta_{\mathscr{L}}^{\mathscr{M}}(\xi)} \eta_{m}, \quad j \in \mathscr{M}
$$

где $f_{\mathscr{L}, \mathscr{M}}^{j m}(\xi)$ полиномиальны как функции коэффициентов матрицы $A(\xi)$. Для $j \in \mathscr{M}$ через $Z_{\mathscr{L}, \mathscr{M}}^{j}$ обозначим обший нуль функций $f_{\mathscr{L}, \mathscr{M}}^{j m}, m \notin \mathscr{M}$, и $\Delta_{\mathscr{L}}^{\mathscr{M}}$ после деления их на наибольший общий множитель. Так как $\Omega \backslash Z_{\mathscr{L}, \mathscr{M}}$ плотно в $\Omega$ и правая часть в $(3.7)$ неоднозначна при $\xi \in \Omega^{\text {sing }}$, по лемме 3.26 имеем $Z_{\mathscr{L}, \mathscr{M}}^{j} \subset \Omega^{\text {sing }}$. Следовательно, $\operatorname{dim}_{\xi} Z_{\mathscr{L}, \mathscr{M}}^{j} \leqslant n-2$. Количество функций в определении $Z_{\mathscr{L}, \mathscr{M}}^{j}$ складьвается из $n-|\mathscr{M}|=n-k$ индексов $m \notin \mathscr{M}$ и дополнительной функции $\Delta_{\mathscr{L}}^{\mathscr{M}}$. Поэтому

$$
\operatorname{dim}_{\xi} Z_{\mathscr{L}, \mathscr{M}}^{j} \geqslant n-(n-k+1)=k-1
$$

и $\operatorname{dim}_{\xi} \Omega^{\operatorname{sing}} \geqslant k-1$.

ЗАмЕчАниЕ 3.29. Из доказательства утверждения 3.28 следует, что утверждение справедливо и в более общем случае, если заменить якобиан $D \Gamma(\xi)$ произвольной голоморфной матричнозначной функцией $A(\xi) \in \mathbb{C}^{p \times n}$ в $\Omega$. В этом случае аналогично определяем $k=\max _{\xi \in \Omega} \operatorname{rank} A(\xi)$ и $\varkappa(\xi)=\operatorname{ker} A(\xi) \in \mathbb{G}_{n-k}\left(\mathbb{C}^{n}\right)$ в $\Omega^{(k)}$.

УТВЕРЖДЕНИЕ 3.30. Пусть $k=n-1$. Если $\Omega^{\operatorname{sing}}$ не nусто, mo $\operatorname{dim} \Omega^{\operatorname{sing}}=$ $n-2$. Более того, $\operatorname{dim} A=n-2$ для любой неприводимой компоненты $A$ множсества $\Omega^{\text {sing }}$. 
ДокАЗАТЕЛЬСтво. Первое утверждение следует из утверждения 3.28 и теоремы 3.23. Возьмем $\mathscr{L}, \mathscr{M}$ как в доказательстве утверждения 3.28. Существуют индексы $l$ и $m, 1 \leqslant l, m \leqslant n, l \notin \mathscr{L}, m \notin \mathscr{M}$. Обозначим $\Delta_{l m}=\Delta_{\mathscr{L}}^{\mathscr{M}}, Z_{\mathscr{L}, \mathscr{M}}^{k}=Z_{l m}^{k}$ и $f_{\mathscr{L}, \mathscr{M}}^{j m}=f_{l m}^{k}$. Достаточно показать, что

$$
\Omega^{\operatorname{sing}}=\bigcup_{l, m, k: \Delta_{l m} \neq 0, f_{l m}^{k} \neq 0} Z_{l m}^{k}
$$

Из доказательства утверждения 3.28 видно, что $Z_{l m}^{k} \subset \Omega^{\text {sing }}$. Таким образом, достаточно доказать, что для любого $\xi \in \Omega^{\text {sing }}$ сушествуют индексы $l, m, k$ такие, что $\Delta_{l m} \not \equiv 0, f_{l m}^{k} \not \equiv 0$ и $\xi \in Z_{l m}^{k}$. Применяя лемму 3.27 с $r=n-1$, получаем, что для

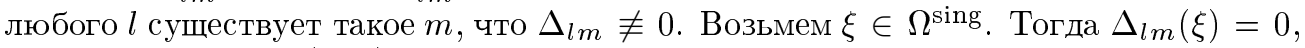
так как иначе $\xi \in \Omega^{(n-1)}$. Система (3.7) принимает вид

$$
\eta_{k}=\frac{f_{l m}^{k}(\xi)}{\Delta_{l m}(\xi)} \eta_{m}, \quad k \neq m
$$

Если для всех $k \in \mathscr{M}$ выполнено $f_{l m}^{k}(\xi) \equiv 0$, то все $\eta_{k} \equiv 0, k \neq m$, что означало бы, что слоение постоянно. Таким образом, для любого $m$ сушествуют $l$ и $k$ такие, что $f_{l m}^{k} \not \equiv 0$. Условие $\xi \notin Z_{l m}^{k}$ означает $f_{l m}^{k}(\xi) \neq 0$ или $\eta_{m}=0$. Если бы это было верно для всех $m$, то все $\eta_{m}=0$, что невозможно, так как $\Omega^{\text {sing }} \neq \varnothing$. Конец доказательства.

Теорема 3.31. Пусть $\operatorname{dim} \Omega^{\text {sing }}=k-1$, и пусть $A-$ регулярная (гладкая) компонента $\Omega^{\text {sing }}$ размерности $k-1$. Предположим, что существует $k$-мерная поверхность $S$, содержащая А и трансверсальная $\kappa \Omega \backslash \Omega^{(k)}$. Пусть $\eta \in \Omega \backslash \Omega^{\text {sing }}$. Тогда пересечение $\eta+\varkappa(\eta)$ и $S$ не мохет быть трансверсальным в точках множества $A$. В частности, при $k=n-1$ справедливо $\varkappa(\eta) \subset T_{\xi} A$ для всех $\eta \in \bar{\varkappa}\left(\Omega^{\text {sing }}\right) \backslash \Omega^{\text {sing }} u \xi=(\eta+\varkappa(\eta)) \cap A$.

ДокАЗАТЕльСТво. Предположим, что существует $\eta \in \Omega \backslash \Omega^{\operatorname{sing}}$ такая, что $\eta+\varkappa(\eta)$ и $S$ пересекаются трансверсально. Обозначим через $\delta: \Omega \rightarrow \mathbb{C}^{n-k}$ отображение, локально задающее $\varkappa(\xi)$ при $\xi \in \Omega^{(k)}$. Отображение $\delta$ мероморфно и неоднозначно в точках $\Omega^{\text {sing }}$. Пределы слоев $\varkappa(\xi)$ при $\xi \rightarrow A$ содержатся в дополнении к $\Omega^{(k)}$ в $\Omega$ (лемма 3.25 ) и поэтому трансверсальны поверхности $S$. Таким образом, все регулярные слои $\varkappa(\xi)$ с $\xi \in \Omega^{(k)}$ пересекают $S$ трансверсально, так как вьполнено

$$
\operatorname{dim} S+\left.\operatorname{dim} \tilde{\varkappa}\right|_{\Omega} ^{(k)}(\xi)=k+(n-k)=n .
$$

Следовательно, сужение $\left.\delta\right|_{S}$ неоднозначно во всех точках компоненты $A$. Но это противоречит тому, что точки, где отображение $\left.\delta\right|_{S}$ неоднозначно, удовлетворяют по крайней мере двум независимым аналитическим уравнениям и поэтому образуют множество по крайней мере коразмерности 2 в $S$. Противоречие доказывает первую часть теоремы. При $k=n-1$ имеем $\operatorname{dim} A=n-2$ по утверждению 3.30 . Если прямая $\eta+\varkappa(\eta)$ не касательная к $A$, то сушествует поверхность $S$, удовлетворяющая условиям первой части теоремы, что невозможно. Теорема доказана.

Докажем обобшение второй части теоремы 3.31 на остальные значения ранга $k$. 
УТВЕРЖДЕНИЕ 3.32. Пусть $A$-регулярная (гладкая) компонента $\Omega^{\text {sing }}$. Тогда для любого $\xi \in A$ справедливо $\widetilde{\widetilde{\varkappa}}(\xi) \subset T_{\xi} A$.

ДокАЗАтЕльСтво. Если для любого $\xi \in \Omega^{\operatorname{sing}}$ вьполнено $\xi+\widetilde{\bar{\varkappa}}(\xi) \subset \Omega^{\text {sing }}$, то утверждение очевидно. Пусть сушествует $\xi \in A$ с $\xi+\widetilde{\varkappa}(\xi) \not \subset \Omega^{\operatorname{sing}}$ и $\widetilde{\varkappa}(\xi) \not \subset T_{\xi} A$. Тогда в силу связности $\bar{\varkappa}(\xi)$ сушествуют различные $\varkappa_{1}, \varkappa_{2} \in \bar{\varkappa}(\xi)$, не содержашиеся в $\Omega^{\text {sing }}$ и в $T_{\xi} \Omega^{\text {sing }}$ (даже локально). Множество $\mathscr{K}_{t r}$ всех $H \in \mathbb{G}_{k+1}\left(\mathbb{C}^{n}\right)$ таких, что $H \cap \varkappa_{1}, H \cap \varkappa_{2}$ не содержатся в $T_{\xi} \Omega^{\operatorname{sing}}$, открыто и плотно в $\mathbb{G}_{k+1}\left(\mathbb{C}^{n}\right)$. Множество

$$
\mathscr{K}_{0}=\left\{H \in \mathbb{G}_{k+1}\left(\mathbb{C}^{n}\right): H \cap \varkappa_{1} \neq H \cap \varkappa_{2}\right\}
$$

открыто и плотно в $\mathbb{G}_{k+1}\left(\mathbb{C}^{n}\right)$. При $i=1,2$ множества

$$
\mathscr{K}_{i}=\left\{H \in \mathbb{G}_{k+1}\left(\mathbb{C}^{n}\right): \operatorname{dim} H \cap \varkappa_{i}=1\right\}
$$

открыты и плотны в $\mathbb{G}_{k+1}\left(\mathbb{C}^{n}\right)$. Таким образом, пересечение всех этих множеств также открыто и плотно в $\mathbb{G}_{k+1}\left(\mathbb{C}^{n}\right)$. Возьмем некоторое $H \in \mathscr{K}_{1} \cap \mathscr{K}_{2} \cap \mathscr{K}_{0} \cap \mathscr{K}_{t r}$. Пусть $\eta \in \Omega^{(k)}$ близка к $\xi$ с $\varkappa(\eta)$, близким к одной из $\varkappa_{i}$. По трансверсальности $\operatorname{dim}(\eta+\varkappa(\eta)) \cap(\xi+H)=1$. Множество $\Omega^{(k)} \cap(\eta+\varkappa(\eta))$ не пусто и поэтому открыто и плотно в $\Omega \cap(\eta+\varkappa(\eta))$. Следовательно, существует $\zeta \in \Omega^{(k)} \cap(\eta+\varkappa(\eta)), \zeta$ достаточно близко к $(\eta+\varkappa(\eta)) \cap(\xi+H)$, так что существует $H_{0} \in \mathscr{K}_{1} \cap \mathscr{K}_{2} \cap \mathscr{K}_{0} \cap \mathscr{K}_{t r}$ с $\zeta \in H_{0}$. Без ограничения обшности будем считать, что $H=H_{0}$. По (А3) имеем $\varkappa(\eta)=\varkappa(\zeta)$ и для отображения

$$
\gamma=\left.\Gamma\right|_{(\xi+H) \cap \Omega}
$$

справедливо ker $D \gamma(\zeta)=\varkappa(\zeta) \cap H$. Это пересечение одномерно и поэтому rank $D \gamma(\zeta)$ $=\operatorname{dim} H-1=k$. Более того, если $\theta \in \operatorname{ker} D \gamma(\zeta)$, то $\gamma(\theta)=\Gamma(\theta)=\Gamma(\zeta)=\gamma(\zeta)$, так как $\theta \in \varkappa(\zeta)$. Так, условия (A1), (А2) и (А3) выполнены для отображения $\gamma$. Возьмем $\eta_{j} \in \Omega^{(k)}$ так, что $\eta_{j} \rightarrow \xi$ и $\varkappa\left(\eta_{j}\right)$ сходится к одной из $\varkappa_{i}$. Так как множество

$$
\Omega^{(k)}(H)=\{\zeta \in(\xi+H) \cap \Omega: \operatorname{rank} D \gamma(\zeta)=k\}
$$

открыто и плотно в $(\xi+H) \cap \Omega$, сушествует последовательность $\zeta_{j} \in \Omega^{(k)}(H)$, сколь угодно близкая к $(\xi+H) \cap\left(\eta_{j}+\varkappa\left(\eta_{j}\right)\right)$. Тогда последовательность $\varkappa\left(\zeta_{j}\right)$ сколь угодно близка к $\varkappa\left(\eta_{j}\right)$. Имеем $\Omega^{(k)}(H) \subset \Omega^{(k)}$ и $\varkappa$ постоянна на открытом плотном подмножестве в $(\eta+\varkappa(\eta)) \cap \Omega, \eta \in \Omega^{(k)}$. Таким образом, в точке $\xi$ в $(\xi+H) \cap(\xi+\varkappa)$ получаем все элементы $H \cap \bar{\varkappa}(\xi)$ как пределы последовательностей $H \cap \varkappa\left(\zeta_{j}\right), \zeta_{j} \in \Omega^{(k)}(H)$, $\zeta_{j} \rightarrow \xi$. В частности, так как $H \in \mathscr{K}_{0}$, получаем в пределе две различные прямые $\varkappa_{i} \cap H \in \bar{\varkappa}(\xi) \cap H$. Это значит, что $\xi$ принадлежит множеству сушественных особенностей слоения $\zeta \mapsto \varkappa(\zeta) \cap H$ в $H$, соответствуюшего отображению $\gamma$. Кроме того,

$$
T_{\xi} \Omega^{\operatorname{sing}}(\gamma) \subset T_{\xi} \Omega^{\operatorname{sing}}(\Gamma) \cap H
$$

и из условия $H \in \mathscr{K}_{t r}$ получаем $H \cap \varkappa_{i} \not \subset T_{\xi} \Omega^{\operatorname{sing}}(\gamma)$. Противоречие с теоремой 3.31 и утверждением 3.30 , если применить их к отображению $\gamma$ с $n=k+1$. Теперь мы можем доказать теорему 3.9 . 
ДОКАЗАТЕЛЬСТВО ТЕОРЕМЫ 3.9. Вторая часть следует из утверждения 3.32. Из включения $\widetilde{\widetilde{\varkappa}}(\xi) \subset T_{\xi} \Omega^{\text {sing }}$ получаем оценку на размерность $\operatorname{dim}_{\xi} \Omega^{\text {sing }} \geqslant \operatorname{dim} \widetilde{\widetilde{\varkappa}}(\xi)$, что $\geqslant n-k+1$ по утверждению 3.22 . Вторая оценка следует из утверждения 3.28 .

3.3. Дальнейшие результаты. Обсудим кратко дальнейшие результаты, связанные со слоениями с аффинными слоями. Важным элементом в изучении множества $\Omega^{\text {sing }}$ оказьвается отношение эквивалентности, задаваемое слоением $\varkappa$. На множестве $\Omega^{(k)}$ две точки эквивалентны, если они принадлежат одному слою. Замькание такого отношения эквивалентности задает отношение на $\Omega$, для которого класс эквивалентности точки $\xi \in \Omega$ состоит из предельного конуса $\widetilde{\varkappa}(\xi)$. В частности, для $\xi \in \Omega^{\prime}=\Omega \backslash \Omega^{\operatorname{sing}}$ классы эквивалентности имеют такую же размерность, как и классы точек $\xi \in \Omega^{(k)}$.

УТВЕРЖДЕНИЕ 3.33. Множество

$$
\mathscr{R}=\left\{(\xi, \eta) \in \Omega^{\prime} \times \Omega^{\prime}: \eta-\xi \in \varkappa(\xi)\right\}
$$

- замкнутое гладкое аналитическое подмножество в $\Omega^{\prime} \times \Omega^{\prime}$ размерности $2 n-k$. Оно задает отношение эквивалентности на $\Omega^{\prime}$. Класс эквивалентности точки $\xi \in \Omega^{\prime}$ имеет вид

$$
\mathscr{R}(\xi)=\left\{\eta \in \Omega^{\prime}:(\xi, \eta) \in \mathscr{R}\right\} .
$$

Таким образом, множество $\Omega^{\prime}$ является обвединением классов әквивалентности $\mathscr{R}(\xi)$. Фактор-пространство $\Omega^{\prime} / \mathscr{R}$ - гладкое аналитическое многообразие размерности $k$.

Обозначим через $\rho$ естественную проекцию

$$
\rho: \Omega^{\prime} \ni \xi \mapsto \mathscr{R}(\xi) \in \Omega^{\prime} / \mathscr{R} .
$$

Факторизация $\Omega^{\prime} / \mathscr{R}$ имеет смысл, так как $\mathscr{R}$ замкнуто в $\Omega^{\prime} \times \Omega^{\prime}$. Фактор-пространство $\Omega^{\prime} / \mathscr{R}$ оснашается наиболее сильной хаусдорфовой топологией, для которой проекция $\rho$ непрерывна. Отображение Г постоянно на классе эквивалентности $\mathscr{R}(\xi)$. Поэтому сушествует аналитическое отображение $g: \Omega^{\prime} / \mathscr{R} \rightarrow \mathbb{C}^{p}$ такое, что

$$
\Gamma=g \circ \rho .
$$

Так как условие $\operatorname{rank} D \Gamma=k$ влечет $\operatorname{rank} D g \geqslant k$, касательное отображение к $g$ инъективно во всех точках и отображение $g$ задает погружение из $\Omega^{(k)} / \mathscr{R}$ в $\mathbb{C}^{p}$. Из факторизации (3.8) следует условие голоморфной факторизации для волновых фронтов (1.8).

Обозначим через $\pi_{1}$ и $\pi_{2}$ естественные голоморфные проекции

$$
\pi_{1}:(\xi, \eta) \mapsto \xi: \Omega \times \Omega \rightarrow \Omega, \quad \pi_{2}:(\xi, \eta) \mapsto \eta: \Omega \times \Omega \rightarrow \Omega .
$$

Множество $\mathscr{R} \cap\left(\Omega^{(k)} \times \Omega^{(k)}\right)$ представимо как дополнение к аналитическому множеству

$$
\left(\left(\Omega \backslash \Omega^{(k)}\right) \times \Omega\right) \cup\left(\Omega \times\left(\Omega \backslash \Omega^{(k)}\right)\right)
$$


в аналитическом множестве

$$
\{(\xi, \eta) \in \Omega \times \Omega: \eta-\xi \in \operatorname{ker} D \Gamma(\xi)\}
$$

в $\Omega \times \Omega$. Таким образом, $\mathscr{R}$ аналитически получаемо, и его замыкание $\overline{\mathscr{R}}$ в $\Omega \times \Omega-$ аналитическоеподмножество $\Omega \times \Omega$ (см. теорему 3.7$)$. Конус $\widetilde{\varkappa}(\xi)$ над $\bar{\varkappa}(\xi)$ представим в виде $\pi_{2}\left(\pi_{1}^{-1}(\{\xi\}) \cap \overline{\mathscr{R}}\right)$. По теореме 3.23 множество $\Omega^{\operatorname{sing}}$ аналитическое в $\Omega$. Поэтому множество $\overline{\mathscr{R}} \cap\left(\Omega^{\text {sing }} \times \Omega\right)$ также аналитическое в $\Omega \times \Omega$ и проекция $\pi_{1}: \overline{\mathscr{R}} \cap\left(\Omega^{\text {sing }} \times \Omega\right) \rightarrow$ $\Omega^{\text {sing }}$ сюръективна. Для второй проекции имеем

$$
\bigcup_{\xi \in \Omega^{\text {sing }}}(\xi+\widetilde{\widetilde{\varkappa}}(\xi))=\pi_{2}\left(\overline{\mathscr{R}} \cap\left(\Omega^{\operatorname{sing}} \times \Omega\right)\right) .
$$

УТВЕРЖДЕНИЕ 3.34. Множество $\mathscr{R}=\overline{\mathscr{R}} \cap\left(\Omega^{\prime} \times \Omega^{\prime}\right)$ аналитически получаемо, его замыкание $\overline{\mathscr{R}}$ - аналитическое подмножество в $\Omega \times \Omega$ и

$$
\operatorname{dim}(\overline{\mathscr{R}} \backslash \mathscr{R}) \leqslant 2 n-k-1
$$

Более того, вьполнень оченки:

$$
\begin{aligned}
& n-k+1 \leqslant \operatorname{dim} \pi_{2}\left(\overline{\mathscr{R}} \cap\left(\Omega^{\operatorname{sing}} \times \Omega\right)\right) \leqslant n-1, \\
& \operatorname{dim} \Omega^{\operatorname{sing}}+n-k+1 \leqslant \operatorname{dim}\left(\overline{\mathscr{R}} \cap\left(\Omega^{\operatorname{sing}} \times \Omega\right)\right) .
\end{aligned}
$$

ДокАЗАТЕльство. Первая оценка следует из того, что множество $\mathscr{R} \cap\left(\Omega^{(k)} \times \Omega^{(k)}\right)$ плотно в $\overline{\mathscr{R}}$ и, следовательно,

$$
\operatorname{dim}\left(\overline{\mathscr{R}} \backslash\left(\mathscr{R} \cap\left(\Omega^{(k)} \times \Omega^{(k)}\right)\right)\right)<\operatorname{dim} \overline{\mathscr{R}}=2 n-k
$$

Оценка (3.11) следует из (3.10), леммы 3.25 и $\operatorname{dim} \Omega \backslash \Omega^{(k)} \leqslant n-1$. Для $w \in \Omega^{\operatorname{sing}}$ уровень $\pi_{1}^{-1}(w) \cap\left(\overline{\mathscr{R}} \cap\left(\Omega^{\operatorname{sing}} \times \Omega\right)\right)$ имеет размерность $\geqslant n-k+1$ по утверждению 3.22 . По теореме 3.4 получаем (3.12).

Отметим, что оценка $\operatorname{dim} \Omega^{\operatorname{sing}} \leqslant n-2$ теоремы 3.23 следует из первой части утверждения 3.34 . Действительно, $\overline{\mathscr{R}} \cap\left(\Omega^{\operatorname{sing}} \times \Omega\right)$ содержится в $\overline{\mathscr{R}} \backslash \mathscr{R}$ и поэтому его размерность $\leqslant 2 n-k-1$. Уровни $\pi_{1}$ из $\overline{\mathscr{R}} \cap\left(\Omega^{\operatorname{sing}} \times \Omega\right)$ на $\Omega^{\operatorname{sing}}$ имеют размерность $\geqslant n-k+1$. Поэтому оценка $\operatorname{dim} \Omega^{\operatorname{sing}} \leqslant n-2$ следует из теоремы 3.4 .

В дальнейшем будем полагать, что множество $\Omega^{\text {sing }}$ не пусто. Приведем следующее утверждение без доказательства.

УТВЕРЖДЕНИЕ 3.35 .

(1) Выполнены включения $\Omega^{\text {sing }} \subset \pi_{2}\left(\overline{\mathscr{R}} \cap\left(\Omega^{\text {sing }} \times \Omega\right)\right) \subset \Omega \backslash \Omega^{(k)}$.

(2) Eсли $\Omega^{\operatorname{sing}}=\pi_{2}\left(\overline{\mathscr{R}} \cap\left(\Omega^{\text {sing }} \times \Omega\right)\right)$, то замыкание $\overline{\mathscr{R}}$ представимо как оббединение отношений әквивалентности на $\left(\Omega \backslash \Omega^{\text {sing }}\right) \times\left(\Omega \backslash \Omega^{\text {sing }}\right)$ и на $\Omega^{\operatorname{sing}} \times \Omega^{\operatorname{sing}}$. Иными словами, имеем

$$
\overline{\mathscr{R}} \cap\left(\Omega^{\text {sing }} \times \Omega\right) \subset \Omega^{\text {sing }} \times \Omega^{\text {sing }} .
$$


Более того, виполнено

$$
n-k+1 \leqslant \operatorname{dim}_{\xi} \Omega^{\operatorname{sing}}
$$

для всех $\xi \in \Omega^{\text {sing }}$.

(3) $E$ c $л u \Omega^{\text {sing }} \neq \pi_{2}\left(\overline{\mathscr{R}} \cap\left(\Omega^{\operatorname{sing}} \times \Omega\right)\right) u$

$$
l=\max _{\eta \in \pi_{2}\left(\overline{\mathscr{R}} \cap\left(\Omega^{\text {sing }} \times \Omega \backslash \Omega^{\text {sing }}\right)\right)} \operatorname{dim}\left(\Omega^{\text {sing }} \cap(\eta+\varkappa(\eta))\right),
$$

mo

$$
\operatorname{dim} \pi_{1}\left(\mathscr{R} \cap\left(\Omega^{\text {sing }} \times \Omega \backslash \Omega^{\text {sing }}\right)\right) \leqslant k-2+l .
$$

(4) Если в дополнение $\kappa(3)$ имеем $\operatorname{dim}\left(\overline{\mathscr{R}} \cap\left(\Omega^{\operatorname{sing}} \times \Omega \backslash \Omega^{\operatorname{sing}}\right)\right)=\operatorname{dim}(\overline{\mathscr{R}} \cap$ $\left.\left(\Omega^{\text {sing }} \times \Omega\right)\right)$ uлu $\operatorname{dim} \pi_{1}\left(\overline{\mathscr{R}} \cap\left(\Omega^{\text {sing }} \times \Omega \backslash \Omega^{\operatorname{sing}}\right)\right)=\operatorname{dim} \Omega^{\text {sing }}, m o$

$$
\operatorname{dim} \Omega^{\text {sing }}+2 \leqslant \operatorname{dim} \pi_{2}\left(\overline{\mathscr{R}} \cap\left(\Omega^{\text {sing }} \times \Omega\right)\right) .
$$

(5) Пусть $\Omega^{\operatorname{sing}} \neq \pi_{2}\left(\overline{\mathscr{R}} \cap\left(\Omega^{\operatorname{sing}} \times \Omega\right)\right)$. Тогда для всех $\xi \in \Omega^{\operatorname{sing}}$ c $\xi \notin \pi_{1}(\overline{\mathscr{R}} \cap$ $\left.\left(\Omega^{\text {sing }} \times \Omega \backslash \Omega^{\text {sing }}\right)\right)$ имеем $\xi+\widetilde{\varkappa}(\xi) \subset \Omega^{\text {sing }}$. Более того, в этом случае $k \geqslant 3 u \operatorname{dim}_{\xi} \Omega^{\operatorname{sing}} \geqslant n-k+1$.

Отсюда вытекают следующие оценки:

УТВЕРЖДЕНИЕ 3.36.

(1) Подмножество $\overline{\mathscr{R}} \cap\left(\Omega^{\operatorname{sing}} \times \Omega \backslash \Omega^{\text {sing }}\right) \subset \overline{\mathscr{R}} \cap\left(\Omega^{\text {sing }} \times \Omega\right)$ собственное.

(2) Eсли $\pi_{1}\left(\overline{\mathscr{R}} \cap\left(\Omega^{\text {sing }} \times \Omega \backslash \Omega^{\text {sing }}\right)\right) \neq \Omega^{\text {sing }}$, mo $\operatorname{dim}_{\xi} \Omega^{\text {sing }} \geqslant n-k+1$ для всех $\xi \in \Omega^{\text {sing }} \backslash \pi_{1}\left(\overline{\mathscr{R}} \cap\left(\Omega^{\text {sing }} \times \Omega \backslash \Omega^{\text {sing }}\right)\right) u k \geqslant 3$.

(3) $\operatorname{Ecлu~} \pi_{1}\left(\overline{\mathscr{R}} \cap\left(\Omega^{\text {sing }} \times \Omega \backslash \Omega^{\text {sing }}\right)\right)=\Omega^{\text {sing }}$, mo $\operatorname{dim} \Omega^{\text {sing }} \leqslant n-3$.

\section{4. Аффинные слоения градиентного типа}

4.1. Локализация. Продолжаем предполагать всюду, что голоморфное отображение Г удовлетворяет условиям (A1)-(А3) введения. Без ограничения общности можно предположить, что центр координат пространства $\mathbb{C}^{n}$ лежит в открытом множестве $\Omega$. Очевидно, если $\varkappa$ непрерывно в нуле, т.е. если $0 \notin \Omega^{\operatorname{sing}}$, то сушествует окрестность нуля $U$, не пересекающаяся с $\Omega^{\operatorname{sing}}$, такая, что все слои через точки в $U$ близки к слою $\varkappa(0)$. Поэтому существует $k$-мерное линейное подпространство в $\mathbb{C}^{n}$, трансверсальное всем $\varkappa(\xi)$ с $\xi \in U$. С другой стороны, предположим, что $0 \in \Omega^{\operatorname{sing}}$, и возьмем открытую окрестность нуля $U \subset \Omega$. Тогда для любого $H \in \mathbb{G}_{k}\left(\mathbb{C}^{n}\right)$ множесTBO

$$
U_{H}=\left\{\xi \in U \cap \Omega^{(k)}: \varkappa(\xi) \cap H=\{0\}\right\}
$$

либо пусто, либо открыто и плотно в $U \cap \Omega^{(k)}$ и, следовательно, в $U$. Таким образом, для $H$ с непустьм множеством $U_{H}$ можно выбрать такую систему координат в окрестности нуля, что $H$ параметризовано точками $(h, 0) \in \mathbb{C}^{k} \times \mathbb{C}^{n-k}$ и на открытом плотном подмножестве множества $\Omega^{(k)}$ все слои трансверсальны к $k$-мерному подпространству $(h, 0) \in \mathbb{C}^{k} \times \mathbb{C}^{n-k}$. Через $\pi_{h}: \mathbb{C}^{n} \rightarrow \mathbb{C}^{k}$ обозначим проекцию на первые $k$ 
компонент векторов из $\mathbb{C}^{n}$. Через $\Omega_{h}^{(k)}$ обозначим множество всех точек $h \in \pi_{h}(\Omega)$ с $(h, 0) \in \Omega^{(k)}$. Множество всех $h \in \pi_{h}(\Omega)$ с $(h, 0) \in \Omega$ обозначим через $\Omega_{h}$.

Для простоты в обозначениях отождествим пространства $\mathbb{C}^{n}$ и $\mathbb{C}^{1 \times n}$, т.е. будем рассматривать элементы $\mathbb{C}^{n}$ как строки из $n$ комплексных чисел. В этих обозначениях параметризуем слоение в помошью матричнозначной функции $R: \Omega_{h}^{(k)} \subset \mathbb{C}^{k} \rightarrow$ $\mathbb{C}^{(n-k) \times k}$. Слои $\varkappa(h, 0)$ параметризуем с помощью $\lambda$ в некоторой окрестности нуля в $\mathbb{C}^{n-k}:$ локально вьполнено

$$
\varkappa(h, 0)=\left\{(h+\lambda R(h), \lambda), \lambda \in \mathbb{C}^{n-k}\right\}=\left\{\left(h+\sum_{i=1}^{n-k} \lambda_{i} R_{i}(h), \lambda\right), \lambda \in \mathbb{C}^{n-k}\right\} .
$$

Строки матрицы $R(h)$ будем обозначать через $R_{i}(h) \in \mathbb{C}^{k}, i=1, \ldots, n-k$, и компоненты каждой строки $R_{i}(h)$ через $R_{i}^{j}(h), j=1, \ldots, k$. Колонку $j$ матришы $R(h)$ обозначим через $R^{j}(h) \in \mathbb{C}^{n-k}, j=1, \ldots, k$, и ее компоненты через $R_{i}^{j}(h)$. Таким образом, во введенных обозначениях имеем:

$$
\begin{aligned}
R & \left.=\left(\begin{array}{cccc}
\overbrace{R_{1}^{1}} & R_{1}^{2} & \ldots & R_{1}^{k} \\
\ldots \ldots & \ldots \ldots \ldots \ldots \\
R_{n-k}^{1} & R_{n-k}^{2} & \ldots & R_{n-k}^{k}
\end{array}\right)\right\}(n-k), \\
R_{l} & =\left(\begin{array}{cccc}
R_{l}^{1} & R_{l}^{2} & \ldots & R_{l}^{k}
\end{array}\right), \\
D R_{l} & =\left(\begin{array}{ccc}
\partial_{1} R_{l}^{1} & \ldots & \partial_{k} R_{l}^{1} \\
\ldots \ldots & \ldots & \ldots \\
\partial_{1} R_{l}^{k} & \ldots & \partial_{k} R_{l}^{k}
\end{array}\right) .
\end{aligned}
$$

Постоянство Г на $\varkappa$ в условии (А3) теперь записьвается как

$$
\Gamma(h+\lambda R(h), \lambda)=\Gamma(h, 0), \quad \forall h \in U \cap \Omega_{h}^{(k)}, \quad \lambda \in V,
$$

в некоторой окрестности нуля $U \times V \subset\left(\mathbb{C}^{k} \times \mathbb{C}^{n-k}\right) \cap \Omega$.

Лемма 4.1. Зададим $\gamma: \Omega_{h} \rightarrow \mathbb{C}^{p}$ с помощью $\gamma(h)=\Gamma(h, 0)$. Тогда

$$
\left\{\partial_{i} \Gamma(h+\lambda R(h), \lambda)\right\}_{i=1}^{k}\left[I_{k}+\sum_{l=1}^{n-k} \lambda_{l} D R_{l}(h)\right]=D \gamma(h)
$$

для всех $h \in \Omega_{h}^{(k)}$.

ДокАЗАТЕльСТво. Дифференшируя $\Gamma_{j}$ в $(4.2)$ по $h_{m}$, получаем

$$
\begin{aligned}
\partial_{m} \Gamma_{j}(h, 0)= & \frac{\partial}{\partial h_{m}} \Gamma_{j}(h+\lambda R(h), \lambda) \\
= & \partial_{m} \Gamma_{j}(h+\lambda R(h), \lambda)\left[1+\lambda \partial_{m} R^{m}(h)\right] \\
& +\sum_{1 \leqslant i \leqslant m, i \neq m} \partial_{i} \Gamma_{j}(h+\lambda R(h), \lambda)\left[\lambda \partial_{m} R^{i}(h)\right] .
\end{aligned}
$$

В матричных обозначениях это есть (4.3).

Группа $G L_{n}(\mathbb{C})$ действует на Г естественным образом. Для $A \in G L_{n}$ обозначим

$$
\Gamma_{A}(\xi)=\Gamma(A \xi) .
$$


УТВЕРЖДЕНИЕ 4.2. Пусть Г удовлетворяет условиям (A1)-(А3).

(1) Для всех $A \in G L_{n}$ отображсение $\Gamma_{A}: A^{-1}(\Omega) \rightarrow \mathbb{C}^{p}$ удовлетворяет (A1)-(А3). Кроме того, имеем $\Omega^{(k)}\left(\Gamma_{A}\right)=A^{-1}\left(\Omega^{(k)}(\Gamma)\right)$ и $\Omega^{\operatorname{sing}}\left(\Gamma_{A}\right)=$ $A^{-1}\left(\Omega^{\operatorname{sing}}(\Gamma)\right)$.

(2) Cуществует $A \in G L_{n}$ такая, что отображсние $\gamma_{A}:\left(A^{-1}(\Omega)\right)_{h} \rightarrow \mathbb{C}^{p}$, заданное с помощью $\gamma_{A}(h)=\Gamma_{A}(h, 0)$, удовлетворяет

$$
\operatorname{rank} D \gamma_{A}(h)=k
$$

для всех $\left(A^{-1}(\Omega)\right)_{h}$.

ДоказАтельство. Утверждение (1) очевидно. Докажем (2). Через $i_{1}, \ldots, i_{k}$ обозначим минимальные индексы, для которых существуют $j_{1}, \ldots, j_{k}$ и $\xi \in \Omega^{(k)}$ с

$$
\operatorname{det}\left(\begin{array}{ccc}
\partial_{i_{1}} \Gamma_{j_{1}}(\xi) & \ldots & \partial_{i_{k}} \Gamma_{j_{1}}(\xi) \\
\ldots \ldots \ldots & \ldots & \ldots \\
\partial_{i_{1}} \Gamma_{j_{k}}(\xi) & \ldots & \partial_{i_{k}} \Gamma_{j_{k}}(\xi)
\end{array}\right) \neq 0
$$

Для $\tau \neq 0$ определим $A_{\tau} \in G L_{n}$ через

$$
A_{\tau} \zeta=\tau \zeta+\sum_{l=1}^{k} \zeta_{l} e_{i_{l}}
$$

где $e_{l}-$ стандартный базис в $\mathbb{C}^{n}$. По первой части утверждения отображение $\Gamma_{A_{\tau}}$ удовлетворяет условиям (A1)-(А3). Якобиан отображения $\gamma_{A_{\tau}}$ имеет вид

$$
D \gamma_{A_{\tau}}(\zeta)=\left(\begin{array}{ccc}
\tau \partial_{1} \Gamma_{1}\left(A_{\tau} \zeta\right)+\partial_{i_{1}} \Gamma_{1}\left(A_{\tau} \zeta\right) & \ldots & \tau \partial_{k} \Gamma_{1}\left(A_{\tau} \zeta\right)+\partial_{i_{k}} \Gamma_{1}\left(A_{\tau} \zeta\right) \\
\ldots \ldots \ldots \ldots \ldots \ldots \ldots \ldots \ldots \ldots \ldots \ldots \ldots \ldots \ldots \ldots \ldots \ldots \ldots \ldots \ldots & \ldots \ldots \ldots \ldots
\end{array} \ldots\right.
$$

В частности, эта матрица содержит блок

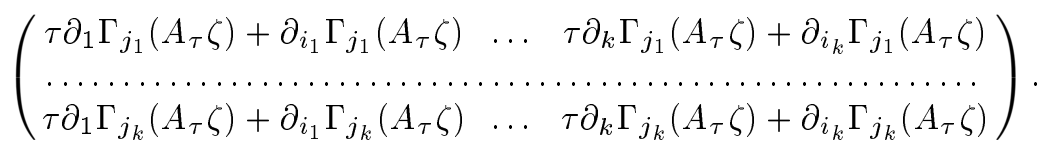

Определитель этого блока ненулевой для достаточно небольших $\tau$ и для некоторого $\zeta \in A_{\tau}^{-1}\left(\Omega^{(k)}\right)$ в силу выбора индексов $i_{1}, \ldots, i_{k}$ и $j_{1}, \ldots, j_{k}$ в (4.4). Ввиду аналитичности Г блок (4.6) невырожден на дополнении к некоторому собственному аналитическому подмножеству в $\Omega_{h}^{(k)}$, что завершает доказательство.

Ввиду утверждения 4.2 без ограничения обшности можно предположить, что отображение $\gamma$ в (4.3) удовлетворяет условию невырожденности утверждения 4.2, (2). Для глобальных слоений получаем

УТВЕРЖДЕНИЕ 4.3. Пусть $Г: \mathbb{C}^{n} \rightarrow \mathbb{C}^{p}-$ целая голоморфная функиия. Тогда для любого $h \in \Omega_{h}^{(k)}$ из условия $\operatorname{rank} D \gamma(h)=k$ вытекает, что матрици $D R_{l}(h)$ нильпотентны для всех $1 \leqslant l \leqslant n-k$. 
ДокАЗАТЕЛЬСТвО. Предположим, что есть не нильпотентная матрица $D R_{l_{0}}(h) \mathrm{c}$ ненулевым собственным значением $\mu \in \mathbb{C}$. Обе части уравнения (4.3) локально голоморфны по $\lambda$. Так как $\Gamma$ - целая функция, по теореме об аналитическом продолжении уравнение (4.3) вьполнено для всех $\lambda \in \mathbb{C}$. Подставляя $\lambda_{l}=-\mu^{-1} \delta_{l l_{0}}$, получаем вырожденность матрицы $D \gamma(h)$ и противоречие.

ПримеР 4.4. Г: $\mathbb{C}^{4} \rightarrow \mathbb{C}^{4}$ с помошњю

$$
\Gamma\left(\xi_{1}, \xi_{2}, \xi_{3}, \xi_{4}\right)=\left(\xi_{1} \xi_{2}+\xi_{3} \xi_{4}, \xi_{2}, \xi_{3}, 0\right) .
$$

Слоение, задаваемое Г, глобально, и для Г и его сужения

$$
\gamma\left(h_{1}, h_{2}, h_{3}\right)=\left(h_{1} h_{2}, h_{2}, h_{3}, 0\right)
$$

имеем

$$
D \Gamma(\xi)=\left(\begin{array}{cccc}
\xi_{2} & \xi_{1} & \xi_{4} & \xi_{3} \\
0 & 1 & 0 & 0 \\
0 & 0 & 1 & 0 \\
0 & 0 & 0 & 0
\end{array}\right), \quad D \gamma(h)=\left(\begin{array}{ccc}
h_{2} & h_{1} & 0 \\
0 & 1 & 0 \\
0 & 0 & 1 \\
0 & 0 & 0
\end{array}\right)
$$

Утверждение 4.3 вьполнено для всех $h \in \mathbb{C}^{3} \mathrm{c} h_{2} \neq 0$. Локализация $R$ слоения $\varkappa$ удовлетворяет уравнению $D \Gamma(h, 0)\left(\begin{array}{c}R(h) \\ 1\end{array}\right)=0$, из которого получаем $R^{1}(h)=-h_{3} / h_{2}$, $R^{2}(h)=0, R^{3}(h)=0$, с нильпотентной матрицей $D R(h)=\left(\begin{array}{ccc}0 & h_{3} h_{2}^{-2} & -h_{2}^{-1} \\ 0 & 0 & 0 \\ 0 & 0 & 0\end{array}\right)$.

ПримеР 4.5. Ранг матрицы $D \Gamma$ может падать больше чем на единицу в точках $\Omega \backslash \Omega^{(k)}$. Для $\Gamma_{1}(\xi)=\left(\xi_{1} \xi_{2}+\xi_{3} \xi_{4}, \xi_{2}^{2}, \xi_{3}^{2}, 0\right)$ и $\Gamma_{2}(\xi)=\frac{1}{2}\left(\left(\xi_{1} \xi_{2}+\xi_{3} \xi_{4}\right)^{2}, \xi_{2}^{2}, \xi_{3}^{2}, 0\right)$ имеем

$$
D \Gamma_{1}(\xi)=\left(\begin{array}{cccc}
\xi_{2} & \xi_{1} & \xi_{4} & \xi_{3} \\
0 & 2 \xi_{2} & 0 & 0 \\
0 & 0 & 2 \xi_{3} & 0 \\
0 & 0 & 0 & 0
\end{array}\right), D \Gamma_{2}(\xi)=\left(\begin{array}{cccc}
\xi_{2} \alpha(\xi) & \xi_{1} \alpha(\xi) & \xi_{4} \alpha(\xi) & \xi_{3} \alpha(\xi) \\
0 & \xi_{2} & 0 & 0 \\
0 & 0 & \xi_{3} & 0 \\
0 & 0 & 0 & 0
\end{array}\right)
$$

с $\alpha(\xi)=\xi_{1} \xi_{2}+\xi_{3} \xi_{4}$. Таким образом, $\left.\operatorname{rank} D \Gamma_{1}\right|_{\xi_{2}=\xi_{3}=0}=1,\left.\operatorname{rank} D \Gamma_{1}\right|_{\xi=0}=0$ и $\left.\operatorname{rank} D \Gamma_{2}\right|_{\xi_{2}=\xi_{3}=0}=0$.

4.2. Слоения градиентного типа. В дальнейшем мы докажем результаты одновременно для двух скалярных полей $\mathbb{R}$ и $\mathbb{C}$, которые обозначим через $\mathbb{K}$. В этих обозначениях при $m \geqslant 1$ пространство $C^{m}\left(\mathbb{K}^{n}\right)$ означает $C^{m}\left(\mathbb{R}^{n}\right)$ при $\mathbb{K}=\mathbb{R}$ и пространство голоморфных функций при $\mathbb{K}=\mathbb{C}$.

Рассмотрим слоение подпространствами коразмерности $k$, соответствуюшее отображению Г градиентного типа

$$
\Gamma(\xi)=\nabla \psi(\xi)
$$

с некоторой функцией $\psi: \Omega \rightarrow \mathbb{K}$. Если отображение $Г$ имеет вид (4.7), то соответствуюшее слоение будем называть слоением градиентного типа. Слоения такого типа возникнут в разделе 5 , где Г принимает вид (4.7) с производящей функцией $\phi$ 
или ее неоднородной версией $\psi$. В этом случае $D \Gamma=D^{2} \psi$ и слои $\varkappa$ отображения $\Gamma$ соответствуют уровням градиента $\nabla \psi$. В общем, будем предполагать определенную гладкость, однако отметим, что последуюшие утверждения верны на области, где все производные имеют смысл, т.е. по крайней мере на открытом множестве $\Omega^{(k)}$ c rank $D^{2} \psi=k$. Мы сохраняем обозначения (4.1) для отображения

$$
R: \Omega_{h}^{(k)} \subset \mathbb{K}^{k} \rightarrow \mathbb{K}^{(n-k) \times k}
$$

в случае $\Gamma=\nabla \psi$.

Teopema 4.6.

(1) Пусть $\psi \in C^{2}\left(\mathbb{K}^{n}\right)$. Слоение уровнями $\nabla \psi$ задано отображением $R: \mathbb{K}^{k} \rightarrow$ $\mathbb{K}^{(n-k) \times k}$ в окрестности нуля $U \times V \subset \mathbb{K}^{k} \times \mathbb{K}^{n-k}$, m.e.

$$
\nabla \psi(h+\lambda R(h), \lambda)=\nabla \psi(h, 0), \quad \forall h \in U \cap \Omega_{h}^{(k)}, \quad \lambda \in V,
$$

тогда и только тогда, когда

$$
D^{2} \psi(h+\lambda R(h), \lambda)\left(\begin{array}{c}
R^{T}(h) \\
I_{n-k}
\end{array}\right)=0 \in \mathbb{K}^{n \times(n-k)}, \quad \forall h \in U \cap \Omega_{h}^{(k)}, \quad \lambda \in V .
$$

В этом случае $\psi$ принимает вид (4.10) $с$

$$
\phi(h)=\psi(h, 0), \quad \chi_{k+l}(h)=\frac{\partial \psi}{\partial h_{k+l}}(h, 0), \quad l=1, \ldots, n-k .
$$

Если $\psi \in C^{3}\left(\mathbb{K}^{n}\right)$, то $\phi$ и $\chi_{k+l}$ удовлетворяют уравнениям (4.11), (4.12) ${ }_{8} \Omega_{h}^{(k)}$.

(2) Пусть функщия $\psi$ определена с помощью

$$
\begin{aligned}
\psi(h+\lambda R(h), \lambda) & =\phi(h)+\left\langle\lambda, \nabla \psi(h, 0)\left(\begin{array}{c}
R^{T}(h) \\
I_{n-k}
\end{array}\right)\right\rangle \\
& =\phi(h)+\sum_{l=1}^{n-k} \lambda_{l}\left[\sum_{j=1}^{k} \partial_{j} \phi(h) R_{l}^{j}(h)+\chi_{k+l}(h)\right],
\end{aligned}
$$

где $\phi \in C^{3}\left(\mathbb{K}^{n}\right)$ - решение системь из $\frac{1}{2}(k-1) k(n-k)$ дифференциальных уравнений в частных производных

(4.11)

$\sum_{i=1}^{k} \frac{\partial^{2} \phi}{\partial h_{i} \partial h_{j}} \frac{\partial R_{l}^{i}}{\partial h_{m}}-\sum_{i=1}^{k} \frac{\partial^{2} \phi}{\partial h_{i} \partial h_{m}} \frac{\partial R_{l}^{i}}{\partial h_{j}}=0, \quad 1 \leqslant m<j \leqslant k, 1 \leqslant l \leqslant n-k$,

и функции $\chi_{k+l} \in C^{2}\left(\mathbb{K}^{n}\right), l=1, \ldots, n-k$, удовлетворяют

$$
\partial_{j} \chi_{k+l}(h)+\sum_{i=1}^{k} \partial_{j} \partial_{i} \phi(h) R_{l}^{i}(h)=0, \quad 1 \leqslant j \leqslant k .
$$

Тогда $\psi$ удовлетворяет условию факторизации (4.8) локально в $h, \lambda, \partial л я$ которых выполнено неравенство

$$
\operatorname{det}\left(I_{k}+\sum_{l=1}^{n-k} \lambda_{l} D R_{l}(h)\right) \neq 0
$$


ДокАЗАТЕЛЬСтво. (1) Дифференцируя (4.8) по $\lambda$, получаем

$$
\begin{aligned}
\frac{\partial}{\partial \lambda_{i}} & \partial_{j} \psi(h+\lambda R(h), \lambda) \\
& =\sum_{m=1}^{k} \partial_{m} \partial_{j} \psi(h+\lambda R(h), \lambda) R_{i}^{m}(h)+\partial_{k+i} \partial_{j} \psi(h+\lambda R(h), \lambda) \\
& =\nabla \partial_{j} \psi(h+\lambda R(h), \lambda)\left(\begin{array}{c}
R_{i}^{T}(h) \\
\delta_{j, k+i}
\end{array}\right) .
\end{aligned}
$$

Из (4.8) вытекает, что выражение в (4.14) равно нулю. Так как это верно для всех $1 \leqslant j \leqslant n$ и $1 \leqslant i \leqslant n-k$, получаем (4.9). Теперь предположим, что вьполнено (4.9). Дифференцируя $\partial_{j} \psi(h+\lambda R(h), \lambda)$ с $1 \leqslant j \leqslant n$ по $\lambda_{i}$, получаем (4.14). В матричной записи по $i, j$ это значит

$$
\nabla_{\lambda} \nabla \psi(h+\lambda R(h), \lambda)=D^{2} \psi(h+\lambda R(h), \lambda)\left(\begin{array}{c}
R^{T}(h) \\
I_{n-k}
\end{array}\right)
$$

где последнее произведение равно нулю по (4.9). Поэтому

$$
\nabla \psi(h+\lambda R(h), \lambda)=\operatorname{const}(h)=\nabla \psi(h, 0),
$$

что соответствует (4.8). Дифференцируя $\psi(h+\lambda R(h), \lambda)$ по $\lambda$, получаем

$$
\nabla_{\lambda} \psi(h+\lambda R(h), \lambda)=\nabla \psi(h+\lambda R(h), \lambda)\left(\begin{array}{c}
R^{T}(h) \\
I_{n-k}
\end{array}\right)=\nabla \psi(h, 0)\left(\begin{array}{c}
R^{T}(h) \\
I_{n-k}
\end{array}\right)
$$

где последнее равенство следует из (4.8). Таким образом, $\psi$ имеет вид (4.10).

Теперь докажем (4.11). Записьвая (4.9) в скалярной форме и подставляя $\lambda=0$ для $1 \leqslant j \leqslant k$, имеем

$$
\sum_{i=1}^{k} \partial_{i} \partial_{j} \phi(h) R_{l}^{i}(h)+\partial_{k+l} \partial_{j} \psi(h, 0)=0, \quad 1 \leqslant l \leqslant n-k,
$$

что соответствует уравнениям (4.12), так как $\psi \in C^{2}\left(\mathbb{K}^{n}\right)$ и $\partial_{j} \partial_{k+l} \psi=\partial_{k+l} \partial_{j} \psi$. Тождества $\partial_{m} \partial_{j} \chi_{k+l}=\partial_{j} \partial_{m} \chi_{k+l}$ для $\psi \in C^{3}\left(\mathbb{K}^{n}\right)$ и (4.12) влекут

$$
\frac{\partial}{\partial h_{m}}\left(\sum_{i=1}^{k} \partial_{i} \partial_{j} \phi(h) R_{l}^{i}(h)\right)=\frac{\partial}{\partial h_{j}}\left(\sum_{i=1}^{k} \partial_{i} \partial_{m} \phi(h) R_{l}^{i}(h)\right) .
$$

Так как $\phi \in C^{3}$, из этих уравнений вытекает (4.11). 
(2) Определим $\psi$ как в (4.10). Сразу заметим, что уравнения (4.11) гарантируют существование функций $\chi_{k+l} \in C^{2}\left(\mathbb{K}^{n}\right)$, удовлетворяющих (4.12). Дифференцируя (4.10) по $h_{m}$ для всех $1 \leqslant m \leqslant k$, получаем

$$
\begin{aligned}
\partial_{m} \psi & (h+\lambda R(h), \lambda)\left(1+\lambda \partial_{m} R^{m}(h)\right)+\sum_{1 \leqslant i \leqslant k, i \neq m} \partial_{i} \psi(h+\lambda R(h), \lambda)\left(\lambda \partial_{m} R^{i}(h)\right) \\
& =\frac{\partial}{\partial h_{m}} \psi(h+\lambda R(h), \lambda) \\
& =\partial_{m} \phi(h)+\sum_{l=1}^{n-k} \lambda_{l}\left[\sum_{j=1}^{k}\left(\partial_{m} \partial_{j} \phi(h) R_{l}^{j}(h)+\partial_{j} \phi(h) \partial_{m} R_{l}^{j}(h)\right)+\partial_{m} \chi_{k+l}(h)\right] \\
& =\partial_{m} \phi(h)+\sum_{l=1}^{n-k} \lambda_{l}\left[\sum_{j=1}^{k} \partial_{j} \phi(h) \partial_{m} R_{l}^{j}(h)\right],
\end{aligned}
$$

где мы использовали определения (4.10) и (4.12). В матричных обозначениях,

$$
\nabla_{1}^{k} \psi(h+\lambda R(h), \lambda)\left(I_{k}+\sum_{l=1}^{n-k} \lambda_{l} D R_{l}(h)\right)=\nabla_{1}^{k} \psi(h, 0)\left(I_{k}+\sum_{l=1}^{n-k} \lambda_{l} D R_{l}(h)\right),
$$

и при $h, \lambda$, удовлетворяюших (4.13), получаем $\partial_{j} \psi(h+\lambda R(h), \lambda)=\partial_{j} \psi(h, 0), 1 \leqslant j \leqslant k$, что не зависит от $\lambda$. Для оставшихся компонент $\nabla \psi$ дифференцируем (4.10) по $\lambda_{l}$ и получаем

$$
\begin{aligned}
& \sum_{j=1}^{k} \partial_{j} \psi(h+\lambda R(h), \lambda) R_{l}^{j}(h)+\partial_{k+l} \psi(h+\lambda R(h), \lambda) \\
& \quad=\frac{\partial}{\partial \lambda_{l}} \psi(h+\lambda R(h), \lambda)=\sum_{j=1}^{k} \partial_{j} \phi(h) R_{l}^{j}(h)+\chi_{k+l}(h),
\end{aligned}
$$

где последнее равенство вытекает из определения (4.10). Это влечет

$$
\partial_{k+l} \psi(h+\lambda R(h), \lambda)=\chi_{k+l}(h)
$$

ввиду только что доказанного условия факторизации для $1 \leqslant j \leqslant k$. Доказательство закончено.

Из теоремы 4.6 следует, что слоение является слоением градиентного типа, если удовлетворена система уравнений (4.11), и мы рассматриваем (4.12) как определение функций $\chi_{k+l}$. Выражение (4.11) в матричной форме принимает вид

$$
\partial_{m} R_{l}(h)\left(D^{2} \phi(h)\right)^{j}=\partial_{j} R_{l}(h)\left(D^{2} \phi(h)\right)^{m}, \quad 1 \leqslant l \leqslant n-k,
$$

для каждой строки $R_{l}$ матрищы $R$, где $\left(D^{2} \phi(h)\right)^{j}$ - строка с номером $j$ матрицы $D^{2} \phi(h)$. Обозначая через $\mathbb{K}_{\mathrm{symm}}^{k \times k}$ пространство симметричных $k \times k$ матриц, заключаем, что справедливо 
СЛЕДСТВИЕ 4.7. Условие (4.11) әквивалентно условию

$$
D R_{l}(h)^{T} D^{2} \phi(h)=\left(\begin{array}{c}
\partial_{1} R_{l}(h) \\
\vdots \\
\partial_{k} R_{l}(h)
\end{array}\right) D^{2} \phi(h) \in \mathbb{K}_{\text {symm }}^{k \times k}, \quad \forall h \in \Omega_{h}^{(k)}, \quad 1 \leqslant l \leqslant n-k .
$$

Отметим, что в наших обозначениях $\partial_{m} R_{l}(h)$ означает столбец с номером $m$ матрищы $D R_{l}(h)$ и строку с номером $m$ матрицы $D R_{l}(h)^{T}$, поэтому транспозиция необходима.

ЗАмЕчАниЕ 4.8. Если вместо градиентного типа (4.7) мы предположим, что матрищы $D \Gamma$ и $D \gamma$ квадратны и симметричны, после возможного удаления или добавления линейно зависимых строк, то условие симметричности следствия 4.7 следует из уравнения (4.3) леммы 4.1 с помощью умножения слева на матрицу $\left(I_{k}+\sum_{l} \lambda_{l} D R_{l}(h)\right)^{T}$ и использования симметричности матриц $D \Gamma$ и $D \gamma$. Однако, матрища $D R(h)^{T} D \gamma(h)$ в примерах раздела 4.1 не обязана быть симметричной, в отличие от следствия 4.7 .

4.3. Восстановление фазовой функции. Функция $\psi$ в теореме $4.6,(2)$, очевидно, зависит от особенностей слоения $R$. В случае $\mathbb{K}=\mathbb{R}$ через $C^{m}(U)$ с $1 \leqslant m \leqslant \infty$ обозначим стандартное пространство функций на $U$, непрерьвно дифференцируемых $m$ раз, и пространство аналитических функций с $m=\omega$. В комплексном случае $\mathbb{K}=\mathbb{C}$ все эти пространства совпадают с пространством голоморфных функций на $U$.

Пусть $\Omega_{0}$ - множество точек $\Omega$, удовлетворяющих условию (4.13):

$$
\Omega_{0}=\left\{(h, \lambda) \in \Omega \cap\left(\mathbb{K}^{k} \times \mathbb{K}^{n-k}\right): \operatorname{det}\left(I_{k}+\sum_{l=1}^{n-k} \lambda_{l} D R_{l}(h)\right) \neq 0\right\} .
$$

Предположим, что слоение $R$ принадлежит классу $C^{m}$ в открытом подмножестве $\Omega^{a}$ множества $\Omega_{h}, \phi$ принадлежит $C^{m+1}(\Omega)$ и $\chi_{k+l} \in C^{m}(\Omega)$. Тогда отображение

$$
\Phi: \Omega \cap\left(\mathbb{K}^{k} \times \mathbb{K}^{n-k}\right) \ni(h, \lambda) \mapsto\left(\Phi_{1}(h, \lambda), \Phi_{2}(h, \lambda)\right) \in \mathbb{K}^{k} \times \mathbb{K}^{n-k}
$$

с компонентами

$$
\begin{aligned}
& \Phi_{1}(h, \lambda)=h+\sum_{l=1}^{n-k} \lambda_{l} R_{l}(h), \\
& \Phi_{2}(h, \lambda)=\lambda
\end{aligned}
$$

принадлежит $C^{m}$ на $\pi_{h}^{-1}\left(\Omega^{a}\right) \cap \Omega$, и его якобиан имеет вид

$$
\operatorname{det} D \Phi(h, \lambda)=\operatorname{det}\left(\begin{array}{cc}
I_{k}+\lambda D R(h) & R(h) \\
0 & I_{n-k}
\end{array}\right)=\operatorname{det}\left(I_{k}+\sum_{l=1}^{n-k} \lambda_{l} D R_{l}(h)\right),
$$

что отлично от нуля при $(h, \lambda) \in \Omega_{0}$. По теореме о неявной функции отображение $\Phi^{-1}$ принадлежит $C^{m}$ в $\Phi\left(\pi_{h}^{-1}\left(\Omega^{a}\right) \cap \Omega_{0}\right)$. Из утверждения теоремы $4.6,(2)$, вытекает, что на $\Omega_{0}$ функция $\psi$ удовлетворяет

$$
(\psi \circ \Phi)(h, \lambda)=\phi(h)+\sum_{j=1}^{k} \partial_{j} \phi(h)\left(\Phi_{1}(h, \lambda)-h\right)^{j}+\sum_{l=1}^{n-k} \lambda_{l} \chi_{k+l}(h)
$$

и, следовательно, $\psi \in C^{m}$ на $\Phi\left(\pi_{h}^{-1}\left(\Omega^{a}\right) \cap \Omega_{0}\right) \subset \Phi(\Omega)$. Таким образом, получаем 
УТВЕРЖДЕНИЕ 4.9. Пусть $R: \Omega^{a} \subset \Omega_{h} \subset \mathbb{K}^{k} \rightarrow \mathbb{K}^{(n-k) \times k}, \Omega^{a}-$ открытое подмножество $\Omega_{h}, 3 \leqslant m \leqslant \infty$ или $m=\omega, R \in C^{m}\left(\Omega^{a}\right), \phi \in C^{m+1}\left(\Omega_{h}\right)$, $\chi_{k+l} \in C^{m}\left(\Omega_{h}\right)$. Пусть $\Omega_{0}$ задано формулой (4.15) и $\Phi$ задана формулой (4.16). Тогда функиия $\psi$ из (4.10) принадлежит $C^{m}{ }_{\text {в }} \Phi\left(\pi_{h}^{-1}\left(\Omega^{a}\right) \cap \Omega_{0}\right) \subset \Phi(\Omega)$.

Предположим теперь, что множества $\pi_{h}^{-1}\left(\Omega^{a}\right), \Omega$ и $\Omega_{0}$ локально совпадают, и обозначим через $U$ их открытое пересечение. В частности, предположение верно в случае $m=\omega$, если слоение локально аналитично и задано аналитическим отображением $R$, для которого условие (4.13) локально вьполнено. Как следует из утверждения 4.3, это условие может быть вьполнено также глобально, если все матрицы $D R_{l}$ нильпотентны. По теореме 4.6 система (4.11) дифференциальных уравнений в частных производных дает достаточное условие на существование функции $\psi$, для которой слоение уровнями $\nabla \psi$ локально задано с помощью $R$. Из утверждения 4.9 следует, что $\psi$ аналитична в $\Phi(U)$.

Однако нет гарантии, что решение $\phi$ системы (4.11) дифференциальных уравнений второго порядка в частных производных принадлежит $C^{m}$. В комплексном случае $(\mathbb{K}=\mathbb{C})$ можно использовать теорему Коши-Ковалевской и получить аналитическое решение $\phi$ в окрестности точки, в которой не все коэффициенты при производных наивысших порядков равны нулю. В этом случае получаем аналитическое решение даже для задачи Коши, задавая $\phi$ на некоторой нехарактеристической гиперповерхности.

Интересно было бы получить обобшения утверждения 4.9. В общем, при слоении, заданном отображением $R \in C^{m}$, возможно ли восстановить функцию $\psi$, для которой слоение уровнями $\nabla \psi$ локально совпадает со слоением, задаваемым $R$ ? Если такая функция $\psi$ существует, когда можно гарантировать ее гладкость? Возникает частный случай при аналитическом слоении $R$. В соответствии с замечаниями вьше о теореме Коши-Ковалевской можно сделать вьвод об аналитичности функций $\phi$ и $\chi$. Однако функция $\psi$, получаемая по формулам теоремы 4.6, может быть комплекснозначной даже на вешественной области. Интересно было бы получить критерии, при которых полученная по теореме 4.6 аналитическая функция $\psi$ принимает вешественные значения на вешественной области. Или, по крайней мере, когда мнимая часть $\psi$ имеет постоянньй знак на вещественной области. В последнем случае $\psi$ приводит к допустимой комплекснозначной фазовой функции (см. раздел 5.3 и замечание 5.2).

В общем, подход, предложенный теоремой 4.6, сводит поставленные выше вопросы к анализу системы дифференциальных уравнений (4.11). Свойства решений такой системы зависят от ее типа, определяемого знаком ее детерминанта, которьй в свою очередь выражается в терминах отображения $R$. В целом, если слоение достаточно гладко, то коэффициенты системы (4.11) также достаточно гладки. В этом случае некоторые свойства можно найти в [27], [13]. Для гиперболических уравнений см. [25]. Для слоений с сушественными особенностями коэффициенты системы (4.11) мероморфны и заданы рациональными функциями.

В случае трехмерного пространства с $n=3$ система состоит только из одного уравнения и анализ упрощается. Предположим теперь, что $n=3$ и $k=2$. По теореме 3.10 для случая $\mathbb{K}=\mathbb{C}$ слоения с аффинными слоями всюду аналитичны. Поэтому предположим также гладкость и в вещественном случае $\mathbb{K}=\mathbb{R}$. Система (4.11) для $\phi: \mathbb{K}^{2} \rightarrow \mathbb{K}$ принимает вид 


$$
\frac{\partial^{2} \phi}{\partial h_{1}^{2}} \frac{\partial R_{1}}{\partial h_{2}}+\frac{\partial^{2} \phi}{\partial h_{1} \partial h_{2}}\left[\frac{\partial R_{2}}{\partial h_{2}}-\frac{\partial R_{1}}{\partial h_{1}}\right]-\frac{\partial^{2} \phi}{\partial h_{2}^{2}} \frac{\partial R_{2}}{\partial h_{1}}=0 .
$$

УТВЕРЖДЕНИЕ 4.10.

(1) Если матрица $D R(h)$ не имеет вещественных собственных значений, то уравнение (4.17) әллиптического типа. В этом случае слоение продолжимо глобально по $\lambda \in \mathbb{R}$ локально равномерно по $h$.

(2) Eсли матрица $D R(h)$ имеет вещественное ненулевое собственное значение, то определитель матричы $D^{2} \phi(h)$ равен нулю.

(3) Уравнение (4.17) есть уравнение әллиптического, параболического, гиперболического типа, если собственные значения матриць $D R(h)$ не вещественны, вещественны и равны, вещественны и различны соответственно.

(4) Пусть $\psi \in C^{\omega}\left(\mathbb{R}^{3}\right), h \in \mathbb{R}^{2}$. Если определитель матриць $D^{2} \phi(h)$ равен нулю, то существует окрестность $U$ точки $h$ такая, что уравнение (4.17) параболического типа в $U$. Такой же вывод справедлив, если $\psi \in C^{3}\left(\mathbb{R}^{3}\right)$ и слоение глобально продолжимо по $\lambda$ локально равномерно no $h$.

ДокАЗАтЕльство. (1) Если матрища $D R(h)$ не имеет вешественных собственных значений, то условие (4.13) выполнено для всех вешественных $\lambda$, откуда вытекает, что слоение продолжимо глобально по $\lambda$. Обозначим $R_{i j}(h)=\partial_{j} R_{i}(h)$. Уравнение для собственных значений матришы $D R(h)$ имеет вид $\operatorname{det}\left(\begin{array}{cc}R_{11}-\mu & R_{12} \\ R_{21} & R_{22}-\mu\end{array}\right)=0$. Дискриминант равен $d(h)=\left(R_{11}(h)+R_{22}(h)\right)^{2}-4 \operatorname{det} D R(h)$ и по нашему предположению $d(h)<0$. Тип уравнения $(4.17)$ задается знаком

$$
\operatorname{det}\left(\begin{array}{cc}
R_{12} & \frac{1}{2}\left[R_{22}-R_{11}\right] \\
\frac{1}{2}\left[R_{22}-R_{11}\right] & -R_{21}
\end{array}\right)
$$

т.е. $-R_{12} R_{21}-\frac{1}{4}\left(R_{22}-R_{11}\right)^{2}=-\frac{1}{4}\left(R_{22}+R_{11}\right)^{2}+\operatorname{det} D R=-d(h) / 4$. Это означает, что уравнение (4.17) эллиптического типа.

(2) Пусть $\mu \in \mathbb{R}, \mu \neq 0,-$ собственное число матрицы $D R(h): D R(h) v=\mu v$. Дифференцируя (4.8) по $h$, получаем

$$
\left(\begin{array}{cc}
\partial_{1}^{2} \psi(h+\lambda R(h), \lambda) & \partial_{1} \partial_{2} \psi(h+\lambda R(h), \lambda) \\
\partial_{2} \partial_{1} \psi(h+\lambda R(h), \lambda) & \partial_{2}^{2} \psi(h+\lambda R(h), \lambda)
\end{array}\right)(I+\lambda D R(h))=D^{2} \phi(h) .
$$

Применяя обе части к $v$ и подставляя $\lambda=-\mu^{-1}$, заключаем, что $D^{2} \phi(h) v=0$.

(3) Следует из рассуждения части (1).

(4) По непрерывности $D^{2} \phi(h)$ существует окрестность $U$ точки $h$ такая, что определитель матрицы $D^{2} \phi$ ненулевой в $U$. Рассуждая, как при доказательстве утверждения 4.3 , получаем, что матрица $D R$ нильпотентна в $U$. По части (2) получаем утверждение. Утверждение 4.10 доказано.

В комплексном случае $(\mathbb{K}=\mathbb{C})$ с голоморфным слоением $R$, если для $i=1$ или $i=2$ через $h_{i}$ обозначить переменную, для которой $\partial R_{i} / \partial h_{j}, j \neq i$, в (4.17) не обрашается 
в нуль при $h_{i}=0$, из теоремы Коши-Ковалевской вытекает, что уравнение (4.17) с голоморфными данньми Коши $\partial_{h_{i}}^{l} \phi, l<2$, локально разрешимо и решение единственно и голоморфно. В общем, если данные Коши голоморфны на некоторой поверхности $S$ в $\mathbb{C}^{2}$, нехарактеристической для (4.17), то сушествует единственное решение $\phi$ уравнения (4.17) локально в каждой точке $S$ ([24; Vol. I, Theorems IX.4.5-IX.4.7]).

В вешественном случае $(\mathbb{K}=\mathbb{R})$ гладкость решения уравнения (4.17) зависит от его типа. Для эллиптического типа имеем следующий результат.

TЕОрема 4.11. Пусть отображсение $R: U \subset \mathbb{R}^{2} \rightarrow \mathbb{R}^{2}$ гладкое (вещественно аналитическое), и предположим, что для всех $h \in U$ матрича $D R(h)$ не имеет вещественных собственных значений. Тогда существует гладкая (вещественно аналитическая) функиия $\psi: \Omega \subset \mathbb{R}^{3} \rightarrow \mathbb{R}$ такая, что слоение, задаваемое уровнями градиента $\nabla \psi$, задано с помощью $R$ в $U$.

ДокАЗАТЕЛЬСТво. Из утверждения 4.10 следует, что уравнение (4.17) эллиптического типа. Тогда его решения $\psi$ гладки (аналитичны), если оператор в (4.17) имеет гладкие (аналитические) коэффициенты. См. следствие 8.3.2 для $W F(\phi)$ и теорему 9.5.1 для $W F_{A}(\phi)$ в [24] соответственно. Также все $\chi_{k+l}$ гладки (аналитичны), и утверждение 4.9 завершает доказательство.

Обсудим вкратце случай, когда уравнение (4.17) строго гиперболического типа. Если отображение (слоение) $R$ задано на гладкой гиперповерхности $S$, не характеристической для (4.17), то решение $\phi$ принадлежит пространству Соболева $H^{(s)}$, если данные Коши лежат в пространстве Соболева $H^{(s)}$. Применяя теорему вложения для всех $s>0$, получаем, что решение $\phi$ гладко, если данные Коши гладки на $S$. Для аналитических слоений применим метод, описанньй в $[9 ; \mathrm{VI}]$. Пусть $\Omega$ - связное комплексно аналитическое многообразие некоторой размерности $p$. Через $\mathbb{D}(\Omega)$ обозначим алгебру линейных дифференциальных операторов в частных производных с коэффициентами, голоморфными в $\Omega$. Пусть $J$ - левый идеал в $\mathbb{D}(\Omega)$. Тогда $J$ называется голономным в $\Omega$, если для любого $x \in \Omega$ пересечение нулей в $\mathbb{C}^{p}$ главных символов $q^{x}(\theta)$ в точке $x$ операторов $Q \in J$ (в локальных координатах) содержит только нуль. В частности, голономность идеала, порожденного гиперболическим оператором $P$, позволяет свести задачу к обычному дифференциальному уравнению вдоль произвольной кривой в дополнении к комплексному бихарактеристическому конусу, соответствуюшему главному символу оператора $P$, и применить стандартные результаты об аналитическом продолжении для обыкновенных дифференциальных уравнений. Не приводя подробностей, отметим только, что из [9; VI-16, Theorem] вытекает

УТВЕРЖДЕНИЕ 4.12. Пусть отображсение $R$ аналитично в открытом множестве $\Omega_{0} \subset \mathbb{R}^{2}$, и пусть на $\Omega_{0}$ собственные значения матрицы $D R$ вещественны и различны. Через $\Omega$ обозначим комплексное расширение множества $\Omega_{0}$, и пусть $\phi$ - решение уравнения (4.17). Предположим также, что идеал $J=\{Q \in \mathbb{D}(\Omega): Q \phi=0\}$, порожсенный уравнением (4.17), голономен в $\Omega$. Тогда функиия $\phi$ вещественно аналитична в $\Omega_{0}$ и допускает голоморфное продолжение $\phi_{\gamma}$ вдоль любой кривой $\gamma$ в $\Omega$, начинающейся в $\Omega_{0}$. Голоморфное продолжение $\phi_{\gamma}$ также удовлетворяет уравнениям $Q \phi_{\gamma}=0$ для всех $Q \in J$.

Приведем несколько следствий теоремы 4.6 для вещественного случая $\mathbb{K}=\mathbb{R}$ и произвольного $k$. 


\section{СЛЕДСТВИЕ 4.13.}

(1) Пусть определитель матрищъ $\left.D^{2} \phi(h)\right|_{h=h_{0}}$ равен нулю. Предположим, что $R\left(h_{j}\right) \rightarrow R_{0}$ при $h_{j} \rightarrow h_{0}, h_{j} \in \Omega_{h}^{(k)}$. Если определитель матрицы

$$
\left(\begin{array}{ccc}
\partial_{1}^{2} \psi\left(h_{0}+\lambda R_{0}, \lambda\right) & \ldots & \partial_{1} \partial_{k} \psi\left(h_{0}+\lambda R_{0}, \lambda\right) \\
\ldots \ldots \ldots \ldots \ldots \ldots \ldots \ldots \ldots \ldots \ldots \ldots \ldots \ldots \ldots \ldots \ldots \\
\partial_{k} \partial_{1} \psi\left(h_{0}+\lambda R_{0}, \lambda\right) & \ldots & \partial_{k}^{2} \psi\left(h_{0}+\lambda R_{0}, \lambda\right)
\end{array}\right)
$$

ненулевой для некоторого $\lambda \in \mathbb{R}^{n-k}$, то существует предел

$$
A=\lim _{j \rightarrow \infty} \sum_{l=1}^{n-k} \lambda_{l} D R_{l}\left(h_{j}\right)
$$

$u(-1)$ - собственное значение матрицы $A$.

(2) Если для $h \in \Omega_{h}$ существует $\lambda \in \mathbb{R}^{n-k}$ maкое, что $(h, \lambda) \in \Omega u-1$ есть собственное значение матрицы $\sum_{l=1}^{n-k} \lambda_{l} D R_{l}(h)$, то определитель матрици $D^{2} \phi(h)$ равен нулю.

(3) Если для глобального слоения хотя би одна из матрии $D R_{j}(h), 1 \leqslant j \leqslant$ $n-k$, имеет вещественное ненулевое собственное значение, то определитель матриць $D^{2} \phi(h)$ равен нулю.

ДоказАтельство. (1) Сразу заметим, что при $\lambda=0$ матрица (4.18) равна $D^{2} \phi\left(h_{0}\right)$ и $\lambda \neq 0$, так как определитель матрищы (4.18) не нуль. Дифференцируя (4.8) по $h$, получаем

(4.19)

$$
\left(\begin{array}{ccc}
\partial_{1}^{2} \psi(h+\lambda R(h), \lambda) & \ldots & \partial_{1} \partial_{k} \psi(h+\lambda R(h), \lambda) \\
\ldots \ldots \ldots \ldots \ldots \ldots \ldots \ldots \ldots \ldots \ldots \ldots \ldots \ldots \ldots \ldots \ldots \\
\partial_{k} \partial_{1} \psi(h+\lambda R(h), \lambda) & \ldots & \partial_{k}^{2} \psi(h+\lambda R(h), \lambda)
\end{array}\right)\left(I_{k}+\sum_{l=1}^{n-k} \lambda_{l} D R_{l}(h)\right)=D^{2} \phi(h) .
$$

Значит, существует матричньй предел у $I_{k}+\sum_{l=1}^{n-k} \lambda_{l} D R_{l}\left(h_{j}\right)$ и у $\sum_{l=1}^{n-k} \lambda_{l} D R_{l}\left(h_{j}\right)$, так как $\lambda \neq 0$. Собственное значение -1 получаем из $\operatorname{det}\left(I_{k}+A\right)=0$.

(3) Пусть $\mu \in \mathbb{R}$ - собственное значение матрицы $D R_{j}(h)$ для некоторого $1 \leqslant j \leqslant$ $n-k: D R_{j}(h) v=\mu v$. Дифференпируя (4.8) по $h$, получаем

$$
\left(\partial_{i} \partial_{j} \psi(h+\lambda R(h), \lambda)\right)_{1 \leqslant i, j \leqslant k}\left(I_{k}+\sum_{l=1}^{n-k} \lambda_{l} D R_{l}(h)\right)=D^{2} \phi(h) .
$$

Применяя обе части к $v$ и подставляя $\lambda_{j}=-\mu^{-1}$ и $\lambda_{l}=0$ для $l \neq j$, получаем $D^{2} \phi(h) v=0$. Доказательство (2) аналогично.

Наконец, сделаем несколько замечаний в случае $k=n-1$. В этом случае имеем только одну матрицу $D R_{1}$. Если при некотором $h$ матрица $D R_{1}(h)$ не имеет вещественных собственных значений, то слоение продолжимо глобально по $\lambda \in \mathbb{R}$ локально равномерно по $h$. 
СЛЕДСТВИЕ 4.14. Пусть $k=n-1$ и предположим, что определитель матрицв $\left.D^{2} \phi(h)\right|_{h=h_{0}}$ равен нулю. Пусть $R_{0}=\lim _{j} R\left(h_{j}\right)$ при $h_{j} \rightarrow h_{0}, h_{j} \in \Omega_{h}^{(k)}$. Тогда имеем

$$
\operatorname{det}\left(\begin{array}{ccc}
\partial_{1}^{2} \psi\left(h+\lambda R_{0}, \lambda\right) & \ldots & \partial_{1} \partial_{n-1} \psi\left(h+\lambda R_{0}, \lambda\right) \\
\ldots \ldots \ldots \ldots \ldots \ldots \ldots \ldots \ldots \ldots \ldots \ldots \ldots \ldots \ldots \\
\partial_{n-1} \partial_{1} \psi\left(h+\lambda R_{0}, \lambda\right) & \ldots & \partial_{n-1}^{2} \psi\left(h+\lambda R_{0}, \lambda\right)
\end{array}\right) \equiv 0
$$

для всех $\lambda$ в некоторой окрестности нуля. Более того, если существует предел $A=\lim _{j} D R\left(h_{j}\right)$, то тождество (4.20) виполнено для всех $|\lambda|<\rho(A)^{-1}$, әде $\rho(A)$ - спектральный радиус матрицы $A$. Если предел $\lim _{j} D R\left(h_{j}\right)$ не существует, то тождество (4.20) верно для всех $\lambda$, для которых оно определено.

ДокАЗАТЕЛЬСТво. Если тождество (4.20) не выполнено, то по второй части следствия 4.13 существует предел $A=\lim _{j} D R\left(h_{j}\right)$ и -1 есть собственное значение матрипы $\lambda A$. Очевидно, это не верно при $|\lambda|<\rho(A)^{-1}$. Последнее утверждение следует из (4.19), если использовать тот факт, что $\operatorname{det}\left(I_{n-1}+\mu A\right)=0$.

Отметим, что можно выбрать $\lambda$ в следствии 4.14 независимо от $R_{0}$, так как зависимость $\lambda$ от $R_{0}$ непрерывна и $R_{0}$ соответствует отображению со значениями в компактном грассманиане $\mathbb{G}_{1}\left(\mathbb{C}^{n}\right)$. В комплексных обозначениях раздела 3 утверждение следствия 4.14 имеет вид

$$
\left(h_{0}, 0\right) \in \Omega \backslash \Omega^{(n-1)} \Rightarrow \bar{\varkappa}\left(h_{0}, 0\right) \cap \Omega \subset \Omega \backslash \Omega^{(n-1)},
$$

что соответствует лемме 3.25 .

4.4. Сушествование сингулярных слоений градиентного типа. Из теоремы 3.9 и результатов раздела 3 видно, что минимальная размерность пространства над $\mathbb{C}$, в котором могут существовать слоения с существенными особенностями, это $n=4 \mathrm{c} k=3$. Продемонстрируем, что в $\mathbb{C}^{4}$ сушествует слоение прямыми, соответствуюшее отображению градиентного типа $\Gamma=\nabla \psi$ с аналитической (даже полиномиальной) функцией $\psi$.

ПрИмеР 4.15. Пусть

$$
\psi\left(\xi_{1}, \xi_{2}, \xi_{3}, \xi_{4}\right)=\xi_{1} \xi_{2}^{2}+\left(\xi_{3}-\xi_{2} \xi_{4}\right)^{2} .
$$

Гессиан функции $\psi$ имеет вид

$$
D^{2} \psi(\xi)=\left(\begin{array}{cccc}
0 & 2 \xi_{2} & 0 & 0 \\
2 \xi_{2} & 2 \xi_{1}+2 \xi_{4}^{2} & -2 \xi_{4} & 4 \xi_{2} \xi_{4}-2 \xi_{3} \\
0 & -2 \xi_{4} & 2 & -2 \xi_{2} \\
0 & 4 \xi_{2} \xi_{4}-2 \xi_{3} & -2 \xi_{2} & 2 \xi_{2}^{2}
\end{array}\right)
$$

c $k=3$. Более того,

$$
\left.\operatorname{rank} D^{2} \psi\right|_{\xi_{2}=0}=2,\left.\quad \operatorname{rank} D^{2} \psi\right|_{\xi_{2}=\xi_{3}=0}=2 \quad \text { и }\left.\quad \operatorname{rank} D^{2} \psi\right|_{\xi_{1}=\xi_{2}=\xi_{3}=0}=1 .
$$

При $\xi_{2} \neq 0$ ядро матрицы $D^{2} \psi(\xi)$ одномерно,

$$
\operatorname{ker} D^{2} \psi(\xi)=\left\langle\left(\frac{\xi_{3}}{\xi_{2}}-\xi_{4}, 0, \xi_{2}, 1\right)\right\rangle \text {. }
$$

Следовательно, при $\lambda=\xi_{4}$ слоение, определяемое отображением $R$, имеет координаты $R^{1}(h)=h_{3} / h_{2}, R^{2}(h)=0, R^{3}(h)=h_{2}$. Функция $R^{1}$ имеет сушественную особенность при $h_{2}=h_{3}=0$ и $\Omega^{\operatorname{sing}}=\left\{\xi_{2}=\xi_{3}=0\right\}$. 
ПРИМЕР 4.16. Функции

$$
\psi\left(\xi_{1}, \xi_{2}, \xi_{3}, \xi_{4}\right)=\xi_{1} \xi_{2}^{k}+\left(\xi_{3}-\xi_{2} \xi_{4}\right)^{m}
$$

$c k, m \geqslant 2$ приводят к слоениям, имеюшим сушественные особенностипри $\xi_{2}=\xi_{3}=0$. Слои заданы с помошью

$$
\operatorname{ker} D^{2} \psi(\xi)=\left\langle\left(\frac{m}{k} \frac{\left(\xi_{3}-\xi_{2} \xi_{4}\right)^{m-1}}{\xi_{2}^{k-1}}, 0, \xi_{2}, 1\right)\right\rangle
$$

и

$$
R^{1}(h)=\frac{m}{k} \frac{h_{3}^{m-1}}{h_{2}^{k-1}} .
$$

ПРИМЕР 4.17. В $n$-мерном пространстве $\xi=\left(x_{1}, \ldots, x_{n-3}, y, z, w\right)$ определим

$$
\psi(\xi)=y^{2} \sum_{i=1}^{n-3} x_{i}+(z-y w)^{2}
$$

Уровни градиента $\nabla \psi$ имеют размерность $n-3$ и, значит, $k=3$. Можно проверить, что $\operatorname{dim} \Omega^{\operatorname{sing}}=n-2$ (что также следует из оценок на размерность в теореме $3.9 \mathrm{c}$ $k=3$ ).

ПРИМЕР 4.18. Рассмотрим функцию

$$
\psi(x, y, z, v, w, s, t)=x y^{2}+s v^{2}+(z-y w-v t)^{2} .
$$

Максимальный ранг гессиана $D^{2} \psi$ равен $k=5$, и его ядро натянуто на векторы

$$
\left(\frac{z-y w-v t}{y}, 0,0, y, 1,0,0\right), \quad\left(0,0,0, v, 0, \frac{z-y w-v t}{v}, 1\right)
$$

На множестве $y=0, z-v t=0$ первьй вектор в ядре имеет существенную особенность, в то время как второй вектор непрерывен при $v \neq 0$. Поэтому $\{y=0, z=v t\} \subset \Omega^{\operatorname{sing}}$ и $\operatorname{dim} \Omega^{\operatorname{sing}}=n-2$ по теореме 3.23 .

Приведенные примеры демонстрируют особенности слоений с различными размерностями слоев. С другой стороны, размерность $\Omega^{\operatorname{sing}}$ во всех примерах равна $n-2$. В примерах 4.15, 4.16 это необходимо, так как при $n=4$ из теоремы 3.10 вытекает, что $k=3$, и поэтому по теореме $3.9,(1)$, имеем $\operatorname{dim} \Omega^{\operatorname{sing}}=n-2$. В этом случае вьполнено также и более сильное утверждение 3.30. В примерах 4.17 и 4.18 с различными размерностями слоев все еше имеем $\operatorname{dim} \Omega^{\operatorname{sing}}=n-2$. Однако, приведенные конструкции основаны на одинаковой идее построения особенности. Было бы интересно исследовать, действительно ли условие $\operatorname{dim} \Omega^{\operatorname{sing}}=n-2$ необходимо для слоений градиентного типа. 


\section{5. Дальнейшие оценки для интегральных операторов Фурье}

5.1. Комплексификация. В этом разделе мы вкратце опишем комплексификацию рассмотренных выше множеств. В комплексной области мы сможем применить результаты разделов 3 и 4 . Для вешественного аналитического многообразия $M$ через $\widetilde{M}$ обозначим его комплексное расширение (комплексификацию). Пусть $\Lambda$ - вещественно аналитическое коническое лагранжево подмногообразие в $T^{*} M \backslash 0$. По теореме 2.3 производящая функция $\phi$ для локализации $\Lambda_{\phi}$ многообразия $\Lambda$ также вешественно аналитична. Комплексификацию $\widetilde{\Lambda}$ многообразия $\Lambda$ в комплексификации кокасательного расслоения $\widetilde{T^{*} M}$ можно рассматривать как комплексно аналитическое коническое лагранжево подмногообразие в $T^{*} \widetilde{M} \backslash 0$.

Предположим, что переменные $\widetilde{\xi}$ можно взять в качестве локальных координат для $\widetilde{\Lambda}$ в $\left(\widetilde{m}_{0}, \widetilde{\xi}_{0}\right) \in \widetilde{\Lambda}$. Тогда в окрестности точки $\left(\widetilde{m}_{0}, \widetilde{\xi}_{0}\right)$ многообразие $\widetilde{\Lambda}$ имеет вид

$$
\widetilde{m}_{i}=H_{i}(\widetilde{\xi}), \quad 1 \leqslant i \leqslant \operatorname{dim} \widetilde{M},
$$

где $H_{i}$ - голоморфные функции, вешественнозначные для вешественных $\widetilde{\xi}$. Лагранжево многообразие $\widetilde{\Lambda}$ инволютивно, и поэтому скобка Пуассона задает идеал на $\widetilde{\Lambda}$, т.е. $\{f, g\}=0$ на $\widetilde{\Lambda}$, если $f=0$ и $g=0$ на $\widetilde{\Lambda}$ (см. [5; 5.4.6]). В частности,

$$
\left.\left\{\widetilde{m}_{i}-H_{i}(\widetilde{\xi}), \widetilde{m}_{j}-H_{j}(\widetilde{\xi})\right\}\right|_{\widetilde{\Lambda}}=0
$$

для всех $i, j$ и, следовательно,

$$
\frac{\partial H_{i}}{\partial \widetilde{\xi}_{j}}=\frac{\partial H_{j}}{\partial \widetilde{\xi}_{i}}, \quad 1 \leqslant i, j \leqslant \operatorname{dim} \widetilde{M}
$$

Это значит, что все смешанные производные формы $\sum_{i} H_{i}(\widetilde{\xi}) d \widetilde{\xi}_{i}$ сокрашаются и она должна быть замкнутой и, следовательно, точной. Таким образом, существует голоморфная функция $H$ с $\partial H / \partial \widetilde{\xi}_{i}=H_{i}$ и $\widetilde{\Lambda}$ локально принимает вид $\{(\nabla H(\widetilde{\xi}), \widetilde{\xi})\}$. Такой же аргумент применим к $\Lambda$ в вешественной области, т.е. имеем локальную параметризацию $\Lambda$ с помошью $(\nabla H(\xi), \xi)$ с той же функцией $H$. Утверждение справедливо и в общем случае, когда переменных $\widetilde{\xi}$ не достаточно для задания локальной системы координат (см. [5; 5.6.4] для комплексного случая и [10; лемма 3.7.4] для вешественного).

Пусть $\pi$ - стандартная проекция с $T^{*} M$ на $M$ и $\widetilde{\pi}$ - ее комплексификация с $T^{*} \widetilde{M}$ на $\widetilde{M}$. Через $\Lambda^{(k)}$ обозначим открытое подмножество $\Lambda$, на котором достигается максимальньй ранг $k$ отображения $\left.d \pi\right|_{\Lambda}$. По теореме о неявной функции множество $\Sigma^{(k)}=\pi\left(\Lambda^{(k)}\right)$ - гладкое аналитическое $k$-мерное подмногообразие в $M$, содержашееся в $\Sigma=\pi(\Lambda)$. Имеем также $\Lambda^{(k)}=N^{*} \Sigma^{(k)}$. Пусть $\widetilde{\Sigma}^{(k)}$ - комплексное расширение $\Sigma^{(k)}$ в $\widetilde{M}, \widetilde{\Sigma}^{(k)}$ - гладкое аналитическое многообразие комплексной размерности $k$. Его конормальное расслоение $N^{*} \widetilde{\Sigma}^{(k)}-$ комплексно аналитическое лагранжево подмногообразие в $T^{*} \widetilde{M}$, содержашее $\Lambda^{(k)}$, где мы отождествляем $T^{*} \widetilde{M}$ и $\widetilde{T^{*} M}$. Через $\widetilde{\Lambda}^{(k)}$ обозначим комплексификацию $\Lambda^{(k)}$. Множества $\widetilde{\Lambda}^{(k)}$ и $N^{*} \widetilde{\Sigma}^{(k)}$ имеют комплексную размерность $n$ и локально совпадают. Таким образом, множество $N^{*} \widetilde{\Sigma}^{(k)}$ открыто в $\widetilde{\Lambda}$ и слои $\left.\widetilde{\pi}\right|_{\widetilde{\Lambda}^{(k)}}-$ линейные подпространства 
комплексной коразмерности $k$ кокасательных пространств многообразия $\widetilde{M}$. Его дополнение $\widetilde{\Lambda} \backslash \widetilde{\Lambda}^{(k)}$ аналитично и нигде не плотно.

Теперь применим описанную конструкцию к $M=X \times Y$, где $X$ и $Y$-вешественные аналитические многообразия. Пусть $\Lambda$ - однородное каноническое отношение, удовлетворяющее условию локального графика, и пусть $\phi-$ его производящая функция, как в (2.10) и (2.11). Тогда $\phi$ вешественно аналитична, и мы обозначим через $\widetilde{\phi}$ ее комплексное продолжение. Так как комплексное голоморфное продолжение единственно, $\widetilde{\phi}$-производящая функция для $\widetilde{\Lambda}$. Рассуждая, как в разделе 2.4 , заключаем, что слои отображения

$$
\Gamma: \widetilde{\xi} \mapsto \nabla_{\widetilde{\xi}} \widetilde{\phi}(\widetilde{y}, \widetilde{\xi})
$$

- аффинные подпространства $\mathbb{C}^{n}$ коразмерности $k$ для всех $(\widetilde{y}, \widetilde{\xi})$ в открытом плотном множестве, на котором максимальньй ранг $D_{\widetilde{\xi} \widetilde{\phi}}^{2} \widetilde{\phi}$ равен $n+k$. Таким образом, мы доказали следуюшую теорему.

Теорема 5.1. Пусть $X, Y$ - вещественно аналитические многообразия размерности $n$, и пусть $\Lambda$ - аналитическое однородное каноническое отношение из $T^{*} Y \backslash 0$ в $T^{*} X \backslash 0$. Пусть максимальный ранг $\left.d \pi_{X \times Y}\right|_{\Lambda}$ равен $n+k$. Пусть $\phi$ - локальная производящая функиия для $\Lambda$, как в (2.10), (2.11). Обозначим через $\widetilde{X}, \widetilde{Y}, \widetilde{\Lambda}, \widetilde{\phi}$ комплексные аналитические расширения $X, Y, \Lambda, \phi$. Тогда отображение (5.1) удовлетворяет условиям (P1)-(Р3) введения.

ЗАмЕЧАнИЕ 5.2. Пусть $X$ и $Y$ - гладкие многообразия без вешественной аналитичности. Через $\not$ обозначим почти аналитическое продолжение производящей функции $\phi$. Это означает, что ее сужение на вешественную область равно $\phi$ и $\bar{\partial} \widetilde{\phi}=0 \mathrm{c}$ бесконечной кратностью на вешественной области. Если $\operatorname{Im} \widetilde{\phi} \leqslant 0$, то такая теория интегральных операторов Фурье существует для почти аналитических продолжений (см. [33], [24], [12], [30]). В этом случае результаты раздела 3 применимы с точностью до неаналитической части, все производные которой обращаются в нуль.

5.2. Операторы, коммутирующие со сдвигами. Пусть $X, Y$-открытые множества в $\mathbb{R}^{n}$. Предположим, что оператор $T \in I^{\mu}(X, Y ; \Lambda)$ коммутирует со сдвигами. Это значит, что $T$ задается как свертка с некоторой обобшенной функцией $w$. Тогда функция $w$ должна быть распределением Фурье порядка $\mu$, задаваемьм некоторым коническим лагранжевым многообразием $C \subset T^{*} X$. Условие факторизации говорит, что ранг проекции $\pi: C \rightarrow \mathbb{R}^{n}$ не превосходит $k$ и равен $k$ в точках обшего положения, а сама проекция $\pi$ допускает факторизацию

$$
\pi=\alpha \circ \beta
$$

где $\beta$ задает расслоение из $\Lambda$ в некоторое $k$-мерное гладкое многообразие $M$ и $\alpha$-гладкое отображение из $M$ в $\mathbb{R}^{n}$. Отображение $\alpha$ индуцирует отображение $\alpha^{*}$ из $C^{\infty}\left(\mathbb{R}^{n}\right)$ в $C^{\infty}(M)$ посредством $\alpha^{*} f=f \circ \alpha$ и отображение $\alpha_{*}$ из $\left(C^{\infty}\right)^{\prime}(M)$ в $\left(C_{0}^{\infty}\right)^{\prime}\left(\mathbb{R}^{n}\right)$ посредством $\alpha_{*} g=g \circ \alpha^{*}$. Пусть $\mu$-гладкая положительная мера в $M$ с компактным носителем. Тогда обобщенная функция $v=\alpha_{*} \mu$ задает распределение Фурье с лагранжевым многообразием $C$, получаемым из $\Lambda$ фиксированием произвольной точки 
в $T^{*} X$. Индуцированное отображение $\alpha_{*}$ переводит меры на $M$ в меры на $\mathbb{R}^{n}$, так что $v$ также является мерой на $\mathbb{R}^{n}$. Чтобы убедиться в том, что $v=\alpha_{*} \mu$ задает распределение Фурье, запишем $v$ в локальных координатах $z$ в $M$ :

$$
\begin{aligned}
v(x) & =(2 \pi)^{-n} \iint e^{i\langle x-y, \xi\rangle} v(y) d y d \xi \\
& =(2 \pi)^{-n} \iint e^{i\langle x-y, \xi\rangle}\left(\alpha_{*} \mu\right)(y) d y d \xi \\
& =(2 \pi)^{-n} \iint \alpha_{y}^{*}\left(e^{i\langle x-y, \xi\rangle}\right)(z) \mu(z) d z d \xi \\
& =(2 \pi)^{-n} \iint e^{i\langle x-\alpha(z), \xi\rangle} \mu(z) d z d \xi .
\end{aligned}
$$

Распределение Фурье $v$ можно взять в качестве кандидата на обобшенную функцию $w$ выше. Можно проверить, что порядок $v$ как распределения Фурье равен $-k / 2$. Для оператора свертки с $v$ справедлива оценка

$$
\|T f\|_{L^{p}}=\|v * f\|_{L^{p}} \leqslant v\left(\mathbb{R}^{n}\right)\|f\|_{L^{p}},
$$

основанная на инвариантности нормыв $L^{p}$ относительно сдвигов. При $p$, стремящемся к единице, получаем непрерьвность операторов порядка $-k / 2$ в пространствах $L^{p}$, что соответствует теореме 2.9 .

5.3. Применение к интегральным операторам Фурье. Введем следуюшее определение.

ОпРеДЕЛЕниЕ 5.3. Интегральньй оператор Фурье $T \in I_{\rho}^{\mu}(X, Y ; \Lambda)$ называется аналитическим, если множества $X, Y, \Lambda$ вешественно аналитичны.

Заметим, что мы не требуем аналитичности символа в определении, так как нас интересуют свойства операторов с точностью до сглаживаюшего члена. Кроме того, часто удобно предполагать, что символ $T$ сосредоточен на компактном множестве.

Рассмотрим аналитический интегральньй оператор Фурье $T \in I_{\rho}^{\mu}(X, Y ; \Lambda)$ с $1 / 2 \leqslant$ $\rho \leqslant 1$, коммутируюший со сдвигами. Здесь $X$ и $Y$ - открытые множества в $\mathbb{R}^{n}$. Условие коммутирования со сдвигами означает, что $T$ - оператор свертки с некоторой обобшенной функцией $w$. Теория таких операторов как множителей Фурье хорошо известна, но их свойства непрерьвности в $L^{p}$ требуют изучения (см. [38], [59] и предыдущий раздел 5.2). Для случая $\rho=1 / 2$ см. [18]. Обобщенная функция $w$-распределение Фурье, соответствуюшее лагранжеву многообразию $\Lambda(w) \subset T^{*}\left(\mathbb{R}^{n}\right)$, получаемому фиксированием любой точки из $T^{*} Y$. По теореме 2.3 существуют фазовая функция $\phi$ и производяшая функция $H$, для которых $\Lambda(w)$ локально представляется как $\Lambda_{\phi} \mathrm{c}$ положительно однородной по $\xi$ функцией

$$
\phi(z, \xi)=\langle z, \xi\rangle-H(\xi),
$$

где

$$
\Lambda_{\phi}=\{(\nabla H(\xi), \xi)\} .
$$


Распределение Фурье $w$ принимает вид

$$
w(z)=\int e^{i \phi(z, \xi)} a(z, \xi) d \xi
$$

где $a \in S_{\rho}^{\mu}$. Оператор $T$ можно записать в виде

$$
T u(x)=u * w(x)=\iint e^{i \Phi(x, y, \xi)} a(x-y, \xi) u(y) d \xi d y
$$

с фазовой функцией $\Phi(x, y, \xi)=\langle x-y, \xi\rangle-H(\xi)$. Мы предполагаем, что

$$
\left.\operatorname{rank} d \pi_{X \times Y}\right|_{\Lambda} \leqslant n+k
$$

для некоторого $0 \leqslant k \leqslant n-1$ и ранг $n+k$ достигается хотя бы в одной точке. Условие (3) теоремы 2.18 принимает вид

$$
\operatorname{rank} D^{2} H(\xi) \leqslant k
$$

для всех $\xi \in \Xi$. По теореме 5.1 функция $H$ допускает голоморфное продолжение, которое мы также будем обозначать через $H$. Благодаря однородности $H$ можно выделить коническую переменную $\tau$; запишем частотную переменную $\xi$ как $\xi=(\theta, \tau) \in$ $\mathbb{C}^{n-1} \times \mathbb{C}$. Для $\zeta \in \mathbb{C}^{n-1}$ определим $F(\zeta)=H(\zeta, 1)$. Используя однородность $H$, имеем $H(\theta, \tau)=\tau F(\theta / \tau)$ и

$$
\begin{aligned}
\nabla_{\theta} H(\theta, \tau) & =\nabla F(\theta / \tau), \\
\partial_{\tau} H(\theta, \tau) & =-\langle\nabla F(\theta / \tau), \theta / \tau\rangle+F(\theta / \tau), \\
D_{\theta \theta}^{2} H(\theta, \tau) & =D^{2} F(\theta / \tau) / \tau, \\
\partial_{\tau} \nabla_{\theta} H(\theta, \tau) & =-\left\langle D^{2} F(\theta / \tau), \theta / \tau\right\rangle / \tau, \\
\partial_{\tau}^{2} H(\theta, \tau) & =(\theta / \tau)^{T} D^{2} F(\theta / \tau)(\theta / \tau) / \tau .
\end{aligned}
$$

При $\zeta=\theta / \tau$ получаем

$$
D^{2} H(\theta, \tau)=\frac{1}{\tau}\left(\begin{array}{cc}
D^{2} F(\zeta) & -D^{2} F(\zeta) \zeta \\
-\zeta^{T} D^{2} F(\zeta) & \zeta^{T} D^{2} F(\zeta) \zeta
\end{array}\right)
$$

Таким образом, $\operatorname{rank} D^{2} H(\theta, \tau)=\operatorname{rank} D^{2} F(\theta / \tau)$ и условия (5.4) и (5.5) эквивалентны

$$
\operatorname{rank} D^{2} F(\zeta) \leqslant k .
$$

Отображение $F$ голоморфно в некотором открытом подмножестве $\Omega$ в $\mathbb{C}^{n-1}$. Ядра $D^{2} F$ получаются как дополнения в ядрах $D^{2} H$ к коническому направлению. Из теоремы 5.1 следует 
TеОрема 5.4. Пусть $X$ - открытое подмножсество $\mathbb{R}^{n}$, и пусть $\Lambda$ - вещественное аналитическое коническое лагранжево подмногообразие в $T^{*} X \backslash 0$ с производящей функцией $H$, т.е. локально $\Lambda$ имеет вид (5.2), (5.3). Пусть $k \leqslant n-2$ удовлетворяет неравенству

$$
\operatorname{rank} D^{2} H \leqslant k
$$

В окрестности точек $\xi_{n}=1$ зададим $F$ с помощью

$$
F(\zeta)=H(\zeta, 1)
$$

Функиия $F$ вещественно аналитична и ее голоморфное продолэсение обозначим также через $F: \Omega \rightarrow \mathbb{C}$, где $\Omega$ - открытое множсество в $\mathbb{C}^{n-1}$. Тогда отображсение

$$
\nabla F: \Omega \subset \mathbb{C}^{n-1} \rightarrow \mathbb{C}^{n-1}
$$

удовлетворяет условиям (А1)-(А3) введения.

ЗАмЕчАнИЕ 5.5. Напомним, что условие $k \leqslant n-2$ в теореме 5.4 не ограничивает обшности, так как при $k=n-1$ слои функции $\nabla F$ нулевой размерности и условие гладкой факторизации тривиально.

Установив связь с разделом 3 , мы можем использовать его результаты.

ТЕОРема 5.6. Пусть $T \in I_{\rho}^{\mu}(X, Y ; \Lambda)$ - аналитический интегральный оператор Фурье, коммутирующий со сдвигами. Пусть $\mu \leqslant-(k+(n-k)(1-\rho))|1 / p-1 / 2|$, $1<p<\infty, 1 / 2 \leqslant \rho \leqslant 1$. Пусть $\Lambda$ удовлетворяет неравенству

$$
\left.\operatorname{rank} d \pi_{X \times Y}\right|_{\Lambda} \leqslant n+k, \quad 0 \leqslant k \leqslant 2 .
$$

Тогда $T$ непрерывен из $L_{\mathrm{comp}}^{p}(Y)$ в $L_{\mathrm{loc}}^{p}(X)$.

Теорема следует из теорем 5.4, 3.10 и 2.10 .

Tеорема 5.7. Пусть $X, Y$ - открытые множества в $\mathbb{R}^{n}$ с $n \leqslant 4$, и пусть $T \in$ $I_{\rho}^{\mu}(X, Y ; \Lambda)$ - аналитический интегральный оператор Фурье, коммутирующий со сдвигами. Пусть $\mu \leqslant-(k+(n-k)(1-\rho))|1 / p-1 / 2|, 1<p<\infty u 1 / 2 \leqslant \rho \leqslant 1$. Пусть $0 \leqslant k \leqslant 3$ удовлетворяет неравенству

$$
\left.\operatorname{rank} d \pi_{X \times Y}\right|_{\Lambda} \leqslant n+k \text {. }
$$

Тогда T непрерьвен из $L_{\mathrm{comp}}^{p}(Y)$ в $L_{\mathrm{loc}}^{p}(X)$.

Для $k=n-1$ и $k=0$ утверждение следует из теоремы 2.10. Для $k=1$ оно следует из теорем 5.4 и 5.6. Последний случай с $n=4, k=2$ следует из теорем $5.4,3.10$ и 2.10 .

ЗАмЕчАниЕ 5.8. Основная идея двух предыдущих теорем заключается в том, что если $X, Y$ аналитичны и аналитическое коническое лагранжево многообразие $\Lambda \subset T^{*} X \backslash 0 \times T^{*} Y \backslash 0$ соответствует коммутируюшему со сдвигами оператору с $\left.\operatorname{rank} d \pi_{X \times Y}\right|_{\Lambda} \leqslant 2$, то условие гладкой факторизации выполнено.

ЗАмЕчАниЕ 5.9. Пусть оператор $T$ удовлетворяет условиям теорем 5.6 и 5.7. Тогда $T$ непрерьвен из $H^{1}$ в $L^{1}$ при $\mu \leqslant-(k+(n-k)(1-\rho)) / 2$, как следует из теоремы 2.11. 


\section{6. Задача Коши для гиперболических уравнений}

6.1. Оценки решений. Результаты предыдушего раздела можно использовать для улучшения известных оценок в $L^{p}$ для решений с фиксированным временем гиперболической задачи Коши. Для более подробного обсуждения задач Кошши для гиперболических уравнений см. [66], [25], [10], [59], [56]. Рассматриваемый ниже класс содержит операторы с постоянньми коэффициентами. Решения задачи Коши для таких операторов также хорошо известны ([39], [26]). Результаты этого раздела частично содержатся в [48] и [51]. Пусть оператор

$$
P\left(t, \partial_{t}, \partial_{x}\right)=\partial_{t}^{m}+\sum_{j=1}^{m} P_{j}\left(t, \partial_{x}\right) \partial_{t}^{m-j}
$$

строго гиперболический, порядка $m$, на компактном $n$-мерном гладком аналитическом многообразии $X$ c $n \leqslant 4$. Через $p(t, \tau, \xi)$ обозначим главный символ оператора $P$. Строгая гиперболичность означает, что полином $p(t, \tau, \xi)$ имеет $m$ различных вешественных корней по $\tau$. Корни $\tau_{j}(t, \xi)$ - вешественные, однородные по $\xi$ первого порядка и гладкие по $t$. Под задачей Коши мы понимаем уравнение

$$
\begin{cases}P u(t, x)=0, & t \neq 0 \\ \left.\partial_{t}^{j} u\right|_{t=0}=f_{j}(x), & 0 \leqslant j \leqslant m-1\end{cases}
$$

Главньй символ $p$ (или $\sigma_{P}$ ) оператора $P$ допускает представление

$$
p(t, \tau, \xi)=\prod_{j=1}^{m}\left(\tau-\tau_{j}(t, \xi)\right)
$$

Для небольших $t$ решение задачи Коши (6.1) можно получить следуюшим образом. Пусть $\Phi_{j}(t, x, \xi)$ решает уравнение эйконала:

$$
\left\{\begin{array}{l}
\frac{\partial}{\partial t} \Phi_{j}(t, x, \xi)=\tau_{j}\left(t, \nabla_{x} \Phi_{j}\right) \\
\left.\Phi_{j}(t, x, \xi)\right|_{t=0}=\langle x, \xi\rangle
\end{array}\right.
$$

При условии строгой гиперболичности задача Коши (6.1) корректно поставлена и ее решение $u(t, x)$ с точностью до гладкого слагаемого записьвается как конечная сумма эллиптических интегральных операторов Фурье, гладко зависяших от $t$ :

$$
u(t, x)=\sum_{j=1}^{m} \sum_{l=0}^{m-1} \int_{\mathbb{R}^{n}} \int_{\mathbb{R}^{n}} e^{2 \pi i\left(\Phi_{j}(t, x, \xi)-\langle y, \xi\rangle\right)} a_{j l}(t, x, \xi) f_{l}(y) d \xi d y
$$

Канонические отношения локально порождены функциями $\Phi_{j}$ из $(6.2)$, и символы $a_{j l} \in$ $S^{-l}$ находятся из дополнительных уравнений переноса [13], [10]. Выражение (6.3) гладко по $t$. Пусть $f$ - гладкая функция на открытом множестве $U$ в $T^{*} X$. Тогда $d f$ - гладкая 1-форма. Гамильтоновым векторным полем для $f$ назьвается гладкое векторное поле $H_{f}$, удовлетворяюшее тождеству $\sigma\left(v, H_{f}\right)=\langle v, d f\rangle=v(f)$ для всех 
гладких векторных полей $v$. В локальных координатах $(x, \xi) \in U$ поле $H_{f}$ принимает вид

$$
H_{f}=\sum_{j=1}^{n} \frac{\partial f}{\partial \xi_{j}} \frac{\partial}{\partial x_{j}}-\frac{\partial f}{\partial x_{j}} \frac{\partial}{\partial \xi_{j}} .
$$

Для фиксированного значения $t$ каноническое отношение оператора решения из (6.3) имеет вид

$$
C_{t}=\bigcup_{j=1}^{m}\left\{(x, \xi, y, \eta): \chi_{t, j}(y, \eta)=(x, \xi)\right\},
$$

где канонические преобразования $\chi_{t, j}: T^{*} X \backslash 0 \rightarrow T^{*} X \backslash 0$ - потоки из $(y, \eta)$ в момент $t=0$, проходящие через $(x, \xi)$ в момент $t$, вдоль гамильтонова векторного поля

$$
H_{j}=\sum_{j=1}^{n}\left(\frac{\partial \tau_{j}}{\partial \xi_{j}} \frac{\partial}{\partial x_{j}}-\frac{\partial \tau_{j}}{\partial x_{j}} \frac{\partial}{\partial \xi_{j}}\right)=\sum_{j=1}^{n} \frac{\partial \tau_{j}}{\partial \xi_{j}} \frac{\partial}{\partial x_{j}}
$$

в $T^{*} X \backslash 0$. Если рассмотреть $t$ в качестве переменной, то каноническое отношение оператора (6.3) принимает вид

$$
C=\bigcup_{j=1}^{m}\left\{(x, \xi, y, \eta, t, \tau): \tau=\tau_{j}(t, \xi), \chi_{t, j}(y, \eta)=(x, \xi)\right\} .
$$

Используя это представление и асимптотику $D_{\xi \xi}^{2} \Phi_{j}$ по $t$, при дополнительном предположении, что для всех $t$ за исключением некоторого дискретного множества в $\mathbb{R}$ хотя бы один из корней $\tau_{j}$ - эллиптический, можно показать ([53]), что максимальный ранг $D_{\xi \xi}^{2} \Phi_{j}$ равен $2 n-1$. В частности, это условие выполнено для волнового уравнения с переменными коэффищиентами. В обшем, если $k$ - максимальньй ранг матриц $D_{\xi \xi}^{2} \Phi_{j}$, возможно применить результаты раздела 5.3 и получить точные оценки в $L^{p}$ для решений задачи Коши (6.1).

Приведем вначале примеры гиперболических операторов, для которых ранг гессиана фазовой функции оператора решения равен $k<n-1$. Для простоты приведем примеры операторов с постоянными коэффициентами. Как обычно, через $k$ обозначаем максимальный ранг гессианов. Рассмотрим задачу Коши (6.1) в $\mathbb{R}^{1+n}$ с $n \geqslant 3$. Пусть оператор второго порядка $P$ задан с помощью

$$
p\left(\partial_{t}, \partial_{\xi}\right)=\partial_{t}^{2}-\sum_{j=1}^{l+1} \partial_{\xi_{j}}^{2},
$$

плюс члены низших порядков, и $0 \leqslant l \leqslant n-1$. В этом случае корни $\tau_{j}$ имеют вид $\tau_{1}=-\tau_{2}=\left(\sum_{j=1}^{l} \xi_{j}^{2}\right)^{1 / 2}$ и максимальный ранг $k=l$. Порядок $m$ оператора $P$ может быть произвольньм числом $\geqslant 2$, и ранг $k$ может быть любым между нулем и $n-1$. Так, если взять главный символ оператора $P$ равным

$$
\prod_{l=1}^{m / 2}\left(\tau^{2}-\sum_{j=1}^{k} \xi_{j}^{2}-l \xi_{k+1}^{2}\right)
$$

для четных $m$ и при нечетных $m$ умножить его на $\tau-\xi_{1}$, то ранг для такого оператора будет равен $k$. Если заменить $\xi_{1}$ и $\xi_{2}$ на остальные $\xi_{i}$, получим также операторы, включающие производные по всем переменным. 
Теорема 6.1. Пусть $u(t, x)$ решает задачу Kоши $(6.1)$, и пусть $\Phi_{j}(t, x, \xi)$ peшалт уравнение эйконала (6.2). Пусть

$$
k=\max _{x, \xi, j} \operatorname{rank} \frac{\partial^{2}}{\partial \xi^{2}} \Phi_{j}(t, x, \xi)
$$

и $\alpha_{p}=k|1 / p-1 / 2|, 1<p<\infty$. Пусть данные Коши удовлетворяют $f_{j} \in$ $L_{\alpha+\alpha_{p}-j}^{p}(X)$. Тогда $u(t, \cdot) \in L_{\alpha}^{p}(X)$. Более того, если

$$
k=\max _{x, \xi, j, t \in[-T, T]} \operatorname{rank} \frac{\partial^{2}}{\partial \xi^{2}} \Phi_{j}(t, x, \xi)
$$

с фиксированным $0<T<\infty$, то

$$
\|u(t, \cdot)\|_{L_{\alpha}^{p}} \leqslant C_{T} \sum_{j=0}^{m-1}\left\|f_{j}\right\|_{L_{\alpha+\alpha_{p}-j}^{p}}, \quad t \in[-T, T] .
$$

Порядки $\alpha+\alpha_{p}-j$ невозможно улучиить.

ДокАЗАТЕЛЬство. Будем искать решения уравнения эйконала (6.2) в форме

$$
\Phi_{j}(t, x, \xi)=\langle x, \xi\rangle-H_{j}(t, \xi)
$$

Тогда уравнения (6.2) сводятся к задаче Коши для обыкновенных дифференшиальных уравнений:

$$
\frac{\partial H_{j}}{\partial t}(t, \xi)=-\tau_{j}(t, \xi)
$$

с $H_{j}(0, \xi)=0$, где $\xi$ считаем параметром. Ввиду аналитичности $p$ по $\xi$ и строгой гиперболичности, $\tau_{j}$ и $\Phi_{j}$ аналитичны по $\xi$ и гладки по $t$. Так как корни $\tau_{j}$ однородны в $\xi$ с порядком один, получаем, что функции $H_{j}(t, \xi)$ - аналитические и однородные по $\xi$ первого порядка при небольших $t$. Оценки (6.4) следуют из теоремы 5.7. Точность следует из теоремы 7.1.

ЗАмечАниЕ 6.2. Результат теоремы 6.1 можно обобшить на другие размерности $n$ множества $X$. При дополнительном предположении, что $k \leqslant 2$, решение $u(t, \cdot)$ лежит в $L_{\alpha}^{p}$ и выполнены точные оценки (6.4). Доказательство аналогично доказательству теоремы 6.1 и основано на теореме 5.6. Если $X$ не компактно, результат теоремы 6.1 верен в $L_{\text {comp }}^{p}$.

В других функциональных пространствах имеем следующее утверждение.

ТЕОРема 6.3. В условиях теоремы 6.1 справедливы следующие оценки.

(1) Пусть $1<p \leqslant q \leqslant 2$ или $2 \leqslant p \leqslant q<\infty$. Тогда

$$
\|u(t, \cdot)\|_{L_{\alpha}^{q}} \leqslant C_{T} \sum_{j=0}^{m-1}\left\|f_{j}\right\|_{L_{\alpha+\alpha p q}^{p}, j}, \quad t \in[-T, T],
$$

где $\alpha_{p q}=-n / p+k / q+(n-k) / 2$.

(2) В пространствах Липиица $\operatorname{Lip}(\gamma)$ выполнено

$$
\|u(t, \cdot)\|_{\operatorname{Lip}(\alpha)} \leqslant C_{T} \sum_{j=0}^{m-1}\left\|f_{j}\right\|_{\operatorname{Lip}(\alpha+k(n-1) / 2-j)}, \quad t \in[-T, T] .
$$

Приведенные порядки невозможкно улучиить. 
Оценки следуют из соответствующих теорем для интегральных операторов Фурье предыдушего раздела. Точность оценок рассмотрим в следуюшем разделе.

Наилучшими свойствами непрерьвности в $L^{p}$ обладают операторы, для которых порядок $\alpha_{p}$ в теореме 6.1 обрашается в нуль. В этом случае свойства регулярности решений такие же, как и в эллиптических задачах Коши, решение которых дается с помощью псевдодифференциальных операторов. Объяснение этому следующее. В случае $\alpha_{p}=0$ также $k=0$, и из рассуждений следуюшего раздела следует, что операторы решения получаются из псевдодифференциальных операторов композицией с интегральным оператором Фурье, порожденньм некоторой гладкой заменой координат. Забегая вперед, сформулируем теорему.

Теорема 6.4. Пусть $n \in \mathbb{N}, 1<p<\infty u p \neq 2$. Следующие два условия для задачи Коши (6.1) эквивалентны:

(1) Главный символ оператора Р имеет вид

$$
\sigma_{P}(t, \tau, \xi)=\prod_{j=1}^{m}\left(\tau-\tau_{j}(t, \xi)\right),
$$

где $\tau_{j}(t, \xi)$ линейнь по $\xi$.

(2) Для любых данных Коши $f_{j} \in L_{m-j}^{p}$ решение $u(t, \cdot)$ задачи (6.1) принадлежит $L_{m}^{p}$.

B обоих случаях существуют псевдодифференциальные операторы $Q_{j l}, S_{j l} \in$ $\Psi^{-l}(Y)$ и диффеоморфизмы $\sigma_{j}: X \rightarrow Y$ такие, что решение задачи Коши (6.1) имеет вид

$$
u=\sum_{l=0}^{m-1} \sum_{j=1}^{m}\left(\sigma_{j}^{*} \circ Q_{j l}\right) f_{l}=\sum_{l=0}^{m-1} \sum_{j=1}^{m}\left(S_{j l} \circ \sigma_{j}^{*}\right) f_{l},
$$

2де $\sigma_{j}^{*}$ индуцировань отображсениями $\sigma_{j}$. Оценки в пространствах Соболева (6.4) справедливы с $\alpha_{p}=0$.

При $p=2$ условие (2) вьполнено для операторов $P$ произвольного вида, поэтому необходимо исключить случай $p=2$ из формулировки теоремы. Доказательство приведем в разделе 7 .

6.2. Уравнение Монжа-Ампера. Производяшая функция $\phi$ удовлетворяет параметрическому уравнению Монжа-Ампера:

$$
\operatorname{det} \frac{\partial^{2} \phi}{\partial \xi^{2}}(y, \xi)=0
$$

для всех $(y, \xi) \in Y \times \Xi$. Рассмотрим инвариантную версию уравнения (6.10):

$$
\operatorname{det} \frac{\partial^{2} \phi}{\partial \xi^{2}}(\xi)=0, \quad \forall \xi \in \Omega,
$$


соответствуюшую операторам, коммутируюшим со сдвигами. Такие уравнения носят название простейших уравнений Монжса-Ампера, см. [28; 8.2]. Заметим, что если $\phi$ выпукла, то единственное решение уравнения (6.11) должно быть нулевым. В общем, задача

$$
\operatorname{det} \frac{\partial^{2} \phi}{\partial \xi^{2}}(\xi)=f
$$

почти всюду в шаре $B=\left\{\xi \in \mathbb{R}^{n}:|\xi|<1\right\}, n \geqslant 2$, с нулевыми граничными условиями на $\partial B$ и неотрицательной функцией $f \in C^{2}(B)$ имеет единственное решение в $B$ в классе вьпуклых функций. Решение $\phi$ принадлежит $C^{3+\alpha}(\bar{B})$ для всех $\alpha \in(0,1)$, если $f>0$ на $\bar{B}$ (см. [28; 8.2.2]). В нашем случае $f=0$, и пусть $\phi$ решает уравнение (6.11). Выпуклость нам не требуется. Из теорем 3.9 и 3.10 вытекает

СлЕДСТВИЕ 6.5. Пусть $\Omega$ - открытое множество в $\mathbb{R}^{n}\left(\right.$ или $\left.\mathbb{C}^{n}\right)$, и пусть $\phi(\xi)$ - аналитическое решение уравнения Монжа-Ампера (6.11) в $\Omega$ такое, что уровни $\nabla \phi(\xi)$ аффинны для всех $\xi$ смлаксимальнылм рангом $\operatorname{rank} \frac{\partial^{2} \phi}{\partial \xi^{2}}(\xi) \leqslant 2$. Тогда слоение уровнями $\nabla \phi(\xi)$ допускает аналитическое расширение на $\Omega$.

\section{7. Точность оценок}

В этом разделе мы обсудим точность порядков непрерьвных интегральных операторов Фурье для различных рангов проекций. Пусть $X$ и $Y$-гладкие паракомпактные $n$-мерные многообразия, и возьмем $T \in I_{\rho}^{\mu}(X, Y ; \Lambda)$ с каноническим отношением $\Lambda$. В дальнейшем мы сосредоточимся на сушественно однородном случае $(\rho=1)$. Однако, для $1 / 2 \leqslant \rho<1$ точность порядка $\mu=-(n-\rho)|1 / p-1 / 2|$ для непрерьвности в $L^{p}$ можно продемонстрировать на следуюшем примере. Пусть $\Sigma$ - многообразие, описываемое множеством $\left(x^{\prime}, x^{\prime \prime}, y^{\prime}, y^{\prime \prime}\right)$ с $x^{\prime}=y^{\prime}$ и $\left|x^{\prime \prime}-y^{\prime \prime}\right|=1$. Возьмем в качестве $\Lambda^{\prime}$ конормальное расслоение $N^{*} \Sigma$. Пусть $\Psi(\xi)$ - гладкая функция, однородная нулевого порядка для больших $\xi$, сосредоточенная в усеченном конусе

$$
\left\{\xi=\left(\xi^{\prime}, \xi^{\prime \prime}\right) \in \mathbb{R}^{k+1} \times \mathbb{R}^{n-k-1}:\left|\xi^{\prime}\right| \geqslant 1,\left|\xi^{\prime \prime}\right| / 2 \leqslant\left|\xi^{\prime}\right| \leqslant 2\left|\xi^{\prime \prime}\right|\right\}
$$

и равная единице в открытом усеченном подконусе. Рассмотрим оператор $T$ свертки с ядром

$$
K(x, y)=\int \Psi(\xi) e^{i|\xi|^{1-\rho}}|\xi|^{\mu} e^{i\left[\langle x-y, \xi\rangle+\left|\xi^{\prime}\right|\right]} d \xi
$$

Несложно проверить, что $T \in I_{\rho}^{\mu}\left(\mathbb{R}^{n}, \mathbb{R}^{n} ; \Lambda\right)$. Пусть $\widehat{g}_{\sigma}(\xi)=\left(1+|\xi|^{2}\right)^{-\sigma / 2}$. Тогда $g_{\sigma} \in L^{p}$ при $\sigma>n(1-1 / p)$. Действуя оператором $T$ на $g_{\sigma}$ и применяя метод стационарной фазы в полярных координатах для $\xi^{\prime}$, можно показать, что $T g_{\sigma} \notin L_{\mathrm{loc}}^{p}$ при $\sigma$, стремящемся к $n(1-1 / p)$, и $\mu>-(n-\rho)|1 / p-1 / 2|$. Асимптотические приближения для таких операторов были рассмотрены в [67], и сами операторы использованы в [53] для аргументации точности порядка $\mu$ в теореме 2.10 .

7.1. Существенно однородный случай. В существенно однородном случае с $\rho=1$ можно показать точность порядка $\mu=-k|1 / p-1 / 2|$ для произвольных непрерывных в $L^{p}$ эллиптических операторов, удовлетворяюших условию

$$
\left.\operatorname{rank} d \pi_{X \times Y}\right|_{\Lambda} \leqslant n+k .
$$


Для проверки отсутствия непрерывности в $L^{p}$ оказывается достаточным подействовать оператором $T$ на функции $f$ с точечными особенностями в $L^{p}$. В этом случае особенности $T f$ могут возникать только в направлениях, трансверсальных некоторому фиксированному $k$-мерному многообразию в $X$.

ТЕОРема 7.1. Пусть каноническое отношение $\Lambda$ - локальный график, удовлетворяющий условию $\left.\operatorname{rank} d \pi_{X \times Y}\right|_{\Lambda} \leqslant n+k c 0 \leqslant k \leqslant n-1$, дде ранг $n+k$ достигается хотя бы в одной точке. Тогда әллиптические операторы $T \in I^{\mu}(X, Y ; \Lambda)$ не непрерывны из $L_{\mathrm{comp}}^{p}(Y)$ в $L_{\mathrm{loc}}^{p}(X)$, если $\mu>-k|1 / p-1 / 2| u 1<p<\infty$.

Сразу отметим, что по теореме о выборе фазовой функции достаточно рассмотреть операторы $T$ в $\mathbb{R}^{n}$ с ядром, локально заданным формулой

$$
K(x, y)=\int_{\mathbb{R}^{n}} e^{i[\langle x, \xi\rangle-\phi(y, \xi)]} b(x, y, \xi) d \xi,
$$

с символом $b \in S^{\mu}$, сосредоточенным на компактном множестве по $x$ и $y$. Условие локального графика означает, что фазовая функция $\phi$ удовлетворяет

$$
\operatorname{det} \phi_{y \xi}^{\prime \prime} \neq 0
$$

на носителе $b$. Локально $\Lambda$ имеет вид $\left\{\left(\nabla_{\xi} \phi, \xi, y, \nabla_{y} \phi\right)\right\}$. Можно предположить, что $1<p \leqslant 2$. Для $2<p$ достаточно рассмотреть сопряженный оператор $T^{*}$, и утверждение следует из результата для сопряженного индекса $p^{\prime}=p /(p-1)<2$.

Множество $\Lambda_{0}=\left\{\lambda \in \Lambda:\left.\operatorname{rank} d \pi_{X \times Y}\right|_{\Lambda}(\lambda)=n+k\right\}$ не пусто и открыто в $\Lambda$. Возьмем $\lambda_{0}=\left(x_{0}, \xi_{0}, y_{0}, \eta_{0}\right) \in \Lambda_{0}$. Рассмотрим семейство функций $f_{s}(y)=$ $\left((I-\Delta)^{-s / 2} \delta_{y_{0}}\right)(y)$ при некотором фиксированном значении $y_{0} \in Y$. Пусть $K_{-s}-$ интегральное ядро для $(I-\Delta)^{-s / 2}$. Имеем

$$
f_{s}(y)=\int K_{-s}(y, z) \delta_{y_{0}}(z) d z=K_{-s}\left(y, y_{0}\right) .
$$

Используя стандартные оценки для ядра Шварца псевдодифференциального оператоpa ([55], [10], [59]), получаем $\left|K_{-s}\left(y, y_{0}\right)\right| \leqslant C\left|y-y_{0}\right|^{-n+s}$ в некоторой локальной системе координат. Это означает, что $f_{s} \in L_{\mathrm{loc}}^{p}$ тогда и только тогда, когда $s>n(1-1 / p)$.

Пусть $\Sigma=\pi_{X \times Y}(\Lambda \cap U)$, где $U \subset \Lambda_{0}$ - некоторая окрестность $\lambda_{0}$. Так как ранг $\pi_{X \times Y}$ постоянен в $U$, множество $\Sigma \subset X \times Y$ - гладкое $k$-мерное многообразие, заданное уравнениями $h_{j}(x, y)=0,1 \leqslant j \leqslant n-k$, в окрестности $y_{0}$. Векторы $\nabla h_{1}, \ldots, \nabla h_{n-k}$ линейно независимы на $\Sigma$. Тогда $\Lambda$ микролокально является конормальным расслоением к $\Sigma$, и фазовая функция оператора $T$ принимает вид

$$
\Phi(x, y, \lambda)=\sum_{j=1}^{n-k} \lambda_{j} h_{j}(x, y) .
$$

Так как композиция с псевдодифференциальным оператором не меняет канонического отношения, получаем, что оператор $T \circ(I-\Delta)^{-s / 2} \in I^{\mu-s}(X, Y ; \Lambda)$. Имеем равенство $T f_{s}(x)=T \circ(I-\Delta)^{-s / 2}\left(\delta_{y_{0}}\right)(x)$, которое в локальных координатах принимает вид

$$
\begin{aligned}
T f_{s}(x) & =\int_{\mathbb{R}^{n}}\left(\int_{\mathbb{R}^{n-k}} e^{i \sum \lambda_{j} h_{j}(x, y)} a(x, \bar{\lambda}) \delta_{y_{0}}(y) d \bar{\lambda}\right) d y \\
& =\int_{\mathbb{R}^{n-k}} e^{i\left(\bar{\lambda}, \bar{h}\left(x, y_{0}\right)\right\rangle} a(x, \bar{\lambda}) d \bar{\lambda} \\
& =(2 \pi)^{n-k}\left(\mathscr{F}^{-1} a\right)\left(x, \bar{h}\left(x, y_{0}\right)\right),
\end{aligned}
$$


где $\bar{\lambda}$ и $\bar{h}$ - векторы с компонентами $\lambda_{j}$ и $h_{j}$ и $\mathscr{F}^{-1}$ - обратное преобразование $\Phi$ урье. Символ $a \in S^{\mu-s+k / 2}\left(\mathbb{R}^{n-k}\right)$ получается из символа оператора $T \circ(I-\Delta)^{-s / 2}$ применением метода стационарной фазы, в котором мы избавляемся от $k$ переменных. Обратное преобразование Фурье символа $a$ по второй переменной имеет вид

$$
(2 \pi)^{n-k}\left(\mathscr{F}^{-1} a\right)(x, \zeta)=\int_{\mathbb{R}^{n-k}} e^{i\langle\lambda, \zeta\rangle} a(x, \lambda) \widehat{\delta}_{0}(\lambda) d \lambda=P \delta_{0}(\zeta)=K(\zeta, 0),
$$

где $P \in \Psi^{m}\left(\mathbb{R}^{n-k}\right)$ - псевдодифференциальный оператор порядка $m=n-s+k / 2$ с символом $a(x, \lambda) \in S^{m}$ и $K$ - интегральное ядро оператора $P$. Функция $K(\zeta, 0)$ эквивалентна $|\zeta|^{-(n-k)-m}$. Для множества $\Sigma_{y_{0}}=\left\{x:\left(x, y_{0}\right) \in \Sigma\right\}$ имеем $\operatorname{dist}\left(x, \Sigma_{y_{0}}\right) \approx$ $\left|\bar{h}\left(x, y_{0}\right)\right|$, и поэтому

$$
(2 \pi)^{n-k}\left(F^{-1} a\right)\left(x, \bar{h}\left(x, y_{0}\right)\right) \sim\left|\operatorname{dist}\left(x, \Sigma_{y_{0}}\right)\right|^{-(n-k)-(\mu-s+k / 2)}
$$

локально равномерно по $x$. Из формулы (7.4) видно, что функция $T f_{s}$ гладкая на $\Sigma_{y_{0}}$, поэтому $T f_{s} \notin L_{\mathrm{loc}}^{p}\left(\mathbb{R}^{n}\right)$ тогда и только тогда, когда

$$
p(n-k+\mu-s+k / 2) \geqslant n-k .
$$

Для $s$ это означает, что $s \leqslant \mu+(n-k)(1-1 / p)+k / 2$. Таким образом, для того чтобы $f_{s} \in L_{\mathrm{loc}}^{p}$ и $T f_{s} \notin L_{\mathrm{loc}}^{p}$, достаточно, чтобы вьполнялось неравенство

$$
n(1-1 / p)<\mu+(n-k)(1-1 / p)+k / 2,
$$

что равносильно $\mu>-k|1 / p-1 / 2|$.

Для $L^{p}-L^{q}$ непрерывности имеем:

СлЕДСТВИЕ 7.2. Пусть Т и $p, q<\infty$. Тогда $T$ не непрерывен из $L_{\mathrm{comp}}^{p}(Y)$ в $L_{\mathrm{loc}}^{q}(X) n p и \mu>n(1 / q-1 / p)-$ $k(1 / q-1 / 2)$.

Для доказательства можно применить точно такой же аргумент, как и при доказательстве теоремы 7.1 , за исключением того, что теперь не нужно предполагать, что $p \leqslant 2$, и неравенство (7.5) заменяется на

$$
q(n-k+\mu-s+k / 2) \geqslant n-k .
$$

ЗАМЕчАнИЕ 7.3 . При $k=n-1$ и $1<p \leqslant q \leqslant 2$ утверждение следствия 7.2 дополняет теорему $2.12,(2)$.

По двойственности получаем утверждение, аналогичное утверждению теоремы 7.6, для индексов $2<p \leqslant q<\infty$.

ЗАМЕчАнИЕ 7.4. Оператор $T$ в теореме 7.1 не непрерьвен как линейньй оператор в пространствах Соболева $L_{\alpha}^{p} \rightarrow L_{\alpha-k|1 / p-1 / 2|-\mu}^{p}, 1<p<\infty$. 
7.2. Представление непрерывных операторов небольших отрицательных порядков. Хорошо известно, что псевдодифференциальные операторы нулевого порядка непрерывны в $L^{p}$ при $1<p<\infty$ (см. раздел 2.3). Сейчас мы покажем, что все непрерывные в $L^{p}$ эллиптические интегральные операторы Фурье можно получить из псевдодифференциальных операторов композицией с операторами, индуцированными гладкой заменой переменных. Гладкое отображение $\sigma: X \rightarrow Y$ индуцирует отображение $\sigma^{*}: C^{\infty}(Y) \rightarrow C^{\infty}(X)$, заданное формулой $\left(\sigma^{*} f\right)(x)=f(\sigma(x))$. Нетрудно показать, что $\sigma^{*}$ - интегральный оператор Фурье с фазовой функцией $\langle\sigma(x)-y, \eta\rangle$. Каноническое соотношение $\sigma^{*}$ получается как график преобразования $\widetilde{\sigma}: T^{*} X \backslash 0 \rightarrow T^{*} Y \backslash 0$ c $\widetilde{\sigma}(x, \xi)=\left(\sigma(x),-\left({ }^{t} D \sigma_{x}\right)^{-1}(\xi)\right)$.

TeOPEMA 7.5. Пусть $1<p<\infty, p \neq 2, u 0 \geqslant \mu>-|1 / p-1 / 2|$. Пусть $\Lambda$ удовлетворяет условию локального графика. Тогда әллиптический оператор $T \in I^{\mu}(X, Y ; \Lambda)$ непрерьвен из $L_{\mathrm{comp}}^{p}(Y)$ в $L_{\mathrm{loc}}^{p}(X)$ тогда и только тогда, когда существуют псевдодифференциальные операторы $P \in \Psi^{\mu}(X), Q \in \Psi^{\mu}(Y)$ такие, что $T=P \circ \sigma^{*}=\sigma^{*} \circ Q$, әде отображение $\sigma^{*}$ индуцировано гладкой заменой координат из $X$ в $Y$.

ДокАЗАтельство. Оператор $\sigma^{*}$ непрерьвен в $L^{p}$. Псевдодифференциальные операторы $P$ и $Q$ порядка $\mu \leqslant 0$ непрерьвны в $L^{p}$, поэтому $T$ также непрерывен в $L^{p}$. Обратно, пусть для $k$ выполнено $n+k=\left.\max _{\lambda \in \Lambda} \operatorname{rank} d \pi_{X \times Y}\right|_{\Lambda}(\lambda)$. Тогда $n-k-$ максимальная размерность множества $\Sigma=\pi_{X \times Y}(\Lambda) \subset X \times Y$. По теореме 7.1 и непрерывности $T$ в $L^{p}$ необходимо, чтобы $k=0$. Это означает, что $\left.\operatorname{rank} d \pi_{X \times Y}\right|_{\Lambda} \equiv n$ и $\Sigma$ - гладкое $n$-мерное многообразие в $X \times Y$. Ранг $\left.d \pi_{X}\right|_{\Sigma}$ проекции $\pi_{X}: X \times Y \rightarrow X$ должен быть равен $n$, так как $\Lambda$ - локальный график. Из сюръективности отображения $\left.d \pi_{X}\right|_{\Sigma}$ и $\operatorname{dim} \Sigma=n$ следует, что $\left.\pi_{X}\right|_{\Sigma}-$ диффеоморфизм и локально $\Sigma$ может быть задана как $\Sigma=\{(x, \sigma(x))\}$, с некоторьм диффеоморфизмом $\sigma: X \rightarrow Y$. Тогда каноническое отношение оператора $\sigma^{*}-$ конормальное расслоение к $\Sigma$, т.е. равно $\Lambda$. Поэтому оператор $Q$ в $T=\sigma_{+}^{*} \circ Q$ должен быть псевдодифференциальным, так как его каноническое отношение - конормальное расслоение к диагонали в $X \times Y$. Из аналогичного рассуждения в $Y$ следует формула для $P$ с тем же отображением $\sigma$, так как каноническое отношение $\sigma^{*}$ равно $\Lambda$.

TEOPEMA 7.6. Пусть $1<p \leqslant q<2 u-n(1 / p-1 / q) \geqslant \mu>-(1 / q-1 / 2)-$ $n(1 / p-1 / q)$. Пусть $T \in I^{\mu}(X, Y ; \Lambda)$ - әллиптический оператор и $\Lambda$ - локальный график. Тогда T непрерывен из $L_{\mathrm{comp}}^{p}(Y)$ в $L_{\mathrm{loc}}^{q}(X)$ тогда и только тогда, когда существуют псевдодифференииальнье операторы $P \in \Psi^{\mu}(X), Q \in \Psi^{\mu}(Y)$ такие, что $T=P \circ \sigma^{*}=\sigma^{*} \circ Q$, где $\sigma^{*}$ индуцировано некоторым диффеоморфизмом $\sigma: X \rightarrow Y$.

Непрерьвность оператора $T$ следует из непрерьвности из $L^{p}$ в $L^{q}$ псевдодифференциальных операторов порядка $-n(1 / p-1 / q)$ (утверждение 2.16$)$.

Заметим также, что следствие $7.2 \mathrm{c} k=n$ влечет точность порядков утверждения 2.16 для эллиптических операторов $P$. Наконец, докажем теорему 6.4 .

ДОКАЗАТЕЛЬСТВО ТЕОРЕМЫ 6.4. Предположим, что однородная часть наивысшего порядка оператора $P$ имеет вид (6.8). Дифференцируя уравнение (6.5) по $\xi$ дважды, получим $\partial_{\xi \xi}^{2} \partial_{t} H_{j}(t, \xi)=0$. Следовательно, $\partial_{\xi \xi}^{2} H_{j}(t, \xi)=\partial_{\xi \xi}^{2} H_{j}(0, \xi)=0$ и $H_{j}$ линейны по $\xi$. Ранг $k$ в теореме 6.1 тогда равен нулю, из чего следует вторая часть теоре- 
мы, так как псевдодифференциальные операторы порядка $-j$ непрерывны из $\left(L_{\alpha}^{p}\right)$ comp В $\left(L_{\alpha+j}^{p}\right)_{\text {loc }}$.

Обратно, пусть $P$ удовлетворяет условию второй части теоремы. Если $T_{l}-$ оператор порядка $-l$ в $(6.3)$, то применяя теорему 7.5 к оператору $T=T_{l} \circ(I-\Delta)^{l / 2}$, получаем формулу (6.9). Кроме того, $k=0$, и поэтому $\partial_{\xi \xi}^{2} \tau_{j}(t, \xi)=-\partial_{t} \partial_{\xi \xi}^{2} H_{j}(t, \xi)=0$. Таким образом, $\tau_{j}$ должны быть полиномами порядка $\leqslant 1$ по $\xi$. Так как они однородны с порядком один, то они также линейны. Оценки в пространствах Соболева следуют из непрерьвности псевдодифференциальных операторов порядка $-j$ из $\left(L_{\alpha}^{p}\right)$ сотр В $\left(L_{\alpha+j}^{p}\right)_{\text {loc }}$.

\section{СПИСОК ЛИТЕРАТУРЫ}

[1] Арнольд В.И. Особенности каустик и волновых фронтов. М.: Фазис, 1996.

[2] Арнольд В.И., Варченко А. Н., Гусейн-Заде С. М. Особенности дифференцируемых отображений. М.: Наука, 1982.

[3] Beals R. M. $L^{p}$ boundedness of Fourier integrals operators // Mem. Amer. Math. Soc. 1982. V. 264. P. 1-57.

[4] Bennett C., Sharpley R. Interpolation of Operators. Boston: Academic Press, 1988. (Pure Appl. Math. V. 129.)

[5] Björk J. E. Rings of Differential Operators. Amsterdam: North-Holland, 1979.

[6] Chow W. L. On compact analytic varieties // Amer. J. Math. 1955. V. 71. P. 893-914.

[7] Colin de Verdière Y., Frisch M. Régularité lipschitzienne et solutions de l'équation des ondes sur une variété riemannienne compacte // Ann. Sci. École Norm. Sup. (4). 1976. V. 9. P. 539-565.

[8] Duistermaat J. J. Fourier Integral Operators. New York: Courant Inst. of Math. Sci., New York University, 1973.

[9] Duistermaat J. J. Classical Aspects of Partial Differential Equations. Utrecht: Rijksuniversiteit Utrecht, 1980. (Comm. Math. Inst. V. 13.)

[10] Duistermaat J. J. Fourier Integral Operators. Boston: Birkhäuser, 1996.

[11] Duren P. L. Theory of $H^{p}$ Spaces. New York: Academic Press, 1970. (Pure Appl. Math. V. 38.)

[12] Егоров Ю. В. Микролокальный анализ // Итоги науки и техн. Совр. пробл. матем. Фунд. направления. Т. 33. М.: ВИНИТИ, 1988.

[13] Егоров Ю. В., Шубин М. А. Линейные дифференциальные уравнения с частными производными. Элементы современной теории // Итоги науки и техн. Совр. пробл. матем. Фунд. направления. Т. 31. М.: ВИНИТИ, 1988.

[14] Эскин Г. И. Вырожденные эллиптические псевдодифференциальные операторы принципиального типа // Матем. сб. 1970. Т. 82. С. 585-628.

[15] Fefferman C. $L^{p}$ bounds for pseudo-differential operators // Israel J. Math. 1973. V. 14. P. 413-417.

[16] Fefferman C., Stein E. M. $H^{p}$ spaces of several variables // Acta Math. 1972. V. 129. P. 137-193.

[17] Greenleaf A., Uhlmann G. Non-local inversion formulas for the X-ray transform // Duke Math. J. 1989. V. 58. P. 205-240.

[18] Greenleaf A., Uhlmann G. Estimates for singular Radon transforms and pseudo-differential operators with singular symbols // J. Funct. Anal. 1990. V. 89. P. 202-232.

[19] Guillemin V. Cosmology in (2+1) Dimensions, Cyclic Models, and Deformations of $M_{2,1}$. Princeton: Princeton Univ. Press, 1989.

[20] Guillemin V., Uhlmann G. Oscillatory integrals with singular symbols // Duke Math. J. 1981. V. 48. P. 251-267.

[21] Hardy G. H., Littlewood J. E. Some properties of fractional integrals // Math. Z. 1928. V. 27. P. 565-606. 
[22] Хартшорн Р. Алгебраическая геометрия. М.: Мир, 1981.

[23] Hörmander L. Fourier integral operators, I // Acta Math. 1971. V. 127. P. 79-183.

[24] Хёрмандер Л. Анализ линейных дифференциальных операторов с частными производными. Т. 3-4. М.: Мир, 1987-1988.

[25] Иврий В.Я. Линейные гиперболические уравнения // Итоги науки и техн. Совр. пробл. матем. Фунд. направления. Т. 33. М.: ВИНИТИ, 1988.

[26] Комеч А. И. Линейные уравнения в частных производных с постоянными коэффициентами // Итоги науки и техн. Совр. пробл. матем. Фунд. направления. Т. 31. М.: ВИНИТИ, 1988.

[27] Кондратьев В. А., Ландис Е. М. Качественная теория линейных дифференциальных уравнений в частных производных второго порядка // Итоги науки и техн. Совр. пробл. матем. Фунд. направления. Т. 32. М.: ВИНИТИ, 1988.

[28] Крылов Н. В. Нелинейные эллиптические и параболические уравнения второго порядка. M.: Наука, 1985.

[29] Łojasiewicz S. Introduction to Complex Analytic Geometry. Basel: Birkhäuser, 1991.

[30] Laptev A., Safarov Yu., Vassiliev D. On global representation of Lagrangian distributions and solutions of hyperbolic equations // Comm. Pure Appl. Math. 1994. V. 47. P. 1411-1456.

[31] Littman W. $L^{p}-L^{q}$-estimates for singular integral operators arising from hyperbolic equations // Proc. Sympos. Pure Math. 1973. V. 23. P. 479-481.

[32] Маслов В.П., Федорюк М. В. Квазиклассическое приближение для уравнений квантовой механики. М.: Наука, 1976.

[33] Melin A., Sjöstrand J. Fourier integral operators with complex-valued phase functions // Lecture Notes in Math. 1975. V. 459. P. 120-223.

[34] Melin A., Sjöstrand J. Fourier integral operators with complex phase functions and parametrix for an interior boundary value problem // Comm. Partial Differential Equations. 1976. V. 1. P. 313-400.

[35] Михлин С. Г. Многомерные сингулярные интегралы и интегральные уравнения. М.: Физматгиз, 1962

[36] Miyachi A. On some estimates for the wave operator in $L^{p}$ and $H^{p} / /$ J. Fac. Sci. Univ. Tokyo Sect. IA Math. 1980. V. 27. P. 331-354.

[37] Nagel A., Stein E. M. Lectures on Pseudo-Differential Operators: Regularity Theorems and Applications to Non-Elliptic Problems. Princeton: Princeton Univ. Press, 1979. (Math. Notes. V. 24.)

[38] Oberlin D. M. Convolution estimates for some measures on curves // Proc. Amer. Math. Soc. 1987. V. 99. P. 56-60.

[39] Паламодов В. П. Линейные дифференциальные операторы с постоянными коэффициентами. М.: Наука, 1967.

[40] Peral J. $L^{p}$ estimates for the wave equation // J. Funct. Anal. 1980. V. 36. P. 114-145.

[41] Phong D. H. Regularity of Fourier integral operators // Proceedings of the International Congress of Mathematicians (Zürich, 1994).V. II / ed. S.D. Chatterji. Basel: Birkhäuser, 1995. P. 862-874.

[42] Phong D. H. Singular integrals and Fourier integral operators // Essays on Fourier Analysis in Honor of E.M. Stein / ed. C. Fefferman, R. Fefferman, S. Wainger. Princeton: Princeton Univ. Press, 1995. P. 286-320.

[43] Phong D. H., Stein E. M. Radon transforms and torsion // Internat. Math. Res. Notices. 1991. V. 4. P. 49-60.

[44] Phong D. H., Stein E. M. Oscillatory integrals with polynomial phases // Invent. Math. 1992. V. 110. P. 39-62.

[45] Picard E., Simart G. Théorie des fonctions algébriques de deux variables indépendentes. Paris, 1897-1906.

[46] Remmert R. Holomorphe und meromorphe Abbildungen komplexer Räume // Math. Ann. 1957. V. 133. P. 328-370. 
[47] Ruzhansky M. Analytic Fourier integral operators, Monge-Ampère equation and holomorphic factorization // Arch. Mat. 1999. V. 72. P. 68-76.

[48] Ruzhansky M. Holomorphic factorization for the solution operators for hyperbolic equations // Internat. Ser. Numer. Math. 1999. V. 130. P. 803-811.

[49] Ruzhansky M. On the sharpness of Seeger-Sogge-Stein orders // Hokkaido Math. J. 1999. V. 28. P. 357-362.

[50] Ruzhansky M. Singular fibrations with affine fibers (to appear).

[51] Ruzhansky M. Sharp Estimates for a Class of Hyperbolic Partial Differential Operators. Edinburgh: University of Edinburgh, 1999.

[52] Ruzhansky M. On the failure of the factorization condition for the invariant wave fronts // Proc. Amer. Math. Soc. (to appear).

[53] Seeger A., Sogge C. D., Stein E. M. Regularity properties of Fourier integral operators // Ann. of Math. 1991. V. 134. P. 231-251.

[54] Шафаревич И. Р. Основы алгебраической геометрии. М.: Наука, 1988.

[55] Шубин М.А. Псевдодифференциальные операторы и спектральная теория. М.: Наука, 1978.

[56] Sogge C. D. Fourier Integrals in Classical Analysis. Cambridge: Cambridge Univ. Press, 1993.

[57] Стейн И. М. Сингулярные интегралы и дифференциальные свойства функций. М.: Мир, 1973.

[58] Stein E. M. $L^{p}$ boundedness of certain convolution operators // Bull. Amer. Math. Soc. 1971. V. 77. P. 404-405.

[59] Stein E. M. Harmonic Analysis. Princeton: Princeton Univ. Press, 1993.

[60] Stein E. M., Wainger S. Problems in harmonic analysis related to curvature // Bull. Amer. Math. Soc. 1978. V. 84. P. 1239-1295.

[61] Stein E. M., Weiss G. Introduction to Fourier Analysis on Euclidean Spaces. Princeton: Princeton Univ. Press, 1971.

[62] Sugimoto M. On some $L^{p}$-estimates for hyperbolic equations // Ark. Mat. 1992. V. 30. P. $149-162$.

[63] Taylor M. E. Propagation, reflection, and diffraction of singularities of solutions to wave equation // Bull. Amer. Math. Soc. 1978. V. 84. P. 589-611.

[64] Taylor M. E. Fefferman-Phong inequalities in diffraction theory // Proc. Sympos. Pure Math. 1985. V. 43. P. 261-300.

[65] Трибель Х. Теория функциональных пространств. М.: Мир, 1986.

[66] Волевич Л. П., Гиндикин С. Г. Задача Коши // Итоги науки и техн. Совр. пробл. матем. Фунд. направления. Т. 32. М.: ВИНИТИ, 1988.

[67] Wainger S. Special trigonometric series in k-dimensions // Mem. Amer. Math. Soc. 1965. V. 59. P. 1-98.

[68] Зигмунд А. Тригонометрические ряды. Т. 1, 2. М.: Мир, 1965.

Университет Эдинбурга

Поступила в редакцию

09.12 .1999 